\title{
Deformation spaces of Kleinian surface groups are not locally connected
}

\author{
AARON D MAGID
}

\begin{abstract}
For any closed surface $S$ of genus $g \geq 2$, we show that the deformation space $A H(S \times I)$ of marked hyperbolic 3-manifolds homotopy equivalent to $S$ is not locally connected. This proves a conjecture of Bromberg who recently proved that the space of Kleinian punctured torus groups is not locally connected. Playing an essential role in our proof is a new version of the filling theorem that is based on the theory of cone-manifold deformations developed by Hodgson, Kerckhoff and Bromberg.
\end{abstract}

57M50; 30F40

\section{Introduction}

Given a compact, orientable 3-manifold $N$, let $A H(N)$ denote the set of marked hyperbolic 3-manifolds homotopy equivalent to $N$, equipped with the algebraic topology. The interior of $A H(N)$, as a subset of the $\operatorname{PSL}(2, \mathbb{C})$-character variety, is well-understood; however, little is known about the topology of entire deformation space and its dependence on the topology of $N$. We show that there are points where $A H(N)$ fails to be locally connected when $N$ is homotopy equivalent to a closed surface.

The work of Ahlfors [3], Bers [8], Kra [42], Marden [49], Maskit [54], Sullivan [69] and Thurston [71] shows that the components of the interior of $A H(N)$ are in one-toone correspondence with the marked homeomorphism types of compact 3-manifolds homotopy equivalent to $N$. Using the theory of quasiconformal deformations and the measurable Riemann mapping theorem, each of these components can be parameterized by analytic information.

Unfortunately, our understanding of the interior of $A H(N)$ does not extend to the entire space. When the boundary of $N$ is incompressible, Anderson, Canary and McCullough [5] characterized when two components of the interior of $A H(N)$ have intersecting closures. They called this phenomenon bumping. For any genus $g \geq 2$ surface $S$, McMullen [59] showed that the interior of $A H(S \times I)$ self-bumps. This 
means that there is a point $\rho \in A H(S \times I)$ such that whenever $U$ is a sufficiently small neighborhood of $\rho$, the intersection of $U$ and the interior of $A H(S \times I)$ is disconnected. Bromberg and Holt [21] generalized this result by showing that whenever $N$ contains a primitive, essential annulus that is not homotopic into a torus boundary component of $N$ then the interior of $A H(N)$ self-bumps.

Recent work by Agol [2], Calegari and Gabai [24], Brock, Canary and Minsky [14], and many others has led to a classification of hyperbolic manifolds up to isometry. The existence of bumping and self-bumping points shows that the invariants used in this classification do not vary continuously at certain points on the boundary of the deformation space (see also Brock [10]). Thus, further study of the local topology of $A H(N)$ near these points is necessary in order to fully understand these spaces of hyperbolic manifolds.

Bromberg [20] recently showed that the space of Kleinian punctured torus groups is not locally connected. The points where this deformation space fails to be locally connected are self-bumping points, but he also showed that the space is locally connected at other self-bumping points. This indicates that bumping may be considerably more complicated than we previously thought. He also conjectured that $A H(S \times I)$ would fail to be locally connected for any surface $S$, although his arguments in the punctured torus case made essential use of Minsky's [61] classification of punctured torus groups. The results in [61] that Bromberg uses do not generalize to higher genus surfaces.

The following theorem proves Bromberg's conjecture.

Theorem 1.1 Let $S$ be a closed surface of genus $g \geq 2$. Then $A H(S \times I)$ is not locally connected.

Using Bromberg's description of the space of punctured torus groups, one can show that many relative deformation spaces fail to be locally connected (see the author's paper [48]), although Theorem 1.1 provides the first examples of nonrelative deformation spaces that fail to be locally connected. Nonrelative means that no parabolicity condition is specified. See Section 2 for a discussion of pared manifolds and deformation spaces.

The failure of local connectivity of deformation spaces also provides an interesting entry into the Sullivan dictionary [68]. In this analogy, a Kleinian group corresponds to a rational map, limit sets correspond to Julia sets, and deformation spaces such as $A H(S \times I)$ correspond to connectedness loci in parameter spaces of rational maps. It is a major open conjecture that the Mandelbrot set is locally connected and proving this would have many implications for the classification of quadratic polynomials. For example, local connectivity implies that a quadratic map admits no invariant line field 
on its Julia set and hyperbolic maps are dense among quadratic maps; see Douady and Hubbard [30] and McMullen [57; 56]. The corresponding one (complex) dimensional deformation spaces such as the Maskit slice or Bers' slices in the deformation space of punctured torus groups are locally connected [61]. In higher dimensions, the cubic connectedness locus is not locally connected by Lavaurs [45] and Milnor [60], and Theorem 1.1 builds on Bromberg [20, Theorem 4.15] to show that all deformation spaces of Kleinian surface groups are not locally connected.

In our proof that $A H(S \times I)$ fails to be locally connected for any higher-genus surface $S$ we frequently refer to many of the arguments in [20]. In particular, the results in Section 5 rely on and/or generalize the results of [20, Section 3]. However, since some of Minsky's results in [61] do not extend to higher genus surfaces, we make a significant departure from Bromberg's methods in Section 6. In this section, we make use of an improved version of the filling theorem, the key technical result of this paper.

Before stating our version of the filling theorem, we will set up some of the notation. Given a geometrically finite hyperbolic manifold $\widehat{M}$ with a rank-2 cusp, the filling theorem provides sufficient conditions for one to "Dehn-fill" the cusp. That is, if $\widehat{M}$ is homeomorphic to the interior of a compact manifold $\widehat{N}$ with a torus boundary component corresponding to the cusp of $\widehat{M}$, and $N$ is a Dehn-filling of $\widehat{N}$, then the filling theorem provides conditions for one to construct a hyperbolic manifold $M$ homeomorphic to the interior of $N$ with the same conformal boundary as $\widehat{M}$. Assuming the hypotheses of the theorem are satisfied, one obtains a relationship between the metrics on $\widehat{M}$ and $M$.

Suppose $T$ is a rank-2 cusp in $\widehat{M}$ and $\beta$ is the slope in $T$ along which we are filling. Let $L$ be the normalized length of $\beta$ in $T$, and let $A^{2}$ be the reciprocal of the normalized twist of the cusp. Although we relegate the actual definitions of the normalized length and the normalized twist to Section 4, we now describe these quantities with respect to a particular normalization of the cusp (the normalization that we will use throughout Section 5 and Section 6). Suppose the rank-2 cusp $T$ of $\widehat{M}$ is generated by parabolics

$$
\left(\begin{array}{ll}
1 & 2 \\
0 & 1
\end{array}\right) \text { and }\left(\begin{array}{ll}
1 & w \\
0 & 1
\end{array}\right)
$$

and that $\beta$ corresponds to

$$
\left(\begin{array}{ll}
1 & w \\
0 & 1
\end{array}\right)
$$

If $\operatorname{Im}(w)>0$ and $|w|^{2} / 2|\operatorname{Re}(w)|>2$, then $L^{2}$ and $A^{2}$ are given by

$$
L^{2}=\frac{|w|^{2}}{2 \operatorname{Im}(w)} \quad \text { and } \quad A^{2}=\frac{|w|^{2}}{2 \operatorname{Re}(w)} .
$$


For any curve $\gamma \subset M$, let $B \in \operatorname{PSL}(2, \mathbb{C})$ denote the corresponding isometry in $\pi_{1}(M)$. The complex length of $\gamma$ is the value of $\mathcal{L}=l+i \theta$ such that $\operatorname{tr}^{2}(B)=4 \cosh ^{2}(\mathcal{L} / 2)$, $l \geq 0$ and $\theta \in(-\pi, \pi]$. For a geodesic $\gamma$, the real part $l$ gives the length of $\gamma$ in $M$ which is the distance that $B$ translates along its axis. The imaginary part $\theta$ is the amount $B$ rotates about its axis.

Let $\epsilon_{3}$ denote the Margulis constant for $\mathbb{H}^{3}$. If $\gamma$ is the core curve of the solid filling torus in $M$. Then for any $\epsilon_{3} \geq \epsilon>0$, let $\mathbb{T}_{\epsilon}(T)$ (resp. $\mathbb{T}_{\epsilon}(\gamma)$ ) denote the $\epsilon$-Margulis tube about $T$ (resp. $\gamma$ ).

Theorem 1.2 Let $J>1$ and $\epsilon_{3} \geq \epsilon>0$. There is some $K \geq 4 \pi \sqrt{2}$ such that the following holds: suppose $\widehat{M}$ is a geometrically finite hyperbolic 3-manifold with no rank-1 cusps, $T$ is a rank-2 cusp in $\widehat{M}$ and $\beta$ is a slope on $T$ such that the normalized length of $\beta$ is at least $K$ (ie, $L^{2} \geq K^{2}$ ), then

(i) the $\beta$-filling of $\widehat{M}$, which we call $M$, exists;

(ii) the real part of the complex length $\mathcal{L}=l+i \theta$ of the core curve of the filling torus $\gamma$ in $M$ is approximately $2 \pi / L^{2}$ with error bounded by

$$
\left|l-\frac{2 \pi}{L^{2}}\right| \leq \frac{8(2 \pi)^{3}}{L^{4}-16(2 \pi)^{4}} ;
$$

(iii) in particular, the length of $\gamma$ is bounded above by

$$
\frac{2 \pi}{L^{2}-4(2 \pi)^{2}}
$$

(iv) there exists a $J$-bilipschitz diffeomorphism

$$
\phi: \widehat{M}-\mathbb{T}_{\epsilon}(T) \rightarrow M-\mathbb{T}_{\epsilon}(\gamma) ;
$$

(v) if, in addition to $L^{2} \geq K^{2}$, we have $\left|A^{2}\right| \geq 3$, then the imaginary part of the complex length $\mathcal{L}=l+i \theta$ of the core curve of the filling torus $\gamma$ in $M$ (chosen so $\theta \in(-\pi, \pi])$ is approximately $2 \pi / A^{2}$ with error bounded by

$$
\left|\theta-\frac{2 \pi}{A^{2}}\right| \leq \frac{5(2 \pi)^{3}}{\left(L^{2}-4(2 \pi)^{2}\right)^{2}} .
$$

The proof of Theorem 1.2 is contained in Section 4. Although our version may be stated differently, parts (i)-(iii) can be found in the work of Hodgson and Kerckhoff [33; 34] on cone-manifold deformations which was generalized to geometrically finite manifolds by Bromberg [18; 17]. Part (iv) follows from the drilling theorem of Brock and Bromberg [11]. The most original part of this version of the filling theorem is the 
estimate in part (v), although its proof also relies on the Hodgson-Kerckhoff conemanifold technology. Some of the background cone-manifold deformation theory and a summary of the work of Bromberg, Hodgson and Kerckhoff on cone-manifolds can be found in Section 3.

We now outline the proof of Theorem 1.1. We begin by parameterizing a subset of $A H(S \times I)$. If $P \subset S \times\{1\}$ is a pants decomposition, then $M P(S \times I, P)$ denotes the subset of the boundary of $A H(S \times I)$ consisting of the marked hyperbolic 3-manifolds that are homeomorphic to the interior of $S \times I$, are geometrically finite, have a rank-1 cusp associated to each component of $P$ and contain no other cusps (see Section 2 for this notation).

We define a subset $\mathcal{A} \subset M P(S \times I, P) \times \widehat{\mathbb{C}}^{3 g-3}$ and a map

$$
\Phi: \mathcal{A} \rightarrow A H(S \times I),
$$

and we show there is some $\sigma_{0} \in M P(S \times I, P)$ and a neighborhood $U$ of $\left(\sigma_{0}, \infty, \ldots, \infty\right)$ in $\mathcal{A}$ such that $\Phi\left(\sigma_{0}, \infty, \ldots, \infty\right)=\sigma_{0}$ and $\left.\Phi\right|_{U}: U \rightarrow \Phi(U)$ is a homeomorphism. The map $\Phi$ can roughly be described as follows. Let $d=3 g-3$. If $(\sigma, \infty, \ldots, \infty) \in \mathcal{A}$ then we define $\Phi(\sigma, \infty, \ldots, \infty)=\sigma$. If $\left(\sigma, w_{1}, \ldots, w_{d}\right) \in \mathcal{A}$ for some $\left(w_{1}, \ldots, w_{d}\right) \in \mathbb{C}^{d}$, we use the $w$-coordinates to define a marked hyperbolic 3-manifold with $d$ rank-2 cusps. To each rank-2 cusp, one can associate a conformal structure on a torus, and $w_{i}$ acts as a Teichmüller parameter for the $i$-th cusp. We then use the filling theorem (Theorem 1.2) to fill in these cusps and obtain a marked hyperbolic 3-manifold in the interior of $A H(S \times I)$. We define $\mathcal{A}$ to exclude points in $M P(S \times I, P) \times \widehat{\mathbb{C}}^{3 g-3}$ where some, but not all, of the $w$-coordinates are $\infty$.

This parameterization of the subset $\Phi(U) \subset A H(S \times I)$ is a straightforward generalization of the results of Bromberg [20, Section 3]. We set up the necessary background in Section 2 and describe the parameterization in Section 5. This parameterization is an application of parts (i)-(iv) of Theorem 1.2 and Corollary 4.13, which is a generalization of the filling theorem for multiple cusps.

In Section 5.4, we use results of Bromberg [20, Section 4] to show that $\mathcal{A}$ is not locally connected at $\left(\sigma_{0}, \infty, \ldots, \infty\right)$. Moreover, we find that in some neighborhood $U$ of $\left(\sigma_{0}, \infty, \ldots, \infty\right)$ in $\mathcal{A}$, there exists $c>0$ and subsets $C_{n} \subset U$ accumulating at $\left(\sigma_{0}, \infty, \ldots, \infty\right)$ such that for any $\left(\sigma, w_{1}, \ldots, w_{d}\right) \in \overline{C_{n}}$ and any $\left(\sigma^{\prime}, w_{1}^{\prime}, \ldots, w_{d}^{\prime}\right) \in$ $\overline{U-C_{n}}$, we have $\left|w_{1}-w_{1}^{\prime}\right|>c$ for all $n$ (see Lemma 5.8). Heuristically, we think of the sets $C_{n}$ as being components of $U$ that are bounded apart from the rest of $U$ by a lower bound that is independent of $n$. In actuality, these sets are likely collections of components. 
Finally, in Section 6, we show that $A H(S \times I)$ is not locally connected at $\sigma_{0}$. By Lemma 5.8, there is a lower bound on the distance between the first $w$-coordinate (ie, the first coordinate of the $\widehat{\mathbb{C}}^{3 g-d}$ factor of $\mathcal{A}$ ) of a point in $\overline{C_{n}}$ and the first $w-$ coordinate of a point in $\overline{U-C_{n}}$. We then use the filling theorem to estimate the complex length of a curve in $\Phi\left(\sigma, w_{1}, \ldots, w_{d}\right) \in A H(S \times I)$ based on $\left(w_{1}, w_{2}, \ldots, w_{d}\right)$. The control on the $w_{1}$-coordinate from Lemma 5.8 and the quality of the estimates in the filling theorem show that for all but finitely many $n, \overline{\Phi\left(C_{n}\right)}$ and $\overline{\Phi\left(U-C_{n}\right)}$ must be disjoint. Hence, $\overline{\Phi(U)}$ has infinitely many components that accumulate at $\sigma_{0}$. It follows from the Density Theorem (Theorem 2.2) that $\overline{\Phi(U)}$ contains a neighborhood of $\sigma_{0}$ in $A H(S \times I)$; hence, $A H(S \times I)$ is not locally connected at $\sigma_{0}$.

The approach in Section 6 is fundamentally different from Bromberg's [20]. Bromberg uses Minsky's results [61] to extend $\Phi$ continuously to the closure of $\mathcal{A}$. Results of Kerckhoff and Thurston [38] and Brock [10] on the end-invariants in Bers' slices lead us to believe that there is no way of continuously extending $\Phi$ in general. Instead, we use the filling theorem estimates to show that distinct components of $\Phi(U)$ have disjoint closures in $\overline{\Phi(U)}$.

Acknowledgements These results originally formed the content of my $\mathrm{PhD}$ Thesis at the University of Michigan [47]. I owe my advisor, Dick Canary, many thanks for introducing me to this problem, teaching me about deformation spaces and helping me to correct errors and to clarify my work. Initial progress on this work began in 2007 at MSRI, and I thank Juan Souto, Steve Kerckhoff and Ken Bromberg for their direction and many helpful conversations. The results in this paper generalize several different result of Ken Bromberg, and he has been very helpful in explaining his work and intuition. I thank Dave Futer for discussing applications of the filling theorem and catching an important typo. I'd also like to thank the referee for many helpful comments.

The author was partially supported by NSF grants DMS-0902764, DMS-0554239 and DMS-0602191.

\section{Background deformation space theory}

In this section, we recall the definition of a pared 3-manifold $(N, P)$ and define the relative deformation space $A H(N, P)$. This is a space of hyperbolic 3-manifolds homotopy equivalent to $N$ with cusps associated to annuli and tori in $P$. We will review the Ahlfors-Bers parameterization that describes the interior of $A H(N, P)$ and set up some of the notation that will be used later. For more information about pared manifolds and deformation spaces, see Canary and McCullough [27, Chapters 5 and 7], respectively. For a survey of the Density Theorem and bumponomics, see Canary [25]. 


\subsection{Kleinian groups and pared manifolds}

Before turning to deformation spaces, we begin with a brief review of Kleinian group theory in order to set up some notation. A Kleinian group is a discrete subgroup of $\operatorname{PSL}(2, \mathbb{C}) \cong \operatorname{Isom}^{+}\left(\mathbb{H}^{3}\right)$. We will assume that all of our Kleinian groups are finitely generated, torsion-free, and not virtually abelian. The action of a Kleinian group $\Gamma$ on $\mathbb{H}^{3}$ extends to an action on $\partial \mathbb{H}^{3} \cong \widehat{\mathbb{C}}$ by Möbius transformations. The domain of discontinuity $\Omega(\Gamma)$ is the largest open $\Gamma$-invariant subset of $\widehat{\mathbb{C}}$ on which the action of $\Gamma$ is properly discontinuous. The quotient $\Omega(\Gamma) / \Gamma$ is called the conformal boundary of $M_{\Gamma}=\mathbb{H}^{3} / \Gamma$. The limit set, $\Lambda(\Gamma)$, is the complement of $\Omega(\Gamma)$ in $\widehat{\mathbb{C}}$, and the convex core of $M_{\Gamma}$ is the quotient of the convex hull (in $\mathbb{H}^{3}$ ) of the limit set by the group action.

Pared manifolds A pared 3-manifold is a pair $(N, P)$ where $N$ is a compact, oriented, hyperbolizable 3-manifold that is not a 3-ball, and $P \subset \partial N$ is a disjoint collection of incompressible annuli and tori satisfying the following properties:

(1) $P$ contains all of the tori in $\partial N$.

(2) Every $\pi_{1}$-injective map $\left(S^{1} \times I, S^{1} \times \partial I\right) \rightarrow(N, P)$ is homotopic, as a map of pairs, into $P$.

To avoid some degenerate cases in the statements that follow, we will assume throughout this paper that $\pi_{1}(N)$ is not virtually abelian. This will ensure that any Kleinian group isomorphic to $\pi_{1}(N)$ is nonelementary.

Geometrically finite Kleinian groups A hyperbolic 3-manifold $M_{\Gamma}=\mathbb{H}^{3} / \Gamma$ is geometrically finite if and only if the union of $M_{\Gamma}$ with its conformal boundary, $\left(\mathbb{H}^{3} \cup \Omega(\Gamma)\right) / \Gamma$, is homeomorphic to $N-P$ for some pared 3-manifold $(N, P)$. A Kleinian group $\Gamma$ is geometrically finite if and only if the corresponding 3-manifold $M_{\Gamma}$ is geometrically finite. There are many other equivalent notions of geometric finiteness. See Bowditch [9] for a more complete discussion.

Thick-thin decomposition Let $M$ be a hyperbolic manifold. For any $\epsilon>0$, we define the $\epsilon$-thin part of $M$ to be the set of points $x \in M$ where the injectivity radius is at most $\epsilon$ and denote this set by $M \leq \epsilon$. By the Margulis lemma, there is some constant $\epsilon_{3}$ (depending only on the dimension) such that for any $\epsilon_{3} \geq \epsilon>0$, the $\epsilon$-thin part of $M$ consists of a disjoint union of metric collar neighborhoods of short geodesics and cusps. We use the notation $\mathbb{T}_{\epsilon}(\gamma)$ to denote the component of $M^{\leq \epsilon}$ associated to a geodesic $\gamma$ and $\mathbb{T}_{\epsilon}(T)$ to denote the Margulis $\epsilon-$ thin region associated to a rank-2 cusp $T$. We let $\mathbb{T}_{\epsilon}^{\text {par }}$ denote the union of the Margulis $\epsilon$-thin regions associated to parabolics (ie, the rank-1 and rank-2 cusps). 
Klein-Maskit combination Let $H$ be a subgroup of $\Gamma$. A subset $B \subset \widehat{\mathbb{C}}$ is precisely invariant under $H$ in $\Gamma$ if

(1) for all $h \in H, h(B)=B$, and

(2) for all $\gamma \in \Gamma-H, \gamma(B) \cap B=\varnothing$.

For example, if $H$ is the infinite cyclic group generated by

$$
\left(\begin{array}{ll}
1 & 2 \\
0 & 1
\end{array}\right)
$$

and $\Gamma$ is a geometrically finite group containing $H$ with a rank-1 cusp corresponding to $H$ (ie, the largest abelian subgroup of $G$ containing $H$ is $H$ ), then there is some $R$ such that the two sets

$$
B_{R}^{+}=\{z \in \mathbb{C}: \operatorname{Im}(z)>R\} \quad \text { and } \quad B_{R}^{-}=\{z \in \mathbb{C}: \operatorname{Im}(z)<-R\}
$$

are precisely invariant under $H$ in $\Gamma$ (eg, see Marden [50, page 125]).

Precisely invariant sets are useful for constructing Kleinian groups via a process known as Klein-Maskit combination. We will use statements similar to those in Abikoff and Maskit [1], but one should also refer to Maskit [51; 52; 53].

Suppose $G_{1}, G_{2}$ are two geometrically finite Kleinian groups with $G_{1} \cap G_{2}=H$. Here, $H$ could be any subgroup, but we will only be interested in the case that $H$ is the infinite cyclic parabolic subgroup of the previous example. If there is a Jordan curve $c$ bounding two open discs $B_{1}, B_{2}$ in $\widehat{\mathbb{C}}$ such that $B_{i}$ is precisely invariant under $H$ in $G_{i}$, then the group $G$ generated by $G_{1}$ and $G_{2}$ is geometrically finite and isomorphic to the amalgamated free product $G_{1} *_{H} G_{2}$. In this case, we say that the group $G$ is obtained from $G_{1}$ and $G_{2}$ by type I Klein-Maskit combination along the subgroup $H$.

We now describe type II Klein-Maskit combination. Let $G$ be a geometrically finite Kleinian group containing $H$. Let $f \in \operatorname{PSL}(2, \mathbb{C})$ such that $f f^{-1} \subset G$. Suppose there is a Jordan curve $c$ bounding a disc $B \subset \widehat{\mathbb{C}}$ such that

(1) $B$ is precisely invariant for $H$ in $G$,

(2) $\widehat{\mathbb{C}}-f(\bar{B})$ is precisely invariant for $f H f^{-1} \subset G$, and

(3) $g B \cap(\widehat{\mathbb{C}}-f(\bar{B}))=\varnothing$ for all $g \in G$.

Then the group $\Gamma$ generated by $G$ and $f$ is geometrically finite and isomorphic to the HNN extension $G *\langle f\rangle$. 
Again, while type II Klein-Maskit combination can be applied in a more general setting, consider a geometrically finite group $G$ containing $H$ as above, and consider

$$
f=\left(\begin{array}{ll}
1 & w \\
0 & 1
\end{array}\right) \text {. }
$$

Note that $f H f^{-1}=H$. There is some $R$ such that $B_{R}^{-}$and $B_{R}^{+}$are precisely invariant under $H$ in $G$. Moreover, we can assume that for all $g \in G, g B_{R}^{-} \cap B_{R}^{+}=\varnothing$. Then if $\operatorname{Im}(w) \geq 2 R$, the group $G *\langle f\rangle$ is geometrically finite.

\subsection{Deformation spaces}

We define the relative representation variety

$$
\mathcal{R}(N, P)=\operatorname{Hom}_{P}\left(\pi_{1}(N), \operatorname{PSL}(2, \mathbb{C})\right)
$$

to be the set of representations $\rho: \pi_{1}(N) \rightarrow \operatorname{PSL}(2, \mathbb{C})$ such that $\rho(g)$ is parabolic or the identity whenever $g \in \pi_{1}(P)$. We then define the relative character variety $X(N, P)$ to be the Mumford quotient of the relative representation variety

$$
X(N, P)=\mathcal{R}(N, P) / / \operatorname{PSL}(2, \mathbb{C}) .
$$

Although the Mumford quotient is defined algebraically, nonradical points in the character variety can be identified with conjugacy classes of representations (ie, points in the topological quotient $\left.\operatorname{Hom}_{P}\left(\pi_{1}(N), \operatorname{PSL}(2, \mathbb{C})\right) / \operatorname{PSL}(2, \mathbb{C})\right)$ (see Kapovich [36, page 62]). Since we have assumed $\pi_{1}(N)$ is not virtually abelian, $\rho\left(\pi_{1}(N)\right)$ is nonelementary (and therefore nonradical) for any discrete, faithful representation $\rho$. For these representations, we will make no distinction between conjugacy classes of representations and points in $X(N, P)$. See also Culler and Shalen [29, Section 1].

Let $A H(N, P)$ denote the subset of $X(N, P)$ consisting of the conjugacy classes of representations that are discrete and faithful. Thus $A H(N, P)$ inherits a topology from the character variety known as the algebraic topology. Results of Chuckrow [28] and Jørgensen [35] show that $A H(N, P)$ is a closed subset of $X(N, P)$ with respect to this topology. Since $\pi_{1}(N)$ is not virtually abelian, a neighborhood of $A H(N, P)$ is a smooth complex manifold, and the topology on $A H(N, P)$ is the same as the topology when considered as a subset of the topological quotient of $\operatorname{Hom}_{P}\left(\pi_{1}(N), \operatorname{PSL}(2, \mathbb{C})\right)$ by $\operatorname{PSL}(2, \mathbb{C})$ acting by conjugation $[36$, Chapter 4$]$.

The space $A H(N, P)$ is a deformation space of hyperbolic 3-manifolds in the following sense. Given $\rho \in A H(N, P)$, the image group $\rho\left(\pi_{1}(M)\right)$ defines a hyperbolic manifold $M_{\rho}=\mathbb{H}^{3} / \rho\left(\pi_{1}(N)\right)$. Moreover (since $N$ is aspherical) the representation determines a homotopy equivalence $f_{\rho}: N \rightarrow M_{\rho}$, defined up to homotopy. So points in $A H(N, P)$ 
can be identified with equivalence classes of marked hyperbolic 3-manifolds $(M, f)$ where $f: N \rightarrow M$ is a homotopy equivalence such that $f(P)$ is homotopic into the cusps of $M$. Two pairs $\left(M_{1}, f_{1}\right)$ and $\left(M_{2}, f_{2}\right)$ correspond to the same point of $A H(N, P)$ if there is an orientation preserving isometry $g: M_{1} \rightarrow M_{2}$ such that $f_{2} \simeq g \circ f_{1}$.

We say that $\rho \in A H(N, P)$ is minimally parabolic if $\rho(g)$ is parabolic if and only if $g \in \pi_{1}(P)$. A representation $\rho \in A H(N, P)$ is geometrically finite if $\rho\left(\pi_{1}(N)\right)$ is a geometrically finite subgroup of $\operatorname{PSL}(2, \mathbb{C})$. Results of Marden [49] and Sullivan [69] show that when $\partial N-P \neq \varnothing$, the interior of $A H(N, P)$ consists of precisely the conjugacy classes of representations that are both geometrically finite and minimally parabolic, and we denote this set by $\operatorname{MP}(N, P)$. We now describe what is known as the Ahlfors-Bers parameterization of each of the components of $M P(N, P)$ in the case that $\partial N-P$ is incompressible. See [27, Chapter 7] for a more complete description of this parameterization including when $\partial(N, P)$ is compressible.

To enumerate the components of $M P(N, P)$, we first define $A(N, P)$ to be the set of marked pared homeomorphism types. More precisely, $A(N, P)$ is the following set of equivalence classes:

$A(N, P)=\left\{\left[\left(N^{\prime}, P^{\prime}\right), h\right]:\left(N^{\prime}, P^{\prime}\right)\right.$ is a compact, oriented, pared 3-manifold, $h:(N, P) \rightarrow\left(N^{\prime}, P^{\prime}\right)$ is a pared homotopy equivalence $\} / \sim$,

where $\left[\left(N_{1}, P_{1}\right), h_{1}\right] \sim\left[\left(N_{2}, P_{2}\right), h_{2}\right]$ if there exists an orientation preserving pared homeomorphism $j:\left(N_{1}, P_{1}\right) \rightarrow\left(N_{2}, P_{2}\right)$ such that $j \circ h_{1}$ is pared homotopic to $h_{2}$. Recall that we can identify $\rho \in A H(N, P)$ with a marked hyperbolic 3-manifold $\left(M_{\rho}, f_{\rho}\right)$. Any 3-manifold with finitely generated fundamental group admits a compact core by Scott [67]. A relative compact core $C$ of $M_{\rho}$ is a compact core for $M_{\rho}-\mathbb{T}_{\epsilon}^{\text {par }}$ such that $\partial C$ meets every noncompact component of the boundary of $M_{\rho}-\mathbb{T}_{\epsilon}^{\text {par }}$ in an incompressible annulus and contains every toroidal boundary component of $M_{\rho}-\mathbb{T}_{\epsilon}^{\mathrm{par}}$. The existence of a such a core is given by Kulkarni and Shalen [44] and McCullough [55]. This definition naturally imparts a pared structure on any relative compact core whose paring locus consists of the tori and annuli that intersect $\partial \mathbb{T}_{\epsilon}^{\text {par }}$. When $\rho$ is geometrically finite, we can construct a relative compact core $C$ by intersecting the convex core of $M_{\rho}$ with $M_{\rho}-\mathbb{T}_{\epsilon}^{\mathrm{par}}$. We will refer to this as the relative compact core of $M_{\rho}$. If $\rho \in M P(N, P)$ then the marking $f_{\rho}$ is homotopic to a pared homotopy equivalence from $(N, P)$ to the relative compact core of $M_{\rho}$. So we can define a map $F: M P(N, P) \rightarrow$ $A(N, P)$ by sending $\left(M_{\rho}, f_{\rho}\right)$ to the relative compact core of $M_{\rho}$ (still marked by $f_{\rho}$ ). The map $F$ establishes a bijection between the components of $M P(N, P)$ and the elements of the set $A(N, P)$. That is, $F\left(\rho_{1}\right)=F\left(\rho_{2}\right)$ if and only if $\rho_{1}$ and $\rho_{2}$ are in the same component of $M P(N, P)$. 
Let $B$ be the component of $M P(N, P)$ determined by $F^{-1}\left(\left[\left(N^{\prime}, P^{\prime}\right), h\right]\right)$. For $\rho \in B$, we have that $M_{\rho}$ is geometrically finite and minimally parabolic, and $f_{\rho} \circ h^{-1}$ is homotopic to a pared homeomorphism from $\left(N^{\prime}, P^{\prime}\right)$ to the relative compact core of $M_{\rho}$. Using $f_{\rho} \circ h^{-1}$, we can mark each component of the conformal boundary of $M_{\rho}$ with a component of $\partial N^{\prime}-P^{\prime}$. This gives us a map

$$
\mathcal{A B}: B \rightarrow \mathcal{T}\left(\partial N^{\prime}-P^{\prime}\right),
$$

where $\mathcal{T}\left(\partial N^{\prime}-P^{\prime}\right)$ denotes the Teichmüller space of $\partial N^{\prime}-P^{\prime}$. Recall that the Teichmüller space of a disconnected surface is the product of the Teichmüller spaces of its components.

Theorem 2.1 (Ahlfors [3], Bers [8], Kra [42], Marden [49], Maskit [54], Sullivan [69], Thurston [71]) When $\partial N-P$ is incompressible, the map $\mathcal{A B}$ is a homeomorphism on each component of $M P(N, P)$.

Throughout the rest of this paper, we will be primarily concerned with the case $N=$ $S \times I$ where $S$ is a closed surface of genus at least two. In this case, the previous theorem is known as Bers' simultaneous uniformization [7]. The interior of $A H(N)$ (in this case $P=\varnothing$ ) is $M P(N)$ and is connected. The Ahlfors-Bers map defines a homeomorphism

$$
\mathcal{A B}: M P(N) \rightarrow \mathcal{T}(S) \times \mathcal{T}(S) .
$$

Although we will continue to use the term minimally parabolic when $N=S \times I$, representations in $M P(N)$ contain no parabolics.

Generally the Ahlfors-Bers parameterization does not extend over $A H(N, P)$; however, the recent resolution the Bers-Sullivan-Thurston Density Conjecture guarantees that every representation in $A H(N, P)$ can be expressed as the algebraic limit of geometrically finite and minimally parabolic representations. We refer to this as the Density Theorem. In the case that $(N, P)=(S \times I, \varnothing)$, Brock, Canary, and Minsky obtained this result as Corollary 10.1 of the Ending Lamination Theorem [15], using results of Ohshika [64] and Thurston [72].

Theorem 2.2 (Brock-Canary-Minsky [15]) The closure of $M P(S \times I, \varnothing)$, as a subset of the character variety $X(N, P)$, is $A H(S \times I, \varnothing)$.

The more general result that $A H(N, P)=\overline{M P(N, P)}$ for any pared manifold $(N, P)$ follows from work of Brock and Bromberg [11], Brock, Canary and Minsky [14], Bromberg [19], Bromberg and Souto [22], Kim, Lecuire and Ohshika [39], Kleinedam and Souto [40], Lecuire [46], Namazi and Souto [63], Ohshika [65] and Thurston [70]. See Canary [25] for a more complete discussion of the Density Theorem. 
In spite of Theorem 2.1's description of the interior and the Density theorem, the topology of $A H(N, P)$ is more subtle. Anderson and Canary discovered examples of manifolds $N$ for which the components of $M P(N)$ have intersecting closures (often called "bumping") [4]. Since the components of $M P(N)$ are in one-to-one correspondence with the elements of $A(N)$ (ie, marked homeomorphism types) this shows the homeomorphism type of $M_{\rho}$ varies discontinuously in $A H(N)$. Anderson, Canary and McCullough characterized which components of $M P(N)$ bump for any manifold $N$ with incompressible boundary [5].

For $N=S \times I$, the interior $M P(N)$ is connected; hence $A H(N)$ is connected. In this case, McMullen showed a local bumping result: there exists a representation $\rho$ such that whenever $U$ is a sufficiently small neighborhood of $\rho, U \cap M P(N)$ is disconnected by McMullen [59]. Bromberg and Holt generalized this self-bumping result to manifolds containing primitive, essential annuli which are not homotopic into a torus boundary component. In these cases, it follows that $A H(N)$ is not a manifold. The points at which we show $A H(S \times I)$ is not locally connected are self-bumping points; however, in the explicit local parameterization of punctured torus groups given by Bromberg [20], one can find self-bumping points at which the deformation space is locally connected. So the failure of local connectivity also shows $A H(S \times I)$ is not a manifold, but is a finer description of how the end-invariants fail to vary continuously.

All of the known bumping, self-bumping, and local disconnectedness arise from similar constructions involving generalizations of Thurston's hyperbolic Dehn surgery theorem to geometrically finite manifolds [70] (see also [25, Section 7]). One begins with a representation $\rho$ on the boundary with some accidental parabolic $\gamma$ and constructs a new manifold $\hat{N}$ by drilling out $\gamma$. We can pick a hyperbolic structure $\widehat{M}$ on the interior of $\hat{N}$ covered by $\mathbb{H}^{3} / \rho\left(\pi_{1}(N)\right)$. Using the Dehn surgery theorem, we construct a sequence of representations $\rho_{n}$ converging to $\rho$ algebraically such that the corresponding quotient manifolds $M_{n}=\mathbb{H}^{3} / \rho_{n}\left(\pi_{1}(N)\right)$ converge to $\widehat{M}$ geometrically. We construct $\rho_{n}$ as the map induced on $\pi_{1}(N)$ by the composition of immersing $N$ into $\widehat{M}$ followed by Dehn filling the cusp of $\widehat{M}$ where we drilled out $\gamma$. Depending on the topology of $N$, we can usually immerse $N$ in $\widehat{M}$ in different ways by wrapping $N$ around $\gamma$, and this leads to the construction of other sequences accumulating at $\rho$. Various other arguments are then used to analyze how these sequences approach $\rho$ and the local topology of $A H(N)$ near $\rho$.

We do not have a complete classification of the points at which the deformation space bumps or fails to be locally connected. Recently, Brock, Bromberg, Canary and Minsky show there can be no bumping at quasiconformally rigid points (ie, points whose conformal boundary is empty or contains only thrice-punctured spheres). For $N=S \times I$, the deformation space cannot self-bump and therefore is locally connected at 
these points [12]. Evans and Holt are working to find other obstructions to self-bumping based on the length of an accidental parabolic in a conformal boundary component [31].

\section{Background cone-manifold deformation theory}

Let $N$ be a compact 3-manifold. A hyperbolic cone-metric on the interior of $N$ with singular locus consisting of a link $\Sigma \subset \operatorname{int}(N)$ is an incomplete hyperbolic metric (constant sectional curvature equal to -1 ) on the interior of $N-\Sigma$ whose metric completion determines a singular metric on $\operatorname{int}(N)$ with singularities along $\Sigma$. The link is totally geodesic, and in cylindrical coordinates around a component of $\Sigma$, the metric has the form

$$
d r^{2}+\sinh ^{2}(r) d \theta^{2}+\cosh ^{2}(r) d z^{2}
$$

where $\theta$ is measured modulo $\alpha>0$. We require $\alpha$ to be constant on each connected component of $\Sigma$, and we say $\alpha$ is the cone angle about that component of the singular locus. See Hodgson and Kerckhoff [33, Section 1] or Bromberg [18, Section 4] for more details. When the cone angle on each component of $\Sigma$ is $\alpha=2 \pi$, this is equivalent to having a complete hyperbolic metric on the interior of $N$ (ie, in the above definition, we require the metric on $\operatorname{int}(N-\Sigma)$ to be complete in every end of $\operatorname{int}(N-\Sigma)$ not associated to a component of $\Sigma$ ). From now on, we will only consider cone-manifolds whose singular locus is connected.

Let $M_{\alpha}$ be a hyperbolic cone-manifold homeomorphic to the interior of $N$ with cone angle $\alpha$ about $\Sigma$. We now define what it means for $M_{\alpha}$ to be a geometrically finite hyperbolic cone-manifold (see also Section 3 of [18]). To do so, we first define a geometrically finite end. Let $S$ be a closed surface of genus at least two, and let $Y=S \times[0, \infty)$ be a hyperbolic manifold with boundary $S \times\{0\}$. That is, there is a smooth immersion $D: \widetilde{Y} \rightarrow \mathbb{H}^{3}$ and representation $\rho: \pi_{1}(S) \rightarrow \operatorname{PSL}(2, \mathbb{C})$ such that for all $x \in \tilde{Y}$ and $\gamma \in \pi_{1}(S), D(\gamma x)=\rho(\gamma) D(x)$. We say $D$ is the developing map for $Y$ and $\rho$ is the holonomy map. We say $Y$ is a geometrically finite end if $D$ can be extended to a local homeomorphism $\widetilde{S} \times[0, \infty] \rightarrow \mathbb{H}^{3} \cup \widehat{\mathbb{C}}$ such that $D(\widetilde{S} \times\{\infty\}) \subset \widehat{\mathbb{C}}$. In this case, $S \times\{\infty\}$ inherits a conformal structure from the charts defined into $\widehat{\mathbb{C}}$.

Given a hyperbolic cone-manifold $M_{\alpha}$ with cone singularity $\Sigma$, we note that $M_{\alpha}-\Sigma$ has a (possibly incomplete) hyperbolic metric with no cone singularities. Although one could consider hyperbolic cone-manifolds in greater generality, we have defined our cone-manifolds $M_{\alpha}$ to be homeomorphic to the interior of $N$ and hence topologically tame. The ends of $M_{\alpha}-\Sigma$ (ie, the complement of a compact core) are of three types (see [18, page 160]). There will be one end homeomorphic to $T^{2} \times[0, \infty)$ associated to $\Sigma$, some number of ends associated to the rank-2 cusps of $M_{\alpha}$, also homeomorphic 
to $T^{2} \times[0, \infty)$, and some number of ends homeomorphic to $S_{i} \times[0, \infty)$ associated to the higher genus surfaces $S_{i}$ in the boundary of the compact core. We say $M_{\alpha}$ is geometrically finite if each of the ends not associated to a rank-2 cusp or to $\Sigma$ is geometrically finite. We will not be considering hyperbolic cone-manifolds with rank-1 cusps.

We want to provide a meaningful way of interpreting a hyperbolic manifold with a rank-2 cusp as a hyperbolic cone-manifold with cone angle $\alpha=0$ about the conesingularity. The convergence results below will allow us to do this more formally. See also Hodgson and Kerckhoff [34, Section 3] and Bromberg [17, Section 6].

Definition 3.1 A sequence of metric spaces with basepoints $\left\{\left(X_{i}, x_{i}\right)\right\}$ converges to $\left(X_{\infty}, x_{\infty}\right)$ geometrically if, for each $R>0, K>1$, there exists an open neighborhood $U_{\infty}$ of the radius $R$ neighborhood of $x_{\infty}$ in $X_{\infty}$ and some $i_{0}$ such that for all $i>i_{0}$, there is a map $f_{i}:\left(U_{\infty}, x_{\infty}\right) \rightarrow\left(X_{i}, x_{i}\right)$ that is a $K$-bilipschitz diffeomorphism onto its image.

We say that a sequence $X_{i} \rightarrow X_{\infty}$ geometrically if there exist basepoints such that $\left(X_{i}, x_{i}\right) \rightarrow\left(X_{\infty}, x_{\infty}\right)$ geometrically. For a more detailed discussion of geometric convergence in Kleinian group theory, see Benedetti and Petronio [6, Chapter E], Canary, Epstein and Green [26, Chapter I] or Kapovich [36, Chapter 8].

The following is [17, Theorem 6.11], although a finite volume analogue was proven in [34, Section 3].

Theorem 3.2 (Bromberg [17]) Let $\left\{M_{\alpha}\right\}$ be a family of geometrically finite hyperbolic cone-manifold structures on the interior of $N$ defined for $\alpha \in\left(0, \alpha_{0}\right)$, with fixed conformal boundary, $\alpha_{0} \leq 2 \pi$, and suppose there is an embedded tubular neighborhood about the cone-singularity of radius $\geq \sinh ^{-1}(\sqrt{2})$ in $M_{\alpha}$ for all $\alpha \in\left(0, \alpha_{0}\right)$.

(1) As $\alpha \rightarrow 0$, the manifolds $M_{\alpha}$ converge geometrically to a complete hyperbolic manifold $M_{0}$ homeomorphic to the interior of $N-\Sigma$ with a rank-2 cusp in the end associated to $\Sigma$ and the same conformal boundary as $M_{\alpha}$.

(2) As $\alpha \rightarrow \alpha_{0}$, the manifolds $M_{\alpha}$ converge geometrically to a hyperbolic conemanifold $M_{\alpha_{0}}$ with cone angle $\alpha_{0}$ along $\Sigma$ and the same conformal boundary components as $M_{\alpha}$.

This theorem serves two purposes. First, we can interpret a manifold with a rank-2 cusp as a limit of a family of cone-manifolds. Second, a one-parameter family of cone-manifolds $M_{\alpha}$ with fixed conformal boundary, defined for some interval $\left[0, \alpha_{0}\right)$, can be extended to a one-parameter family defined over $\left[0, \alpha_{0}\right]$. 


\subsection{Infinitesimal deformations of cone-manifolds}

Let $X$ denote the interior of $N-\Sigma$ and suppose $M_{t}$ is a one-parameter family of hyperbolic cone-manifolds homeomorphic to the interior of $N$ with singular locus $\Sigma$. By restricting the metric on $M_{t}$ to $X$, we obtain a one-parameter family of hyperbolic metrics on $X$. Up to precomposition by isotopies of $X$ and postcomposition by isometries of $\mathbb{H}^{3}$ this determines a one-parameter family of developing maps

$$
D_{t}: \tilde{X} \rightarrow \mathbb{H}^{3} \text {. }
$$

We will assume $M_{t}$ is smooth in the sense that $D_{t}$ is a smooth one-parameter family of diffeomorphisms.

At each fixed $t$, we obtain a vector field $v$ on $\tilde{X}$ defined by setting $v(x)$ to be the pullback (via $D_{t}$ ) of the tangent vector to the path $t \mapsto D_{t}(x)$. Let $\widetilde{E}=\widetilde{X} \times \mathfrak{s l}_{2}(\mathbb{C})$ be the flat bundle of Killing fields on $\tilde{X}$. In general, unless the deformation is trivial, $v$ will not be a Killing field on $\tilde{X}$; however, we can associate to $v$ the Killing field, or equivalently the section $s_{v}: \tilde{X} \rightarrow \widetilde{E}$ of the bundle $\widetilde{E}$, that best approximates $v$ at $x$. To define $s_{v}$ we first need to analyze the natural complex structure $\widetilde{E}$ inherits from $\mathfrak{s l}_{2}(\mathbb{C})$.

At each point $x \in \tilde{X}$, the fiber of $\widetilde{E}$ decomposes as a direct sum $\widetilde{\mathcal{P}} \oplus \widetilde{\mathcal{K}}$ where $\widetilde{\mathcal{P}}$ consists of the infinitesimal isometries which are pure translations and $\widetilde{\mathcal{K}}$ consists of the infinitesimal isometries that are pure rotations at $x$. See [33, pages 13-19]. The sub-bundle $\tilde{\mathcal{P}}$ is naturally identified with $T \tilde{X}$, and using the complex structure of $\widetilde{E}$ one sees that $\tilde{\mathcal{K}}=i \tilde{\mathcal{P}}$, so we can decompose $\widetilde{E} \cong T \tilde{X}+i T \tilde{X}$. With this notation, we can define the canonical lift of the vector field $v$ to be the section $s_{v}: \tilde{X} \rightarrow \widetilde{E}$ given by

$$
s_{v}(x)=v(x)-i \operatorname{curl}(v)(x)
$$

Here we are using twice the usual curl, which is normally defined by $\operatorname{curl}(v)=$ $\frac{1}{2}(* \widehat{d} \hat{v})$ where $\hat{d}$ is exterior differentiation and $\hat{v}$ is the 1 -form dual to $v$. Under the identification of Killing fields with $\mathfrak{s l}_{2}(\mathbb{C})$, this curl operator on vector fields acts like multiplication by $i$ on sections of $\widetilde{E}$. Hence, $s_{v}$ is the section whose real part agrees with $v$ and such that the real part of $\operatorname{curl}(s)$ agrees with $\operatorname{curl}(v)$. See $[33 ; 18]$ for more details.

Recall, an $\widetilde{E}$-valued $k$-form (on $\tilde{X}$ ) is a section of the bundle $\bigwedge^{k} T \tilde{X}^{*} \otimes \widetilde{E} \rightarrow \tilde{X}$, and the exterior derivative $d: \bigwedge^{k} T \tilde{X}^{*} \otimes \widetilde{E} \rightarrow \bigwedge^{k+1} T \tilde{X}^{*} \otimes \widetilde{E}$ is defined using the flat connection on $\widetilde{E}$. Let $E$ be the flat bundle obtained by taking the quotient of $\widetilde{E}$ by $\pi_{1}(X)$ where $\pi_{1}(X)$ acts on $\tilde{X}$ by covering transformations and on $\mathfrak{s l}_{2}(\mathbb{C})$ by the adjoint representation. We define $E$-valued $k$-forms similarly to $\widetilde{E}$-valued forms, 
and using the exterior derivative, we can define the cohomology groups $H^{k}(X ; E)$ to be the set of closed forms, denoted $\Omega^{k}(X ; E)$, modulo the exact forms.

If $s_{v}$ is the canonical lift of a vector field $v$ as defined above, then $d s_{v}$ is an equivariant closed 1 -form and thus descends to an element $\omega \in \Omega^{1}(X ; E)$. Moreover, the cohomology class in $H^{1}(X ; E)$ defined by $\omega$ is independent of the choice of developing maps $D_{t}$. This is proven in [33, Section 2] (see also [18, Section 2]). As Hodgson and Kerckhoff explain in [34, page 375], altering $D_{t}$ by postcomposing by an isometry of $\mathbb{H}^{3}$ has no effect on $\omega$ and precomposing by an isotopy of $\tilde{X}$ only affects $\omega$ by an exact form. Thus the cohomology class determined by $\omega$ is well-defined by the one-parameter family of metrics on $X$. Conversely, every cohomology class determines an infinitesimal change in the metric on $X$ [33, page 12-13].

\subsection{Standard forms and Hodge forms}

For each cohomology class, we now define two particular closed forms representing this class: a standard form which will take a particular structure in the cusps of $X$ and a Hodge representative.

Hodgson and Kerckhoff calculated the effects of two particular $E$-valued 1-forms $\omega_{m}$ and $\omega_{l}$ in a neighborhood of $\Sigma$. The former represents an infinitesimal deformation which changes the cone angle about $\Sigma$, and the latter changes the length of the singular locus. See [33, pages 12-13] for precise definitions of these forms.

Definition 3.3 A closed $E$-valued 1-form $\omega$ is in standard form if there is a neighborhood $U_{1}$ of the singular locus and neighborhoods $U_{2}, \ldots, U_{n}$ of each rank-2 cusp such that in $U_{i}, \omega$ equals a complex linear combination of $\omega_{m}$ and $\omega_{l}$.

Note that the complex coefficients of $\omega_{m}$ and $\omega_{l}$ will generally be different for each $U_{i}$. The following lemma [33, Lemma 3.3] shows that every closed form in $\Omega^{1}(X ; E)$ can be put into standard form.

Lemma 3.4 (Hodgson-Kerckhoff [33]) Given any closed E-valued 1-form $\phi$, there is a cohomologous form $\omega_{0}$ that is in standard form.

Note that standard forms are not unique since there are no restrictions outside the union of the neighborhoods $U_{i}$. Next we find a Hodge representative in every cohomology class and show its difference from a standard form is bounded.

There is a natural inner product on the fibers of $E$ coming from the hyperbolic metric on $X$ (see [18, Section 2] for formulas). Using this inner product, we have an 
isomorphism $\sharp: E \rightarrow E^{*}$. We define an inner product on $E$-valued $k$-forms by

$$
\left(\omega_{1}, \omega_{2}\right)=\int_{X} \omega_{1} \wedge\left(\sharp * \omega_{2}\right),
$$

where $*$ denotes the Hodge star operator. Let $\delta$ denote the adjoint of $d$ with respect to this inner product. We will use the conventional notation $\|\omega\|^{2}=(\omega, \omega)$ and say $\omega$ is in $L^{2}$ if $\|\omega\|$ is finite. See [16, pages $13-14 ; 33$, Section $\left.1 ; 18 ; 17\right]$ for formulas for $d, \delta$ and a broader development of this theory.

Definition 3.5 A 1-form $\omega \in \Omega^{1}(X ; E)$ is a Hodge form if $\omega$ is closed (ie, $\left.d \omega=0\right)$, co-closed (ie, $\delta \omega=0$ ), and locally $\omega$ can be expressed as $d s$ where $s$ is the canonical lift of a divergence-free, harmonic vector field.

Before stating [18, Theorem 4.3] which generalizes [33, Theorem 2.7], we need to define what it means for a 1-form to be conformal at infinity. By [18, Lemma 3.2], there is an isomorphism $\Pi_{*}: H^{1}(X ; E) \rightarrow H^{1}\left(\partial_{c} X ; E_{\infty}\right)$ where $\partial_{c} X$ is the conformal boundary of $X$ and $E_{\infty}$ is the bundle of germs of projective vector fields on $\partial_{c} X$. A cohomology class $\omega_{\infty} \in H^{1}\left(\partial_{c} X ; E_{\infty}\right)$ is conformal if it can be expressed as $d s_{\infty}$ where $s_{\infty}$ is the canonical lift of an automorphic, conformal vector field on $\partial_{c} X$. A cohomology class $\omega \in H^{1}(X ; E)$ is conformal at infinity if $\Pi_{*}(\omega)$ is conformal.

We will only be concerned with 1 -forms on $X$ that arise from one-parameter deformations of hyperbolic cone manifolds $M_{t}$ whose conformal boundary is fixed throughout the deformation. These 1 -forms will be conformal at infinity with respect to the definition given above.

Theorem 3.6 (Bromberg [18], Hodgson-Kerckhoff [33]) Let $M$ be a geometrically finite hyperbolic cone-manifold, and let $\omega_{0}$ be an $E$-valued 1-form on $X=M-\Sigma$ that is conformal at infinity and in standard form in a neighborhood $U$ of $\Sigma$. Then there exists a unique Hodge form $\omega$ such that the following holds:

(1) $\omega$ is cohomologous to $\omega_{0}$.

(2) There exists an $L^{2}$ section $s$ of $E$ such that $d s=\omega_{0}-\omega$.

(3) $\omega_{0}-\omega$ has finite $L^{2}$ norm on the complement of $U$.

Using their analysis of Hodge forms and infinitesimal deformations, Bromberg generalized the local rigidity results of Hodgson and Kerckhoff [33] to show that the (possibly incomplete) hyperbolic metric on the interior of a cone-manifold $M_{\alpha}$ is completely determined by the cone angle $\alpha$ and the conformal boundary components associated to each of the geometrically finite ends of $M_{\alpha}$. See [18, Theorem 5.8] which is restated as [17, Theorem 1.1]. We will use the following consequence of Bromberg's result. 
Theorem 3.7 (Bromberg [18]) Let $M_{\alpha_{0}}$ be a geometrically finite hyperbolic conemanifold with cone angle $\alpha_{0} \in[0,2 \pi]$ about the cone singularity $\Sigma$. Suppose there is an embedded tubular neighborhood about $\Sigma$ in $M_{\alpha_{0}}$ of radius $\geq \sinh ^{-1}(\sqrt{2})$. Then there exists an open neighborhood $W$ of $\alpha_{0}$ in $[0,2 \pi]$ such that the one-parameter family $M_{\alpha}$, defined by varying the cone angle and keeping the conformal boundary of $M_{\alpha_{0}}$ fixed, is defined for all $\alpha \in W$.

\subsection{Complex length}

Let $X$ be the interior of $N-\Sigma$ and $\rho: \pi_{1}(X) \rightarrow \operatorname{PSL}(2, \mathbb{C})$. If $\gamma \in \pi_{1}(X)$, then the complex length of $\gamma$, denoted $\mathcal{L}=l+i \theta$ or $\mathcal{L}(\rho(\gamma))$, is defined by the formula

$$
\operatorname{tr}^{2}(\rho(\gamma))=4 \cosh ^{2}\left(\frac{\mathcal{L}}{2}\right)
$$

and the normalizations $l \geq 0$ and $\theta \in(-\pi, \pi]$. If $\rho(\gamma)$ is a loxodromic element, then $l$ is the length of the geodesic representative of $\gamma$ in $X$ (equivalently, the translation length of $\rho(\gamma)$ along its axis in $\mathbb{H}^{3}$ ), and $\theta$ gives the amount $\rho(\gamma)$ twists along its axis. If $\rho(\gamma)$ is parabolic, then the complex length is zero.

If $M_{\alpha}$ is a cone-manifold homeomorphic to the interior of $N$ with cone singularity $\Sigma$, and $U$ is a tubular neighborhood of the cone-singularity, then $\partial U$ has a well-defined meridian $\beta$. This is the homotopy class of a curve on $\partial U$ that bounds a disk (with a cone-point) in $M_{\alpha}$. When the cone angle is $\alpha \in(0,2 \pi)$, then the meridian will be sent to an elliptic element that rotates by $\alpha$ about its axis. In this case, we say the meridian has (purely imaginary) complex length $i \alpha$. In our situation, when $\alpha=0$ we will have $\rho(\beta)$ be parabolic, but when $\alpha=2 \pi, \rho(\beta)$ will be the identity.

If $\alpha \in(0,2 \pi]$ and $U$ is a metric collar neighborhood, then the torus $\partial U$ inherits a Euclidean metric as a subset of $M_{\alpha}$, so we can pick the shortest longitude $\lambda$ on $\partial U$ (by a longitude, we mean any curve that intersects the meridian once) and normalize the complex length of $\lambda$ to be $l+i \theta$ for some $\theta \in(-\alpha / 2, \alpha / 2]$. Then any other longitude will have complex length $l+i \theta+i m \alpha$ for some $m \in \mathbb{Z}$. The only time the choice of $\lambda$ is not well-defined is when there are two shortest longitudes on $\partial U$ in which case we pick one and assign it the complex length $l+i(\alpha / 2)$ by convention. We say that the complex length of the cone axis $\Sigma$ is the complex length of any longitude since these are all homotopic to $\Sigma$. Since the complex length of $\Sigma$ is only well-defined up to the addition of multiples of $i \alpha$, we work with the complex length of a particular longitude instead. 


\section{The drilling and filling theorems}

\subsection{Drilling}

If $M$ is a geometrically finite hyperbolic manifold and $\gamma$ is a disjoint collection of simple closed geodesics in $M$, then Kojima showed, using an argument he attributes to Kerckhoff, that $M-\gamma$ admits a unique complete hyperbolic metric such that the natural inclusion of $(M-\gamma) \subset M$ extends to a conformal map between the conformal boundaries of $M-\gamma$ and $M$ (see Kojima [41, Theorem 1.2.1]). We call this process drilling (ie, finding a new metric on $M-\gamma$ ). If the curves in $\gamma$ are sufficiently short, the Brock-Bromberg drilling theorem bounds the difference between the original metric on the complement of $\gamma$ in $M$ and the new complete metric on $M-\gamma$ [11]. That is, they show the metrics are close (in a bilipschitz sense) on the complement of a neighborhood of the drilled curves. Their work makes use of the cone-manifold machinery developed by Hodgson and Kerckhoff [33; 34] and Bromberg [18; 17] that we outlined in the previous section.

More precisely, if $M$ is a geometrically finite hyperbolic manifold without rank-1 cusps, and $\gamma_{1}, \ldots, \gamma_{n}$ is a disjoint collection of simple closed curves in $M$, let $\widehat{M}$ denote the geometrically finite hyperbolic manifold homeomorphic that is homeomorphic $M-\bigcup \gamma_{i}$ and has the same conformal boundary components. We say $\widehat{M}$ is the $\bigcup \gamma_{i}$-drilling of $M$. The following is [11, Theorem 6.2].

Theorem 4.1 (Brock-Bromberg [11]) Given any $J>1, \epsilon_{3} \geq \epsilon>0$, there exists some $l_{0}>0$ such that the following holds: if $M$ is a geometrically finite manifold with no rank-1 cusps, and $\gamma_{1}, \ldots, \gamma_{n}$ is a collection of geodesics in $M$ with

$$
\sum_{i=1}^{n} l\left(\gamma_{i}\right)<l_{0},
$$

then there exists a $J$-bilipschitz diffeomorphism

$$
\phi: \widehat{M}-\bigcup_{i=1}^{n} \mathbb{T}_{\epsilon}\left(T_{i}\right) \rightarrow M-\bigcup_{i=1}^{n} \mathbb{T}_{\epsilon}\left(\gamma_{i}\right),
$$

where $\widehat{M}$ is the $\bigcup \gamma_{i}$-drilling of $M$, and $T_{i}$ is the cusp corresponding to the drilling of $\gamma_{i}$.

Remark In the drilling theorem, Brock and Bromberg also conclude that the map $\phi$ in the drilling theorem is level-preserving on cusps in the following sense. Suppose $T_{\widehat{M}}$ is a rank-2 cusp in $\widehat{M}-\bigcup_{i=1}^{n} \mathbb{T}_{\epsilon}\left(T_{i}\right)$. Then the drilling map $\phi$ sends $T_{\widehat{M}}$ to a cusp $T_{M}$ in $M$ in such a way that for any $0<\epsilon^{\prime} \leq \epsilon, \phi\left(\partial \mathbb{T}_{\epsilon^{\prime}}\left(T_{\widehat{M}}\right)\right)=\partial \mathbb{T}_{\epsilon^{\prime}}\left(T_{M}\right)$. See [11, Theorem 6.12] (in particular, see [11, Lemma 6.17] which was used to prove Theorem 6.12). 


\subsection{Filling}

The filling theorem provides an inverse construction. A geometrically finite hyperbolic manifold with a rank-2 cusp is homeomorphic to the interior of a compact manifold with a torus boundary component. One can Dehn-fill this compactification along any boundary slope and attempt to hyperbolize the interior of the filled manifold. Under certain conditions on the boundary slope, the filling theorem provides a way of doing this hyperbolic Dehn-filling while preserving the conformal boundary components. Moreover, we obtain estimates on the complex length of the core curve of the filling torus in the new metric.

Now let $\widehat{M}$ be a geometrically finite hyperbolic manifold with $n$ rank-2 cusps. We want to describe a way of filling in these cusps to obtain a hyperbolic manifold $M$ with the same conformal boundary but no rank-2 cusps. Although methods developed by Hodgson, Kerckhoff and Purcell [66] can be used to fill multiple cusps simultaneously, this introduces some unnecessary complications. We will proceed by describing the filling theorem for one cusp, which up to renumbering we can assume is the first cusp. Then we derive the multiple cusp case by filling one cusp at a time (see Corollary 4.13).

Let $\widehat{N}$ be a compact 3-manifold with interior homeomorphic to $\widehat{M}$. On the first torus boundary component of $\hat{N}$ fix a slope $\beta$. Let $N$ be the manifold obtained by Dehn-filling $\hat{N}$ along $\beta$. If possible, we hyperbolize the interior of $N$ to obtain a hyperbolic manifold $M$ with the same conformal boundary as $\widehat{M}$ and one fewer cusp. If it exists, we call $M$ the $\beta$-filling of $\widehat{M}$. Let $\gamma$ be the geodesic representative in $M$ of the core curve of the solid torus used to Dehn-fill $\hat{N}$.

The following theorem gives sufficient conditions for the $\beta$-filling of $\widehat{M}$ to exist, and when these conditions are satisfied, gives information about the complex length of the geodesic $\gamma$ in $M$. Before stating the theorem, we define the normalized length and normalized twist of the slope $\beta$ used in the filling; henceforth called the meridian.

The boundary of the cusp $T$ we are filling, $\partial \mathbb{T}_{\epsilon_{3}}(T)$, inherits a Euclidean metric. Let $\mu$ be a geodesic in the homotopy class of $\beta$ on this flat torus. Let $m$ denote the length of $\mu$. Fix a point $x \in \mu$, and let $v$ be a geodesic ray perpendicular to $\mu$ at $x$. Let $y$ denote the next point on $v$ where $v$ meets $\mu$ (after $x$ ). Orient $\mu$ so that if $\vec{\mu}$ and $\vec{v}$ denote the tangent vectors to $\mu$ and $v$ at $x$ then $\vec{\mu} \times \vec{v}$ points into the cusp $T$. Define $b$ to be the value in $(-m / 2, m / 2]$ such that $|b|$ is the distance between $x$ and $y$, and after orienting $\mu$ as described, the sign of $b$ gives the orientation of the shortest path beginning at $x$ and ending at $y$ realizing this distance. See Figure 1 . When $y$ is exactly half-way around $\mu$ from $x$ then there are two shortest paths. In this case, we choose the positively oriented one so $b=m / 2$. The value $b$ is the twist associated to the flat structure on $\partial \mathbb{T}_{\epsilon_{3}}(T)$ with meridian $\beta$. 


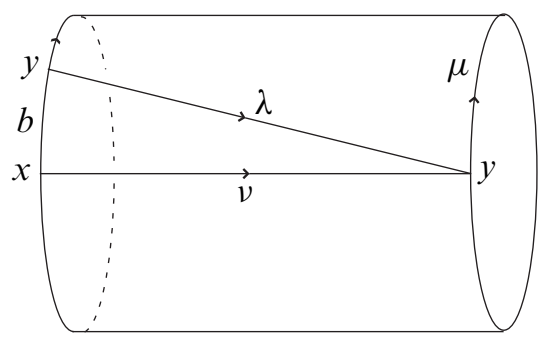

Figure 1: A flat torus obtained by identifying the boundary circles of a cylinder with a twist of $b$. In this picture, $b$ is positive.

Definition 4.2 The normalized length $L$ of $\beta$ is the length of $\mu$ divided by the square root of the area of $\partial \mathbb{T}_{\epsilon_{3}}(T)$ :

$$
L=\frac{m}{\sqrt{\operatorname{Area}\left(\partial \mathbb{T}_{\epsilon_{3}}(T)\right)}} .
$$

Definition 4.3 The normalized twist of the flat metric on $\partial \mathbb{T}_{\epsilon_{3}}(T)$ with meridian $\beta$ is the ratio $b / m$. We let $A^{2}=m / b$ denote the reciprocal of the normalized twist.

Remark The normalized length and normalized twist are well-defined invariants of the cusp $T$ with meridian $\beta$. That is, they depend only on the point in $\mathcal{T}\left(T^{2}\right) / \mathbb{Z}$ determined by the flat metric on $\partial \mathbb{T}_{\epsilon_{3}}(T)$. Here we consider the quotient of the Teichmüller space of the torus by the subgroup generated by Dehn twists about $\beta$. In particular, we could define the normalized length or twist using any cross section of the cusp $\partial \mathbb{T}_{\epsilon}(T)$, where $\epsilon_{3} \geq \epsilon>0$.

Also note that in spite of the "square" notation, the quantity $A^{2} \in(-2,2]$ could be negative, and we make no use of any quantity $A$ in this paper. We use this notation to emphasize that $A^{2}$ is a counterpart to $L^{2}$ in the following sense.

Assume $A^{2} \neq 2$ so there is a unique shortest longitude $\lambda$ on $T^{2} \times\{0\}$. If we conjugate $\pi_{1}(\widehat{M})$ in $\operatorname{PSL}(2, \mathbb{C})$ so that the isometry corresponding to $\lambda$ is

$$
\left(\begin{array}{ll}
1 & 2 \\
0 & 1
\end{array}\right)
$$

and the isometry corresponding to the meridian $\beta$ is

$$
\left(\begin{array}{ll}
1 & w \\
0 & 1
\end{array}\right)
$$


for some $w \in \mathbb{C}$ with $\operatorname{Im}(w)>0$, then

$$
L^{2}=\frac{|w|^{2}}{2 \operatorname{Im}(w)} \quad \text { and } \quad A^{2}=\frac{|w|^{2}}{2 \operatorname{Re}(w)} .
$$

Now that we have defined the quantities $L^{2}$ and $A^{2}$, we are ready to state the filling theorem.

Theorem 1.2 Let $J>1$ and $\epsilon_{3} \geq \epsilon>0$. There is some $K \geq 4 \pi \sqrt{2}$ such that the following holds: suppose $\widehat{M}$ is a geometrically finite hyperbolic 3-manifold with no rank-1 cusps, $T$ is a rank-2 cusp in $\widehat{M}$, and $\beta$ is a slope on $T$ such that the normalized length of $\beta$ is at least $K$ (ie, $L^{2} \geq K^{2}$ ), then

(i) the $\beta$-filling of $\widehat{M}$, which we call $M$, exists,

(ii) the real part of the complex length $\mathcal{L}=l+i \theta$ of the core curve of the filling torus $\gamma$ in $M$ is approximately $2 \pi / L^{2}$ with error bounded by

$$
\left|l-\frac{2 \pi}{L^{2}}\right| \leq \frac{8(2 \pi)^{3}}{L^{4}-16(2 \pi)^{4}},
$$

(iii) in particular, the length of $\gamma$ is bounded above by

$$
\frac{2 \pi}{L^{2}-4(2 \pi)^{2}}
$$

(iv) there exists a $J$-bilipschitz diffeomorphism

$$
\phi: \widehat{M}-\mathbb{T}_{\epsilon}(T) \rightarrow M-\mathbb{T}_{\epsilon}(\gamma),
$$

(v) if, in addition to $L^{2} \geq K^{2}$, we have $\left|A^{2}\right| \geq 3$, then the imaginary part of the complex length $\mathcal{L}=l+i \theta$ of the core curve of the filling torus $\gamma$ in $M$ (chosen so $\theta \in(-\pi, \pi])$ is approximately $2 \pi / A^{2}$ with error bounded by

$$
\left|\theta-\frac{2 \pi}{A^{2}}\right| \leq \frac{5(2 \pi)^{3}}{\left(L^{2}-4(2 \pi)^{2}\right)^{2}} .
$$

Outline of the proof and prior results As the proof is rather technical and spans multiple subsections, we begin with an outline. Many of the results of the filling theorem follow directly from results of Brock, Bromberg, Hodgson, and Kerckhoff, so first we delineate these contributions. Part (i) was shown by Bromberg in [17]. This was a generalization of the work of Hodgson and Kerckhoff in [34] to geometrically finite manifolds. We intend to revisit these arguments in order to reprove (i) and draw the conclusions stated in (ii) and (v). Part (iii) follows from (ii), although both are proven in Lemma 4.7. Part (iv) follows from parts (i), (iii) and the drilling theorem. 
That is, once we show that the filling exists and $\gamma$ is sufficiently short in $M$, we can then apply the drilling theorem to $M$ to recover $\widehat{M}$ and get the $J$-bilipschitz map from the drilling theorem.

We view the geometrically finite manifold $\widehat{M}$ with $n$ cusps as a geometrically finite hyperbolic cone-manifold with $n-1$ rank-2 cusps and cone singularity $\Sigma=\gamma$ with cone angle $\alpha=0$ about $\Sigma$. Part (i) amounts to showing that we can increase the cone angle from 0 to $2 \pi$. We will use the parameterization $t=\alpha^{2}$. By Theorem 3.7 the oneparameter family is defined in some interval $\left[0, t^{\prime}\right)$. At any $t \in\left[0, t^{\prime}\right)$, Proposition 4.4 provides estimates on the derivative of the complex length of any peripheral curve. The bounds here depend on the radius of an embedded tube about the cone-singularity. Provided $L^{2}$ is sufficiently large, Lemma 4.5 shows that for all $t \in\left[0, t^{\prime}\right)$, the radius remains bounded below; therefore, we can extend the one-parameter family to $M_{t^{\prime}}$ using Theorem 3.2, and in Lemma 4.6 we show that if $L^{2} \geq 8(2 \pi)^{2}$ then we can continue the deformation to $t=(2 \pi)^{2}$. Having defined the one-parameter family $M_{t}$ for all $t \in\left[0,(2 \pi)^{2}\right]$, we can integrate the estimates from Proposition 4.4 to prove Lemma 4.7. This gives us the estimate on the real part of the length of $\gamma$ we claimed in part (ii).

To get the estimate on the imaginary part of the complex length of $\gamma$ in $M$ that we claimed in part (v), we consider a longitude on $\partial U_{1}$ homotopic to $\gamma$. Actually, it is more convenient to work with a metric collar neighborhood $V_{t}$ of $\gamma$ in $M_{t}$. The torus $\partial V_{t}$ inherits a flat metric and we can define the twist $b(t)$ and the normalized twist $b(t) / m(t)$ associated to $\partial V_{t}$ in the same way that we defined the twist $b$ and normalized twist $b / m$ for $\partial V_{0}$ (the boundary of the cusp we are filling in $\widehat{M}$ ). Since $\left|A^{2}\right|>2$, there is a unique shortest longitude $\lambda$ on $\partial V_{0}$. For any $t$ when $\lambda$ is shortest, the imaginary part of the complex length which we denote by $\theta(\lambda, t)$ is well-defined as a real number (see Section 3.3). In Lemma 4.8, we define the quantity $v\left(\lambda^{\prime}, t\right)=\theta\left(\lambda^{\prime}, t\right) / \alpha$ for any longitude $\lambda^{\prime}$ and bound $d v / d \alpha$ at any fixed time $t$. In Lemma 4.10, we show $\lim _{t \rightarrow 0} v(\lambda, t)=1 / A^{2}$. In Lemma 4.11 and Lemma 4.12 we use this limiting value of $v(\lambda, t)$, the bound on the derivative of $v$ found in Lemma 4.8, and the hypothesis $\left|A^{2}\right| \geq 3$ to show that $\lambda$ remains the shortest longitude on $\partial V_{t}$ for all $t \in\left[0,(2 \pi)^{2}\right]$ and obtain the estimate on $\theta=\theta(\lambda, 2 \pi)$ in part (v) of Theorem 1.2.

\subsection{Existence of the deformation and derivative estimates}

We now begin the proof of Theorem 1.2. In this section, our goal is to show that the one-parameter family $M_{t}$, defined by setting $M_{0}=\widehat{M}$ and increasing the cone angle about $\gamma$ with the parameterization $t=\alpha^{2}$ (while keeping the conformal boundary fixed), can be defined for all $t \in\left[0,(2 \pi)^{2}\right]$. The deformation is well defined for some 
interval $\left[0, t^{\prime}\right.$ ) by the local rigidity results of Bromberg (see [18, Theorem 5.8], restated as Theorem 3.7 in the previous section). At any $t \in\left[0, t^{\prime}\right)$ we can estimate the derivative of the complex length of any curve on the boundary of a neighborhood of the cone singularity.

Proposition 4.4 Suppose the one-parameter family of cone manifolds $M_{t}$ has been defined for $t \in\left[0, t^{\prime}\right)$, and suppose there is an embedded tube $U_{1}$ of radius $R$ about $\gamma$ in $M_{t}$. If $\mathcal{L}=l+i \theta$ denotes the complex length of any curve on $\partial U_{1}$, then the derivative of $\mathcal{L}$ is given by

$$
\frac{d \mathcal{L}}{d t}=\frac{-1}{4 \alpha^{2}}(-2 \mathcal{L})+(x+i y)(2 l)
$$

and therefore

$$
\frac{d l}{d \alpha}=\frac{l}{\alpha}\left(1+4 \alpha^{2} x\right) \quad \text { and } \quad \frac{d \theta}{d \alpha}=\frac{\theta}{\alpha}+4 \alpha y l,
$$

where $x$ and $y$ satisfy

$$
\begin{aligned}
\frac{-1}{\sinh ^{2}(R)}\left(\frac{2 \sinh ^{2}(R)+1}{2 \sinh ^{2}(R)+3}\right) \leq 4 \alpha^{2} x & \leq \frac{1}{\sinh ^{2}(R)} \\
\left|4 \alpha^{2} y\right| & \leq \frac{2}{\sinh ^{2}(R)} \frac{\cosh ^{2}(R)}{\left(2 \cosh ^{2}(R)+1\right)} .
\end{aligned}
$$

Proof Let $X=M_{t}-\Sigma$. To each time $t$ for which the one-parameter deformation has been defined we can associate a class in $H^{1}(X ; E)$ that is conformal at infinity. We can represent this cohomology class in two ways. First, by Lemma 3.4, we can choose $\omega_{0}$ to be in standard form in a neighborhood $U_{1}$ of the singular locus and in each of the rank-2 cusps $U_{i}(i=2, \ldots, n)$ of $X$. By our choice of parameterization $t=\alpha^{2}$, in a neighborhood $U_{1}$ we must have

$$
\omega_{0}=\frac{-1}{4 \alpha^{2}} \omega_{m}+(x+i y) \omega_{l}
$$

for some constants $x$ and $y$ since the derivative of the complex length of the meridian is determined by the coefficient of $\omega_{m}$ and the complex length of the meridian is $i \sqrt{t}$ at any time $t$ (see [34, Equation (4)]). By integrating $\omega_{m}$ and $\omega_{l}$, Hodgson and Kerckhoff compute the effect of $\omega_{m}$ and $\omega_{l}$ on the infinitesimal change in the holonomy of any path on $\partial U_{1}$ in [33, pages 32-33] (see also [34, Lemma 2.1]). Thus (1) follows from (5) by their work.

From (1), the real part gives us $d l / d t$ and the imaginary part gives us $d \theta / d t$. Given the parameterization $t=\alpha^{2}$, the formulas for $d l / d \alpha$ and $d \theta / d \alpha$ given in (2) follow. 
Now we want to derive the bounds on $x$ and $y$. By Theorem 3.6, we can find a Hodge form $\omega$ in the same cohomology class as $\omega_{0}$ such that $\omega_{c}:=\omega-\omega_{0}$ has finite $L^{2}$-norm outside $U_{1}$. Lemma 3.4 of [33] shows that $\omega_{c}$ does not effect the holonomy of any of the peripheral elements. By Proposition 2.6 of [33], we can write $\omega$ as

$$
\omega=\eta+i * D \eta
$$

such that $D^{*} \eta=D * D \eta+\eta=0$, and both $\eta$ and $D \eta$ are symmetric and traceless $T X$-valued forms. Here, $D$ is the real part of $d$, and $D^{*}$ is the adjoint of $D$; see [33; 18] for formulas.

Since $\omega=\omega_{0}+\omega_{c}$, we can decompose the real part of $\omega$ as $\eta=\eta_{0}+\eta_{c}$ where $\eta_{c}$ does not effect the holonomy of the peripheral elements.

We can find a smoothly embedded convex surface $S$ cutting off the geometrically finite ends of $X$ such that (after possibly shrinking the $U_{i}$ neighborhoods of the cusps) $U_{i}$ and $S$ are all pairwise disjoint. Note that such a surface exists by [18, Theorem 4.3] and may have multiple components (one for each end).

By [34, Lemma 2.3], we have that for any compact submanifold $N \subset X$ with $\partial N$ oriented with an inward pointing normal

$$
\int_{N}\|\omega\|^{2}=\int_{\partial N} * D \eta \wedge \eta
$$

Letting $N$ be the complement of the union $\bigcup_{i=1}^{n} U_{i}$ and the ends cut off by $S$, we can decompose the boundary integral:

$$
\int_{N}\|\omega\|^{2}-\int_{S} * D \eta \wedge \eta=\sum_{i=1}^{n} \int_{\partial U_{i}} * D \eta \wedge \eta
$$

Let $b_{i}(\alpha, \beta)=\int_{\partial U_{i}} * D \alpha \wedge \beta$.

Lemma 2.5 of [34] says that $b_{i}(\eta, \eta)=b_{i}\left(\eta_{0}, \eta_{0}\right)+b_{i}\left(\eta_{c}, \eta_{c}\right)$ and Lemma 2.6 of [34] says that $b_{i}\left(\eta_{c}, \eta_{c}\right)$ is nonpositive. So we have

$$
\int_{N}\|\omega\|^{2}-\int_{S} * D \eta \wedge \eta \leq \sum_{i=1}^{n} b_{i}\left(\eta_{0}, \eta_{0}\right) .
$$

Since this holds for any compact submanifold $N$, we can apply this to a family of compact submanifolds obtained by shrinking the neighborhood of the geometrically finite ends (but keeping the torus boundary components of $N$ the same as before). Let $S_{T}$ be the surface obtained by taking a parallel copy of $S$ a distance $T$ further out the 
ends, and $N_{T}$ the submanifold containing $N$ whose boundary is $S_{T}$. Then for all $T$,

$$
\int_{N_{T}}\|\omega\|^{2}-\int_{S_{T}} * D \eta \wedge \eta \leq \sum_{i=1}^{n} b_{i}\left(\eta_{0}, \eta_{0}\right) .
$$

The calculations in the proof of [18, Theorem 4.6] show that $\int_{S_{T}} * D \eta \wedge \eta \rightarrow 0$ as $T \rightarrow \infty$. Since $N_{T}$ contains the original manifold $N=N_{0}$, for all $T$ we have $\int_{N_{T}}\|\omega\|^{2} \geq \int_{N}\|\omega\|^{2} \geq 0$.

As Purcell calculates in [66, Equation (16)], this shows

$$
\sum_{i=1}^{n} b_{i}\left(\eta_{0}, \eta_{0}\right) \geq 0
$$

with equality if and only if the deformation is trivial.

We also note that Lemmas 3.1 and 3.2 in [66] imply that for $i \geq 2$,

$$
-b_{i}\left(\eta_{0}, \eta_{0}\right)=2\left|\zeta_{i}^{\prime}(t)\right|^{2} \operatorname{Area}\left(\partial U_{i}\right)
$$

where $\zeta_{i}(t)$ is the path in the Teichmüller space (using the Teichmüller metric) of $\partial U_{i}$ throughout the deformation. In particular $b_{i}\left(\eta_{0}, \eta_{0}\right) \leq 0$ for all $i \geq 2$. It follows that $b_{1}\left(\eta_{0}, \eta_{0}\right) \geq 0$. We now are in a position to follow the calculations in [34, pages 382384] identically (for more steps in the calculations see [34; 47, Chapter 4]).

In order to bound $x$ and $y$, we begin by computing $b_{1}\left(\eta_{0}, \eta_{0}\right)$ in terms of $x$ and $y$ and some constants that only depend on $R$ and $\alpha$ (within this proposition, $R$ and $\alpha$ are fixed so we refer to $a_{R}, b_{R}, c_{R}$ as constants). This is done in [34, 382-383].

$$
\frac{b_{1}\left(\eta_{0}, \eta_{0}\right)}{\operatorname{Area}(\partial V)}=a_{R}\left(x^{2}+y^{2}\right)+b_{R} x+c_{R}
$$

where

$$
\begin{aligned}
a_{R} & =-\tanh (R) \frac{2 \cosh ^{2}(R)+1}{\cosh ^{2}(R)}, \\
b_{R} & =\frac{\tanh (R)}{2 \alpha^{2} \cosh ^{2}(R) \sinh ^{2}(R)}, \\
c_{R} & =\frac{\tanh (R)+\tanh ^{3}(R)}{16 \alpha^{4} \sinh ^{4}(R)} .
\end{aligned}
$$

Using the fact that $b_{1}\left(\eta_{0}, \eta_{0}\right) \geq 0$, we get

$$
\left(x+\frac{b_{R}}{2 a_{R}}\right)^{2}+y^{2} \leq \frac{b_{R}^{2}-4 a_{R} c_{R}}{4 a_{R}^{2}} .
$$


Since both of the terms $\left(x+b_{R} /\left(2 a_{R}\right)\right)^{2}$ and $y^{2}$ are positive, we get the inequalities

$$
\begin{aligned}
\left(x-\frac{1}{\left(4 \alpha^{2} \sinh ^{2}(R)\right)\left(2 \cosh ^{2}(R)+1\right)}\right)^{2} & \leq\left(\frac{\cosh ^{2}(R)}{\left(2 \alpha^{2} \sinh ^{2}(R)\right)\left(2 \cosh ^{2}(R)+1\right)}\right)^{2}, \\
y^{2} & \leq\left(\frac{\cosh ^{2}(R)}{\left(2 \alpha^{2} \sinh ^{2}(R)\right)\left(2 \cosh ^{2}(R)+1\right)}\right)^{2} .
\end{aligned}
$$

The inequalities (3) and (4) follow immediately. This completes the proof which gives us a generalized version of [34, Theorem 2.7] for geometrically finite manifolds with rank-2 cusps. Moreover, we obtain an additional bound on $y$ which will be useful later.

Because the hypotheses in Proposition 4.4 require the existence of an embedded tube $U_{1}$ of radius $R$ about $\gamma$, we now show there is some interval on which there is a lower bound to the size of such a tube. From now on we will use $R_{t}$ to denote the maximum radius of an embedded tubular neighborhood about $\gamma$ in $M_{t}$, and let $V_{t}$ denote this $R_{t}$-neighborhood of $\gamma$. Note that this replaces the neighborhood $U_{1}$ we were using earlier because we are now interested in the parameter $t$ and no longer care about the other neighborhoods $U_{i}, i \geq 2$. Although not necessarily the optimal lower bound, it will be convenient to show $R_{t} \geq \sinh ^{-1}(\sqrt{2})$. In particular, this will allow us to invoke [17, Theorem 1.2] in the proof of Lemma 4.6. (See the comments preceding [17, Theorem 3.5, page 796].)

Lemma 4.5 Suppose $M_{t}$ is defined for some interval $\left[0, t^{\prime}\right) \subset\left[0,(2 \pi)^{2}\right]$, and let $R_{t}$ denote the maximal radius such that if $V_{t}$ is an $R_{t}$-neighborhood of $\Sigma$ in $M_{t}$ then $V_{t}$ is embedded. Suppose $L^{2} \geq 8(2 \pi)^{2}$ where $L$ is the normalized length of the meridian of $\partial V_{0}$. Then $R_{t}>\sinh ^{-1}(\sqrt{2})$ for all $t \in\left[0, t^{\prime}\right)$.

Proof When $t=0, M_{0}$ has a rank-2 cusp. We can interpret $V_{0}$ as this rank-2 cusp and $R_{0}=\infty$. As we vary the metric, $R_{t}$ varies continuously. Suppose there was some first time $t^{\prime \prime}<t^{\prime}$ such that $R_{t^{\prime \prime}}=\sinh ^{-1}(\sqrt{2})$. Let $l(t)$ denote the length of $\gamma$ in $M_{t}$. This is the same as the length of any curve on $\partial V_{t}$ homotopic to $\gamma$ so we can apply the bounds on $d l / d t$ in Proposition 4.4. We will find a contradiction by showing that $R_{t^{\prime \prime}}$ is bounded below by a function of $l\left(t^{\prime \prime}\right)$ and estimate $l\left(t^{\prime \prime}\right)$ using $L^{2}$.

The area $A_{t}$ of $\partial V_{t}$ satisfies

$$
A_{t} \geq 1.69785 \frac{\sinh ^{2}\left(R_{t}\right)}{\cosh \left(2 R_{t}\right)}
$$


by [34, Theorem 4.4] (see also [17, Proposition 3.4] in the geometrically finite setting). The value 1.69785 is an approximation of $2 \sqrt{6} \sinh ^{-1}(1 /(2 \sqrt{2}))$ but we won't need this precision. Define

$$
h(r)=1.69785 \frac{\tanh (r)}{\cosh (2 r)} .
$$

Remark Hodgson and Kerckhoff define $h(r)=3.3957(\tanh (r) / \cosh (2 r))$ in [34, Section 5]. Our definition differs by a factor of 2 since we are allowing our manifold to have multiple cusps and geometrically finite ends (see [34, Theorem 4.4]). This also allows us to directly apply [17, Propositions 3.2 and 3.4].

Since $A_{t}=\alpha l(t) \sinh \left(R_{t}\right) \cosh \left(R_{t}\right)$ [34, page 403], the maximal radius $R_{t}$ satisfies

$$
\alpha l(t) \geq h\left(R_{t}\right) .
$$

In [34, Lemma 5.2], Hodgson and Kerckhoff show that $h$ has a unique maximum, $h_{\max } \approx 0.5098$, when $r \approx 0.531$ and is decreasing for all $r \geq 0.531$. For any $0 \leq a \leq h_{\max }$ we can define an inverse function $h^{-1}(a)$ to be the value of $r$ such that $r \geq 0.531$ and $h(r)=a$. One can easily see from the definition of $h(r)$ that $\lim _{r \rightarrow \infty} h(r)=0$, so we interpret $h^{-1}(0)=\infty$.

Then we have

$$
R_{t} \geq h^{-1}(\alpha l(t))
$$

whenever $\alpha l(t) \leq h_{\max }$ and $R_{t} \geq 0.531$. We are assuming $R_{t} \geq \sinh ^{-1}(\sqrt{2}) \approx 1.4622$ for all $0 \leq t \leq t^{\prime \prime}$ so the condition that $R_{t} \geq 0.531$ is immediately satisfied for all $t$ in this interval.

If $\alpha l(t) \leq h_{\max }$, set $\rho(t)=h^{-1}(\alpha l(t))$ which is clearly bounded above by $R_{t}$. Note that $\alpha$ and $l(t)$ both start at zero when $t=0$ so the condition that $\alpha l(t) \leq h_{\max }$ holds in some interval around $t=0$.

Substituting $\rho$ for $R_{t}$ in the inequality (3) gives us

$$
\frac{-1}{\sinh ^{2}(\rho)}\left(\frac{2 \sinh ^{2}(\rho)+1}{2 \sinh ^{2}(\rho)+3}\right) \leq 4 \alpha^{2} x \leq \frac{1}{\sinh ^{2}(\rho)} .
$$

Proposition 5.5 of [34] shows the lower bound is an increasing function of $\rho$ and the upper bound is a decreasing function. Now set

$$
u(t)=\frac{\alpha}{l(t)} .
$$

Differentiating with respect to $t$, we find that

$$
\frac{d u}{d t}=\frac{-1}{2 \alpha l}\left(4 \alpha^{2} x\right)
$$


and after the substitution $z=\tanh (\rho)$,

$$
-\frac{1+z^{2}}{3.3956\left(z^{3}\right)} \leq \frac{d u}{d t} \leq \frac{\left(1+z^{2}\right)^{2}}{3.3956\left(z^{3}\right)\left(3-z^{2}\right)} .
$$

As long as $\alpha l \leq h_{\max }$ we have $0.531 \leq \rho=h^{-1}(\alpha l)$ and therefore $0.48 \leq z \leq 1$. Since

$$
\frac{1+z^{2}}{3.3957\left(z^{3}\right)} \text { and } \frac{\left(1+z^{2}\right)^{2}}{3.3957\left(z^{3}\right)\left(3-z^{2}\right)}
$$

are both decreasing functions of $z$ over this interval, we can replace $z$ with 0.48 to obtain the somewhat liberal bound

$$
\left|\frac{d u}{d t}\right| \leq 4
$$

Equation (37) of [34] shows that $\lim _{\alpha \rightarrow 0} u=L^{2}$ which implies that as long as $\alpha l(t) \leq h_{\max }$ and $0 \leq t \leq t^{\prime \prime}$ we have

$$
\left|\frac{\alpha}{l(t)}-L^{2}\right| \leq 4 t
$$

Since $L^{2} \geq 8(2 \pi)^{2}$, we have that $L^{2} \pm 4 t$ is positive for any $t \leq(2 \pi)^{2}$, so

$$
\frac{\alpha}{L^{2}+4 t} \leq l(t) \leq \frac{\alpha}{L^{2}-4 t}
$$

Multiplying by $\alpha$ and substituting $t=\alpha^{2}$ we get

$$
\alpha l(t) \leq \frac{t}{L^{2}-4 t} .
$$

Since $L^{2} \geq 8(2 \pi)^{2}$ we have $L^{2}-4(2 \pi)^{2} \geq 4(2 \pi)^{2}$. Thus

$$
\frac{(2 \pi)^{2}}{L^{2}-4(2 \pi)^{2}} \leq \frac{1}{4}<h_{\max }
$$

This implies that for any $0 \leq t \leq(2 \pi)^{2}$,

$$
\frac{t}{L^{2}-4 t} \leq \frac{(2 \pi)^{2}}{L^{2}-4(2 \pi)^{2}}<h_{\max }
$$

which in particular implies that $\alpha l(t)<h_{\max }$ for all $t \in\left[0, t^{\prime \prime}\right]$. It also follows that

$$
R_{t^{\prime \prime}} \geq h^{-1}\left(\alpha l\left(t^{\prime \prime}\right)\right) \geq h^{-1}\left(\frac{t^{\prime \prime}}{L^{2}-4 t^{\prime \prime}}\right) \geq h^{-1}\left(\frac{(2 \pi)^{2}}{L^{2}-4(2 \pi)^{2}}\right) \geq h^{-1}\left(\frac{1}{4}\right)
$$


Since $\frac{1}{4}<h\left(\sinh ^{-1}(\sqrt{2})\right) \approx 0.27725, R_{t^{\prime \prime}}>\sinh ^{-1}(\sqrt{2})$. This contradicts that $R_{t^{\prime \prime}}=\sinh ^{-1}(\sqrt{2})$ for any time $t^{\prime \prime}<t^{\prime}$, and so we have $R_{t}>\sinh ^{-1}(\sqrt{2})$ for all $0 \leq t<t^{\prime}$.

Remark Although many of these estimates appear in [34; 17], we will be using them to produce estimates in following subsection which do not appear in the existing literature.

Using Theorem 3.2 and Theorem 3.7, we can extend the one-parameter family $M_{t}$ to be defined for all $t \in\left[0,(2 \pi)^{2}\right]$. The following lemma proves part (i) of the filling theorem. See [17, Theorem 1.2] for the proof.

Lemma 4.6 If $L^{2} \geq 8(2 \pi)^{2}$, then the one-parameter family is defined for all $t \in$ $\left[0,(2 \pi)^{2}\right]$.

\subsection{Complex length estimates}

Now that we have defined the one-parameter family for all $t \in\left[0,(2 \pi)^{2}\right]$, we can integrate the estimates we found for $d l / d \alpha$ and $d \theta / d \alpha$ in Proposition 4.4. This will allow us to estimate the complex length of any longitude on $\partial V_{t}$ at any $t$. When $t=(2 \pi)^{2}$, this produces estimates on the complex length of $\gamma$ in $M$.

First we consider the real part of the complex length of $\gamma$. As in the proof of Lemma 4.5, we consider $u(t)=\alpha / l(t)$, which approaches $L^{2}$ as $t \rightarrow 0$. We can integrate the bounds on $d u / d t$ in (7) to estimate the length of $\gamma$ in $M$. In other words, the inequalities in (8) hold for all $t \in\left[0,(2 \pi)^{2}\right]$. Thus we have shown:

Lemma 4.7 If $L^{2} \geq 8(2 \pi)^{2}$, the length of $\gamma$ in $M$ is given by $l\left((2 \pi)^{2}\right)$ which satisfies

$$
\frac{2 \pi}{L^{2}+4(2 \pi)^{2}} \leq l\left((2 \pi)^{2}\right) \leq \frac{2 \pi}{L^{2}-4(2 \pi)^{2}} .
$$

This immediately implies parts (ii) and (iii) of the filling theorem.

Next we consider the imaginary part of the complex length of $\gamma$. Again we consider any longitude on $\partial V_{t}$. Recall from Section 3.3 that for a fixed $t$ we can choose a shortest longitude on $\partial V_{t}$ and thus identify the imaginary part of the complex length of any longitude on $\partial V_{t}$ with a real number as opposed to modulo $\alpha$. We begin by using the bounds from Proposition 4.4 to bound the change in $\theta(t) / \alpha$ for any longitude on $\partial V_{t}$. 
Lemma 4.8 Suppose $L^{2} \geq 8(2 \pi)^{2}$. For any fixed $t$ such that $0<t \leq(2 \pi)^{2}$ and any longitude on $\partial V_{t}$, let $\mathcal{L}=l+i \theta$ denote the complex length of that longitude. Define

$$
v=\frac{\theta}{\alpha} .
$$

Then

$$
\left|\frac{d v}{d \alpha}\right| \leq \frac{5(2 \pi)}{\left(L^{2}-4(2 \pi)^{2}\right)^{2}} .
$$

Remark Because we are considering the derivative at a fixed time $t$, we use $l=l(t)$ to denote the length at time $t$ and $\theta=\theta(t)$. Also note that the role of $v$ is similar to that of the reciprocal of $u$, rather than $u$ itself.

Proof Because $L^{2} \geq 8(2 \pi)^{2}$, the one-parameter family $M_{t}$ can be defined for all $t \in\left[0,(2 \pi)^{2}\right]$ by Lemma 4.6. Lemma 4.5 implies that $R_{t} \geq \sinh ^{-1}(\sqrt{2})$ for all $t$, and so we can apply the results of Proposition 4.4 with a lower bound on $R$.

Recall from (2) in the statement of Proposition 4.4 that $d \theta / d \alpha=\theta / \alpha+4 \alpha y l$, so differentiating $v$ with respect to $\alpha$ gives us

$$
\frac{d v}{d \alpha}=\frac{(d \theta / d \alpha)}{\alpha}-\frac{\theta}{\alpha^{2}}=4 y l .
$$

In order to obtain a bound on this quantity, we rewrite $4 y l$ in the following way since we can bound $\left|4 \alpha^{2} y\right|$ using (4).

$$
\left|\frac{d v}{d \alpha}\right|=|4 y l|=\left|\frac{l^{2}}{\alpha^{2} l} 4 \alpha^{2} y\right|=(l)\left(\frac{1}{u}\right)\left(\frac{1}{\alpha l}\right)\left|4 \alpha^{2} y\right| .
$$

We will bound each of these four quantities separately. First, an upper bound for $l$ at any time $t$ is given in (8). For any $t \leq(2 \pi)^{2}$ this upper bound satisfies the uniform bound

$$
l \leq \frac{\alpha}{L^{2}-4 t} \leq \frac{2 \pi}{L^{2}-4(2 \pi)^{2}}
$$

Recall that $u=\alpha / l$ and so the quantity $1 / u=l / \alpha$ can bounded using (7). Since $u$ approaches $L^{2}$ as $\alpha \rightarrow 0$ and $|d u / d t| \leq 4$, we have that $\left|u-L^{2}\right| \leq 4 \alpha^{2}$. Therefore at any $t \leq(2 \pi)^{2}$, a lower bound for $u$ is given by $u \geq L^{2}-4(2 \pi)^{2}$ which implies

$$
\frac{1}{u} \leq \frac{1}{L^{2}-4(2 \pi)^{2}}
$$


Making the same changes of variables $\rho=h^{-1}(\alpha l)$ and $z=\tanh (\rho)$ that we made in the proof of the inequality (6) in Proposition 4.4, we see that (as in [34, Equation (38)])

$$
\frac{1}{\alpha l}=\frac{1+z^{2}}{1.69785(z)\left(1-z^{2}\right)} .
$$

Finally we must bound $4 \alpha^{2} y$ in terms of $z$. As in the proof of Proposition 4.4, we can replace $R$ by $\rho$ in the inequality (4) to get

$$
\left|4 \alpha^{2} y\right| \leq \frac{2}{\sinh ^{2}(\rho)} \frac{\cosh ^{2}(\rho)}{\left(2 \cosh ^{2}(\rho)+1\right)} .
$$

Using $\sinh ^{2}(\rho)=z^{2} /\left(1-z^{2}\right)$ and $\cosh ^{2}(\rho)=1 /\left(1-z^{2}\right)$ gives us

$$
\left|4 \alpha^{2} y\right| \leq \frac{2\left(1-z^{2}\right)}{3 z^{2}-z^{4}} .
$$

Now we combine the bounds on $l, 1 / u, 1 /(\alpha l)$ and $\left|4 \alpha^{2} y\right|$ in (11), (12), (13) and (14) to get an estimate replacing (10):

$$
\left|\frac{d v}{d \alpha}\right| \leq\left(\frac{2 \pi}{L^{2}-4(2 \pi)^{2}}\right)\left(\frac{1}{L^{2}-4(2 \pi)^{2}}\right)\left(\frac{1+z^{2}}{1.69785(z)\left(1-z^{2}\right)}\right)\left(\frac{2\left(1-z^{2}\right)}{3 z^{2}-z^{4}}\right) .
$$

Since $L^{2} \geq 8(2 \pi)^{2}$ we know (as in the proof of Lemma 4.5) that $\alpha l$ remains bounded above by $h_{\max }$ for all $t$ and therefore $\rho \geq 0.531$. This implies $1 \geq z \geq 0.4862$ throughout the deformation so we can bound the following function of $z$ by its value when $z=0.48$ since it is decreasing on $[0.48,1]$.

$$
\frac{2\left(1+z^{2}\right)\left(1-z^{2}\right)}{1.69785(z)\left(1-z^{2}\right)\left(3 z^{2}-z^{4}\right)} \leq \frac{2\left(1+(0.48)^{2}\right)\left(1-(0.48)^{2}\right)}{1.69785(0.48)\left(1-(0.48)^{2}\right)\left(3(0.48)^{2}-(0.48)^{4}\right)} .
$$

This upper bound is approximately 4.73191 , so for any $z \in[0.48,1]$,

$$
\frac{2\left(1+z^{2}\right)\left(1-z^{2}\right)}{1.69785(z)\left(1-z^{2}\right)\left(3 z^{2}-z^{4}\right)} \leq 5 \text {. }
$$

Thus

$$
\left|\frac{d v}{d \alpha}\right| \leq \frac{5(2 \pi)}{\left(L^{2}-4(2 \pi)^{2}\right)^{2}} .
$$

The quantity $v=v\left(\lambda^{\prime}, t\right)$ depends on both a longitude $\lambda^{\prime}$ and the parameter $t$. The previous Lemma shows that if $L^{2}$ is sufficiently large, then $v$ remains roughly constant. Thus for any longitude $\lambda^{\prime}, v\left(\lambda^{\prime},(2 \pi)^{2}\right)$ can be approximated by $\lim _{t \rightarrow 0} v\left(\lambda^{\prime}, t\right)$. We claim that since $\left|A^{2}\right|>2$, there is a longitude $\lambda$ such that this limit exists and is equal to $1 / A^{2}$, the normalized twist of the cusp we are filling $\widehat{M}$. 
Let $b(t)$ and $m(t)$ be the twist and length of the meridian on the flat torus $V_{t}$, as in Definition 4.3. Since $M_{t} \rightarrow M_{0}$ geometrically, the flat tori $\partial V_{t}$ converge to $\partial V_{0}$. Hence $b(t) / m(t)$ converges to $b(0) / m(0)=1 / A^{2}$ unless $b / m=\frac{1}{2}$ (in which case $\lim (b(t) / m(t))$ could be $\frac{1}{2},-\frac{1}{2}$, or not exist).

Lemma 4.9 Suppose that $\left|A^{2}\right|>2$. Then

$$
\lim _{t \rightarrow 0} \frac{b(t)}{m(t)}=\frac{1}{A^{2}} .
$$

When $b(t) / m(t) \neq \frac{1}{2}$, then there is a unique shortest longitude $\lambda$ on $\partial V_{t}$. In particular, if $\left|A^{2}\right|>2$, there is a unique shortest longitude $\lambda$ on $\partial V_{0}$.

Lemma 4.10 If $\left|A^{2}\right|>2$, then there is some $\delta>0$ such that the imaginary part of the complex length of the shortest longitude $\lambda$ lies in the interval $\theta(\lambda, t) \in(-\alpha / 2, \alpha / 2)$ for all $0<t<\delta$. Moreover,

$$
\lim _{t \rightarrow 0} v(\lambda, t)=\lim _{t \rightarrow 0} \frac{\theta(\lambda, t)}{\alpha}=\frac{1}{A^{2}} .
$$

Proof Since $\left|1 / A^{2}\right|<\frac{1}{2}$, there is some $\delta>0$ such that for all $t \in[0, \delta), b(t) / m(t) \neq \frac{1}{2}$ by the previous lemma. Hence, the longitude $\lambda$ that is shortest on $\partial V_{0}$ is the unique shortest longitude on $\partial V_{t}$ for all $t \in[0, \delta)$. Thus, the imaginary part of the complex length satisfies $\theta(\lambda, t) \in(-\alpha / 2, \alpha / 2)$ for all $t \in(0, \delta)$.

For any $t \in(0, \delta)$, we have $\alpha>0$, so the ratio $\theta(\lambda, t) / \alpha$ is the amount the shortest longitude $\lambda$ twists around the meridian, measured in the interval $\left(-\frac{1}{2}, \frac{1}{2}\right]$. Given the definitions of $b(t)$ and $m(t)$, this ratio is identical to $b(t) / m(t)$. Hence,

$$
\frac{\theta(\lambda, t)}{\alpha}=\frac{b(t)}{m(t)}
$$

Thus

$$
\lim _{t \rightarrow 0} v(\lambda, t)=\lim _{t \rightarrow 0} \frac{\theta(\lambda, t)}{\alpha}=\lim _{t \rightarrow 0} \frac{b(t)}{m(t)}=\frac{1}{A^{2}} .
$$

We now use the bounds on $d v / d \alpha$ and the fact that $\lim _{t \rightarrow 0} v(\lambda, t)=1 / A^{2}$ to estimate $v\left(\lambda,(2 \pi)^{2}\right)$.

Lemma 4.11 Suppose that $\left|A^{2}\right|>2$ and $L^{2} \geq 8(2 \pi)^{2}$. Then the complex length $l\left(\lambda,(2 \pi)^{2}\right)+i \theta\left(\lambda,(2 \pi)^{2}\right)$ of the longitude $\lambda$ on $\partial V_{(2 \pi)^{2}}$ satisfies

$$
\left|\theta\left(\lambda,(2 \pi)^{2}\right)-\frac{2 \pi}{A^{2}}\right| \leq \frac{5(2 \pi)^{3}}{\left(L^{2}-4(2 \pi)^{2}\right)^{2}} .
$$


Proof Recall that for any longitude, we obtained the bound

$$
\left|\frac{d v}{d \alpha}\right| \leq \frac{5(2 \pi)}{\left(L^{2}-4(2 \pi)^{2}\right)^{2}}
$$

in Lemma 4.8. For the longitude $\lambda$, we have $\lim _{t \rightarrow 0} v(\lambda, t)=1 / A^{2}$, so we can integrate to find that, for any $t \leq(2 \pi)^{2}$, we have

$$
\left|\frac{\theta(\lambda, t)}{\alpha}-\frac{1}{A^{2}}\right| \leq \frac{5(2 \pi)(t)}{\left(L^{2}-4(2 \pi)^{2}\right)^{2}} .
$$

Since $L^{2} \geq 8(2 \pi)^{2}$ we can define the deformation for all $t \in\left[0,(2 \pi)^{2}\right]$, and therefore setting $t=(2 \pi)^{2}$ gives us inequality (15).

We can now prove part (v) of Theorem 1.2.

Lemma 4.12 Let $\gamma$ be the core curve of the filling torus in $M=M_{(2 \pi)^{2}}$. If $L^{2} \geq 8(2 \pi)^{2}$ and $\left|A^{2}\right| \geq 3$, then the imaginary part of the complex length $\mathcal{L}(\gamma)=$ $l(\gamma)+i \theta(\gamma)$, normalized so that $\theta(\gamma) \in(-\pi, \pi]$, satisfies

$$
\left|\theta(\gamma)-\frac{2 \pi}{A^{2}}\right| \leq \frac{5(2 \pi)^{3}}{\left(L^{2}-4(2 \pi)^{2}\right)^{2}} .
$$

Proof Since any longitude on $\partial V_{(2 \pi)^{2}}$ is homotopic to $\gamma$, the complex length $l(\gamma)+i \theta(\gamma)$ of $\gamma$ in $M_{(2 \pi)^{2}}$ is given by the complex length of the shortest longitude $\lambda$ on $\partial V_{(2 \pi)^{2}}$. Since $\left|A^{2}\right| \geq 3$, there is a unique shortest longitude at $t=0$. The conditions $\left|A^{2}\right| \geq 3, L^{2} \geq 8(2 \pi)^{2}$, together with inequality (16) show that $\theta(\lambda, t) / \alpha$ remains in the open interval $\left(-\frac{1}{2}, \frac{1}{2}\right.$ ) for all $t \leq(2 \pi)^{2}$ (ie, at no time does $\theta(\lambda, t) / \alpha$ equal $\pm \frac{1}{2}$ and $\lambda$ ceases to be the unique shortest longitude). Thus, $\lambda$ remains the shortest longitude throughout the deformation and $\theta\left(\lambda,(2 \pi)^{2}\right)$ lies in the interval $(-\pi, \pi)$. So the normalized $\theta(\gamma)$ is $\theta\left(\lambda,(2 \pi)^{2}\right)$ and inequality (17) follows directly from (15).

We now complete the proof of the filling theorem by summarizing what we have done to prove parts (i), (ii), (iii), and deriving part (iv). Part (i) was proven in Lemma 4.6 when we showed that one can increase the cone angle from 0 to $2 \pi$. Parts (ii) and (iii) were completed in Lemma 4.7. Part (iv) follows from parts (i), (iii), and the drilling theorem (Theorem 4.1) in the following way. Part (i) provides the existence of $M$ (ie, the $\beta$-filling of $\widehat{M}$ ), and by the drilling theorem, there exists some $l_{0}$ such that if $l(\gamma)<l_{0}$ then there is a $J$-bilipschitz diffeomorphism

$$
\phi: \widehat{M}-\mathbb{T}_{\epsilon}(T) \rightarrow M-\mathbb{T}_{\epsilon}(\gamma) .
$$


By part (iii) of the filling theorem, there exists some $K$ such that if the normalized length, $L$, of $\beta$ is at least $K$ then $l(\gamma) \leq 2 \pi /\left(L^{2}-4(2 \pi)^{2}\right)<l_{0}$. Thus we can apply the drilling theorem to reverse the filling and obtain the desired bilipschitz map.

Remark Note that in parts (i), (ii), (iii), (v) of the filling theorem, we only used the uniform bounds $L^{2} \geq 8(2 \pi)^{2}$ and $\left|A^{2}\right| \geq 3$. These four parts do not depend on the condition that $L \geq K$. The constant $K$ depends on $J$ and $\epsilon$ and is therefore only necessary to conclude that if $L \geq K$, then the map $\phi$ is $J$-bilipschitz outside a Margulis $\epsilon$-thin region about the cusp $T$. We also remark that since the filling map $\phi$ is obtained by applying the drilling theorem, we can assume that $\phi$ is level-preserving on cusps (see the remark following Theorem 4.1).

Before moving on to the next section, we remark that one could eliminate the hypothesis that $\left|A^{2}\right| \geq 3$ by not requiring the normalization $\theta(\gamma) \in(-\pi, \pi]$. For instance, if we only assume $\left|A^{2}\right|>2$, then (15) still holds. If $A^{2}=2$, then Lemma 4.8 could still be used to estimate the imaginary part of the complex length of $\gamma$ using an altered definition of the normalized twist (see the remarks in [47, Section 4.3]).

\subsection{Consequences and generalizations}

In this section, we explain some of the consequences of the drilling and filling theorems. First, we draw the following corollary of Theorem 4.1 and Theorem 1.2 that will allow us to fill a manifold with multiple cusps. If $\widehat{M}$ has multiple cusps, we can fill them one at a time, using the filling theorem each time to obtain bounds on the lengths of the core curves of the filling tori. In the statement of the following corollary, we will suppose our manifold has $d$ cusps of which $n \leq d$ are being filled. We will assume they have been ordered so that the first $n$ are filled. We label the cusps in $\widehat{M}$ by $T_{i}$ and we label the core curves of the solid tori by $\gamma_{i}$.

Corollary 4.13 Let $J>1, l_{0}>0, \epsilon_{3} \geq \epsilon>0$, and $n$ be given. There exists some $K$ such that the following holds: suppose $\widehat{M}$ is a geometrically finite manifold with $d \geq n$ cusps. Suppose $\beta_{i}$ is a slope on the $i$-th cusp of $\widehat{M}, 1 \leq i \leq n \leq d$. If the normalized length of $\beta_{i}$ is at least $K$ for each $i$, then

(i) we can fill in the $n$ cusps with labeled meridians, obtaining a manifold $M$ such that each $\beta_{i}$ bounds a disk in $M$ (in other words, the $\bigcup \beta_{i}$-filling of $\widehat{M}$ exists),

(ii) if $\gamma_{i}$ is the core curve of the torus used to fill the $i$-th cusp, then $\sum_{i=1}^{n} l\left(\gamma_{i}\right)<l_{0}$,

(iii) there exists a $J$-bilipschitz diffeomorphism

$$
\phi: \widehat{M}-\bigcup_{i=1}^{n} \mathbb{T}_{\epsilon}\left(T_{i}\right) \rightarrow M-\bigcup_{i=1}^{n} \mathbb{T}_{\epsilon}\left(\gamma_{i}\right) .
$$


Proof If the filled manifold $M$ exists, the drilling theorem says that there exists some $l_{0}^{\prime}$, depending only on $J$ and $\epsilon$, such that if $\sum_{i=1}^{n} l\left(\gamma_{i}\right)<l_{0}^{\prime}$, then there is a $J$-bilipschitz diffeomorphism

$$
\phi: \widehat{M}-\bigcup_{i=1}^{n} \mathbb{T}_{\epsilon}\left(T_{i}\right) \rightarrow M-\bigcup_{i=1}^{n} \mathbb{T}_{\epsilon}\left(\gamma_{i}\right) .
$$

Let $\ell=\min \left\{l_{0}, l_{0}^{\prime}\right\}$ where $l_{0}$ is the constant given in the statement of the Corollary. We will show there exists a $K$ such that if the normalized length of $\beta_{i}$ is at least $K$ for $1 \leq i \leq n$, then the filled manifold $M$ exists, and the length of $\gamma_{i}$ in $M$ is less than $\ell / n$.

We want to fill the cusps one at a time. Let $M^{0}=\widehat{M}$, and if it exists let $M^{i}$ be the $\beta_{i}$ filling of $M^{i-1}$. We will use the notation $l_{M^{i}}\left(\gamma_{j}\right)$ to denote the length of $\gamma_{j}$ in $M^{i}$. In this notation, we want to show that $M^{n}=M$ exists and that $l_{M^{n}}\left(\gamma_{i}\right)<\ell / n$ for all $1 \leq i \leq n$.

By the filling theorem, there exists some $K^{\prime}$ (independent of $i$ ) such that if the normalized length of $\beta_{i}$ in $M^{i-1}$ is at least $K^{\prime}$ then we have the following:

- The $\beta_{i}$-filling of $M^{i-1}$, which we will call $M^{i}$, exists.

- The length of $\gamma_{i}$ in $M^{i}$ satisfies $l_{M^{i}}\left(\gamma_{i}\right)<\ell /\left(n 2^{n}\right)$.

- There is a 2-bilipschitz map $\phi_{i}: M^{i-1}-\mathbb{T}_{\epsilon^{\prime}}\left(T_{i}\right) \rightarrow M^{i}-\mathbb{T}_{\epsilon^{\prime}}\left(\gamma_{i}\right)$ for some $\epsilon_{3} \geq \epsilon^{\prime}>0$.

Now let $K>4^{n} K^{\prime}$. If the normalized length of $\beta_{1}$ is at least $K$, we can do the first filling to obtain $M^{1}$. We now apply the filling theorem inductively. In the $i$-th filling, the length of $\gamma_{j}$ (for any $1 \leq j<i$ ) does not increase by more than factor of 2 since $\phi_{i}$ is 2 -bilipschitz. We next claim that the normalized length of $\beta_{j}$ (for any $i<j \leq n$ ) does not decrease by more than factor of 4 .

Fix a torus cross-section $T=T^{2} \times\{1\}$ of the cusp $T_{j} \cong T^{2} \times[0, \infty)$ contained in $M^{i-1}-\mathbb{T}_{\epsilon}\left(T_{i}\right)$. Let $\mu$ be a curve on $T$ homotopic to the meridian $\beta_{j}$. The normalized length of $\beta_{j}$ in $M^{i-1}$ is $l(\mu) / \sqrt{\operatorname{Area}(T)}$, where $l(\mu)$ is the length of a geodesic representative of $\mu$ on $T$ with respect to the induced Euclidean metric on $T$. Since $\phi_{i}$ is 2-bilipschitz, the length of a geodesic representative of $\phi_{i}(\mu)$ on $\phi_{i}(T)$ is bounded by $l\left(\phi_{i}(\mu)\right)>l(\mu) / 2$, and also $\operatorname{Area}\left(\phi_{i}(T)\right)<4(\operatorname{Area}(T))$. Thus

$$
\frac{l\left(\phi_{i}(\mu)\right)}{\sqrt{\operatorname{Area}\left(\phi_{i}(T)\right)}}>\frac{l(\mu) / 2}{\sqrt{4(\operatorname{Area}(T))}} .
$$

By [11, Theorem 6.12] (see the remark following Theorem 4.1), we can assume $\phi_{i}(T)$ is a flat cross-section of the $j$-th cusp in $M^{i}$. Thus the ratio $l\left(\phi_{i}(\mu)\right) / \sqrt{\operatorname{Area}\left(\phi_{i}(T)\right)}$ in the left-hand side of the inequality above is the normalized length of $\beta_{j}$ in $M^{i}$. 
This completes the proof that the normalized length of $\beta_{j}$ does not shrink by more than a factor of 4 during the $i$-th filling.

Thus, for any $1 \leq i \leq n$, the normalized length of $\beta_{i}$ in $M^{i-1}$ is at least $4^{n-i} K^{\prime}$. So we can apply the filling theorem $n$ times to get $M$, the $\bigcup \beta_{i}$-filling of $\widehat{M}$. This completes part (i). Since the length of $\gamma_{i}$ in $M^{i}$ is less than $\ell /\left(n 2^{n}\right)$, the length of $\gamma_{i}$ in $M$ is less than $(\ell / n)\left(2^{n-i} / 2^{n}\right) \leq \ell / n$. Hence $\sum_{i=1}^{n} l\left(\gamma_{i}\right)<\ell$. Since $\ell=\min \left\{l_{0}, l_{0}^{\prime}\right\}$ this completes parts (ii) and (iii).

Now suppose that $\widehat{M}$ is the $\gamma$-drilling of $M$, and let $T$ denote the new cusp of $\widehat{M}$. Recall that if $\gamma$ is sufficiently short, the drilling theorem provides a bilipschitz diffeomorphism $\phi: \widehat{M}-\mathbb{T}_{\epsilon_{3}}(T) \rightarrow M-\mathbb{T}_{\epsilon_{3}}(\gamma)$. There is a unique slope $\beta$ on $\partial \mathbb{T}_{\epsilon_{3}}(T)$ such that $\phi(\beta)$ bounds a disk in $\mathbb{T}_{\epsilon_{3}}(\gamma) \subset M$, but $\beta$ does not bound a disk in $\widehat{M}$. Equivalently, the $\beta$-filling of $\widehat{M}$ (if it exists) is $M$. We say that $\beta$ is the meridian of $\widehat{M}$. If $\gamma$ is sufficiently short, then one can bound the normalized length of $\beta$ in $\widehat{M}$ from below. This is stated without proof of [20, Theorem 2.4(2)].

Proposition 4.14 Let $K>0$. There exists $l_{0}>0$ such that the following holds. Let $M$ be a geometrically finite manifold containing a geodesic $\gamma$ of length less than $l_{0}$. If $\widehat{M}$ is the $\gamma$-drilling of $M$ and $\beta$ is the meridian in $\widehat{M}$ that bounds a disk in $M$, then the normalized length $L$ of the meridian $\beta$ in $\widehat{M}$ is at least $K$.

Proof By the drilling theorem, there exists $l_{1}$ such that if $l(\gamma)<l_{1}$ then there exists a 2-bilipschitz map

$$
\phi: \widehat{M}-\mathbb{T}_{\epsilon_{3}}(T) \rightarrow M-\mathbb{T}_{\epsilon_{3}}(\gamma) .
$$

Suppose that $R$ is the distance between $\gamma$ and $\partial \mathbb{T}_{\epsilon_{3}}(\gamma)$. The area of the boundary of this Margulis tube in $M$ is equal to

$$
A\left(\partial \mathbb{T}_{\epsilon_{3}}(\gamma)\right)=A=2 \pi l(\gamma) \sinh (R) \cosh (R) .
$$

(For example, see [34, page 403].) Here $2 \pi \sinh (R)$ gives the length of the meridian on $\partial \mathbb{T}_{\epsilon_{3}}(\gamma)$ in $M$. Define the normalized length of $\beta$ in $M$ to be $L_{M}(\beta)=$ $2 \pi \sinh (R) / \sqrt{A}$. This is the length of (a geodesic representative of) $\phi(\beta)$ on $\partial \mathbb{T}_{\epsilon_{3}}(\gamma)$ divided by the square root of the area of $\partial \mathbb{T}_{\epsilon_{3}}(\gamma)$. Unlike the normalized length of $\beta$ in $\widehat{M}$, which is the length of $\beta$ on $\partial \mathbb{T}_{\epsilon_{3}}(T)$ divided by the square root of the area of $\partial \mathbb{T}_{\epsilon_{3}}(T)$, the normalized length of $\beta$ in $M$ depends on the size of the Margulis 
tube (in this case $\epsilon_{3}$ ).

Now

$$
\begin{aligned}
& L_{M}(\beta)=\frac{2 \pi \sinh (R)}{\sqrt{A}}=\frac{\sqrt{A}}{l(\gamma) \cosh (R)} . \\
& L_{M}(\beta)=\sqrt{\frac{2 \pi \tanh (R)}{l(\gamma)}} .
\end{aligned}
$$

Thus

The estimates in Brooks and Matelski [23] imply that given any $R_{0}$, there is some $l_{2}^{\prime}$ such that if $l(\gamma)<l_{2}^{\prime}$ then $R>R_{0}$. Hence, there is some $l_{2}$ such that if $l(\gamma)<l_{2}$, then $L_{M}(\beta)>4 K$.

Now let $l_{0}=\min \left\{l_{1}, l_{2}\right\}$. This implies the filling map restricts to a 2 -bilipschitz diffeomorphism on the boundary tori: $\phi^{-1}: \partial \mathbb{T}_{\epsilon_{3}}(\gamma) \rightarrow \partial \mathbb{T}_{\epsilon_{3}}(T)$. Hence, as we saw in the proof of the previous corollary, the normalized length of $\beta$ in $\widehat{M}$ is no less than $\frac{1}{4}$ times the normalized length of $\beta$ on $\partial \mathbb{T}_{\epsilon_{3}}(\gamma)$. Thus, the normalized length of the meridian in $\widehat{M}$ (which we are simply denoting by $L$ ) is at least

$$
L \geq \frac{1}{4} L_{M}(\beta)>K .
$$

Remark One can also prove Proposition 4.14 using the tools developed in the proofs of Proposition 4.4, Lemma 4.5. One defines a one-parameter family of cone-manifolds by decreasing the cone-angle about $\gamma$ from $2 \pi$ to 0 , showing that the maximal radius of a neighborhood of $\gamma$ does not become too small. There is some $l_{1}$ such that if the length of $\gamma$ is less than $l_{1}$ then the one-parameter family of cone-manifolds can be defined for all $t \in\left[0,(2 \pi)^{2}\right]$, and the estimate

$$
\left|\frac{d u}{d t}\right| \leq 4
$$

can be applied for all $t$. Recall that $u(t) \rightarrow L^{2}$ as $t \rightarrow 0$ where $L^{2}$ is the square of the normalized length of $\beta$ in $\widehat{M}$, and $u\left((2 \pi)^{2}\right)=2 \pi / l(\gamma)$ when $t=(2 \pi)^{2}$. From this, one can see that given any $K$, there exists some $l_{0}$ such that if $l(\gamma)<l_{0}$ then $L^{2}>K^{2}$.

\section{Constructing a local model of the deformation space}

Let $S$ be a closed surface of genus at least two, set $N=S \times I$, and define the paring locus $P \subset \partial N$ to be a collection of annuli forming a pants decomposition of $S \times\{1\}$. We will define a space $\mathcal{A}$ and show that $\mathcal{A}$ locally models a dense subset of the deformation space $A H(N)$. We do this by constructing a map $\Phi: \mathcal{A} \rightarrow M P(N) \cup M P(N, P)$, 
and showing that there is some open set $U \subset \mathcal{A}$, a point $\sigma$ in $\operatorname{MP}(N, P)$, and a neighborhood $V$ of $\sigma$ in $M P(N) \cup M P(N, P)$ such that $\left.\Phi\right|_{U}: U \rightarrow V$ is a homeomorphism. The definition of $\Phi$ and the proof that it is continuous is in Section 5.5. Then, in Section 5.6 and Section 5.7, we show that $\Phi$ is a local homeomorphism to $M P(N) \cup M P(N, P) \subset A H(N)$. In order to find a point where $\operatorname{MP}(N) \cup M P(N, P)$ is not locally connected, we must first show there exists such a point in $\mathcal{A}$.

We begin by studying lower dimensional analogues to $\mathcal{A}$. Let $S_{1,1}$ and $S_{0,4}$ denote the punctured torus and four-punctured sphere respectively. In Section 5.1, we define spaces $\mathcal{A}_{1,1}$ and $\mathcal{A}_{0,4}$ which, by Bromberg's results [20], locally model the deformation spaces $A H\left(S_{1,1} \times I, \partial S_{1,1} \times I\right)$ and $A H\left(S_{0,4} \times I, \partial S_{0,4} \times I\right)$ respectively. We define $\mathcal{A}$ in Section 5.2 similarly, and relate $\mathcal{A}$ to the lower dimensional versions by showing there exists a continuous surjection $\Pi: \mathcal{A} \rightarrow \mathcal{A}_{1,1}$ in Section 5.3. We use this in Section 5.4, along with the fact that $\mathcal{A}_{1,1}$ is not locally connected [20], to show that $\mathcal{A}$ is not locally connected. In fact, in Section 5.4, we find a point of $\mathcal{A}$ with the property that any sufficiently small neighborhood of this point contains infinitely many components that are bounded apart from each other. In Section 6, we will use this description of the components of a neighborhood $U \subset \mathcal{A}$ and the filling theorem, which is used in the definition of $\Phi$, to show that there is a point $\sigma_{0} \in M P(N, P)$ such that for any sufficiently small neighborhood $\sigma_{0} \in V \subset M P(N) \cup M P(N, P)$, the closure of $V$ has infinitely many components. Along with the Density Theorem, this will be used to show that $A H(N)$ is not locally connected.

\subsection{The punctured torus and four-punctured sphere}

Define $N_{1,1}=S_{1,1} \times I$ and $P_{1,1}=\partial S_{1,1} \times I$. Let $P_{1,1}^{\prime}$ be the union of $P_{1,1}$ with a nonperipheral annulus in $S_{1,1} \times\{1\}$ about a curve $b \times\{1\}$ (see Figure 2). Let $\hat{N}_{1,1}$ be the manifold obtained by removing an open tubular neighborhood of $b \times\left\{\frac{1}{2}\right\}$ from $N_{1,1}$, and let $\widehat{P}_{1,1}$ be the union of $P_{1,1}$ with the toroidal boundary component of $\hat{N}_{1,1}$.
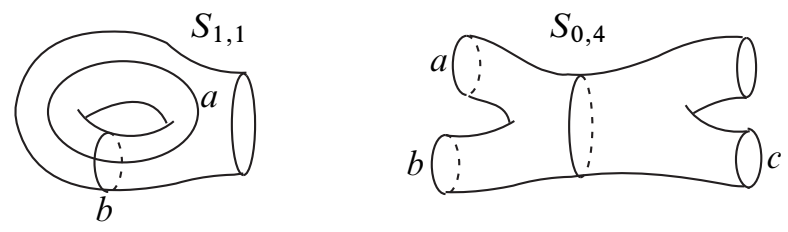

Figure 2: Orient the curves $a, b$ on $S_{1,1}$ and identify a presentation $\pi_{1}\left(S_{1,1}\right)=\langle a, b\rangle$. Similarly, orient $a, b, c$ on $S_{0,4}$ and identify a presentation $\pi_{1}\left(S_{0,4}\right)=\langle a, b, c\rangle$.

Recall from Section 2 that the components of $M P\left(N_{1,1}, P_{1,1}^{\prime}\right)$ are in one-to-one correspondence with the marked pared homeomorphism types of manifolds pared 
homotopy equivalent to $\left(N_{1,1}, P_{1,1}^{\prime}\right)$. Fix an orientation on $N_{1,1}$ and let $\bar{N}_{1,1}$ denote $N_{1,1}$ with the opposite orientation. Then there are two components of $\operatorname{MP}\left(N_{1,1}, P_{1,1}^{\prime}\right)$ corresponding to $F^{-1}\left(\left[\left(N_{1,1}, P_{1,1}^{\prime}\right), \mathrm{id}\right]\right)$ and $F^{-1}\left(\left[\left(\bar{N}_{1,1}, P_{1,1}^{\prime}\right)\right.\right.$, id $\left.]\right)$. We denote the former by $M P_{0}\left(N_{1,1}, P_{1,1}^{\prime}\right)$.

Given any $z \in \mathbb{C}$, one can define a representation $\sigma_{z}: \pi_{1}\left(N_{1,1}\right) \rightarrow \operatorname{PSL}(2, \mathbb{C})$ by

$$
\sigma_{z}(a)=\left(\begin{array}{cc}
i z & i \\
i & 0
\end{array}\right), \quad \sigma_{z}(b)=\left(\begin{array}{ll}
1 & 2 \\
0 & 1
\end{array}\right)
$$

For any $\sigma \in M P\left(N_{1,1}, P_{1,1}^{\prime}\right)$ there is a unique $z$ such that $\sigma_{z}$ is in the conjugacy class of $\sigma$ (see [43, Section 6.3] and [37]). This defines an embedding of $M P\left(N_{1,1}, P_{1,1}^{\prime}\right)$ into $\mathbb{C}$. Let $\mathcal{M}$ denote the image of this embedding. That is,

$$
\mathcal{M}=\left\{z \in \mathbb{C}: \sigma_{z} \in M P\left(N_{1,1}, P_{1,1}^{\prime}\right)\right\} .
$$

One component of this is called the Maskit slice, $\mathcal{M}^{+}$, and denotes the set of all $z \in \mathbb{C}$ such that $\sigma_{z} \in M P_{0}\left(N_{1,1}, P_{1,1}^{\prime}\right)$. The following lemma, due to Keen and Series [37] and Wright [74], summarizes the basic properties of the Maskit slice we will need (see also [20, Proposition 4.4]).

Lemma 5.1 The set $\mathcal{M}$ has two components: one component, $\mathcal{M}^{+}$, is contained in the upper half plane. Its boundary lies in the horizontal strip $\{z \mid 1 \leq \operatorname{Im}(z) \leq 2\}$ but is not a horizontal line. The other component, $\mathcal{M}^{-}$, is the mirror image in the sense that $z \in \mathcal{M}^{ \pm}$if and only if $-z \in \mathcal{M}^{\mp}$. Also, the Maskit slice is invariant under the horizontal translation $z \mapsto z+2$.

Remark We are following the Keen-Series convention by defining $\mathcal{M}^{+}$and $\mathcal{M}^{-}$to be open sets, whereas Bromberg uses $\mathcal{M}^{ \pm}$to denote the closures of these sets in [20, Section 4]. The set $\mathcal{M}^{+}$is also known as the Maskit embedding of the Teichmüller space of punctured tori.

Given $w \in \mathbb{C}$ and a conjugacy class of representations $\sigma \in M P_{0}\left(N_{1,1}, P_{1,1}^{\prime}\right)$, one can define a representation $\sigma_{z, w}$ of $\pi_{1}\left(\hat{N}_{1,1}\right)=\langle a, b, c \mid[b, c]=1\rangle$ in the following way. There is a unique $z \in \mathcal{M}^{+}$such that the representation $\sigma_{z}$ represents the conjugacy class $\sigma$. Define

$$
\sigma_{z, w}(a)=\sigma_{z}(a), \quad \sigma_{z, w}(b)=\sigma_{z}(b), \quad \sigma_{z, w}(c)=\left(\begin{array}{cc}
1 & w \\
0 & 1
\end{array}\right) .
$$

Note that $\sigma_{z, w}$ is an actual representation of $\pi_{1}\left(\widehat{N}_{1,1}\right)$, but we will also use $\sigma_{z, w}$ to refer to the conjugacy class. 
Define $\mathcal{A}_{1,1}$ to be

$$
\begin{aligned}
\mathcal{A}_{1,1}=\left\{(\sigma, w) \in M P_{0}\left(N_{1,1}, P_{1,1}^{\prime}\right) \times \widehat{\mathbb{C}}: w\right. & =\infty, \text { or } \\
& \left.\sigma_{z, w} \in M P\left(\widehat{N}_{1,1}, \widehat{P}_{1,1}\right) \text { and } \operatorname{Im}(w)>0\right\} .
\end{aligned}
$$

Note that this is what Bromberg calls $\mathcal{A}$ in [20].

One defines $\mathcal{A}_{0,4}$ similarly. Let $N_{0,4}=S_{0,4} \times I$, let $P_{0,4}=\partial S_{0,4} \times I$, and define $P_{0,4}^{\prime}$ to be the union of $P_{0,4}$ with a nonperipheral annulus in $S_{0,4} \times\{1\}$ whose core curve is $a b \times\{1\}$ (see Figure 2). Let $\widehat{N}_{0,4}$ be the manifold obtained by removing an open tubular neighborhood of $a b \times\left\{\frac{1}{2}\right\}$ from $N_{0,4}$, and define $\widehat{P}_{0,4}$ to be the union of $P_{0,4}$ with the toroidal boundary component of $\hat{N}_{0,4}$.

Given any $\sigma \in M P\left(N_{0,4}, P_{0,4}^{\prime}\right)$, there is a unique $z \in \mathbb{C}$ such that the representation $\sigma_{z}: \pi_{1}\left(N_{0,4}\right) \rightarrow \operatorname{PSL}(2, \mathbb{C})$ defined by

$$
\sigma_{z}(a)=\left(\begin{array}{ll}
-3 & 2 \\
-2 & 1
\end{array}\right), \quad \sigma_{z}(b)=\left(\begin{array}{ll}
1 & 0 \\
2 & 1
\end{array}\right), \quad \sigma_{z}(c)=\left(\begin{array}{cc}
-1+2 z & -2 z^{2} \\
2 & -1-2 z
\end{array}\right)
$$

represents the conjugacy class of $\sigma$ [43, Section 6.1]. Note that for any $z$, one can check that

$$
\sigma_{z}(a b)=\left(\begin{array}{ll}
1 & 2 \\
0 & 1
\end{array}\right)
$$

The Maskit slice for $S_{0,4}$, denoted $\mathcal{M}_{0,4}^{+}$, is the set of all $z \in \mathbb{C}$ such that $\sigma_{z} \in$ $M P_{0}\left(N_{0,4}, P_{0,4}^{\prime}\right)$, and its mirror image in the lower half plane will be denoted by $\mathcal{M}_{0,4}^{-}$. Again, we let $\mathcal{M}_{0,4}=\mathcal{M}_{0,4}^{+} \cup \mathcal{M}_{0,4}^{-}$. Kra shows that $z \in \mathcal{M}_{0,4}$ if and only if $2 z \in \mathcal{M}^{+}[43$, page 558].

Given $w \in \mathbb{C}$ and a conjugacy class of representations $\sigma \in M P_{0}\left(N_{0,4}, P_{0,4}^{\prime}\right)$, one can define a representation $\sigma_{z, w}$ of $\pi_{1}\left(\hat{N}_{0,4}\right)=\langle a, b, c, d \mid[a b, d]=1\rangle$ in the following way. There is a unique $z \in \mathcal{M}_{0,4}^{+}$such that the representation $\sigma_{z}$ represents the conjugacy class $\sigma$. Define

$$
\sigma_{z, w}(a)=\sigma_{z}(a), \quad \sigma_{z, w}(b)=\sigma_{z}(b), \quad \sigma_{z, w}(c)=\sigma_{z}(c), \quad \sigma_{z, w}(d)=\left(\begin{array}{ll}
1 & w \\
0 & 1
\end{array}\right) .
$$

Define $\mathcal{A}_{0,4}$ to be

$$
\begin{aligned}
\mathcal{A}_{0,4}=\left\{(\sigma, w) \in M P_{0}\left(N_{0,4}, P_{0,4}^{\prime}\right) \times \widehat{\mathbb{C}}: w\right. & =\infty, \text { or } \\
& \left.\sigma_{z, w} \in M P\left(\widehat{N}_{0,4}, \widehat{P}_{0,4}\right) \text { and } \operatorname{Im}(w)>0\right\} .
\end{aligned}
$$

Given $\sigma \in M P_{0}\left(N_{1,1}, P_{1,1}^{\prime}\right)$ and $w \in \mathbb{C}$, Bromberg characterizes when $(\sigma, w) \in \mathcal{A}_{1,1}$. 
Lemma 5.2 (i) (Bromberg [20]) Let $\sigma_{z} \in M P_{0}\left(N_{1,1}, P_{1,1}^{\prime}\right)$ and $w \in \mathbb{C}$ with $\operatorname{Im}(w)>0$. Then $\sigma_{z, w} \in M P\left(\widehat{N}_{1,1}, \widehat{P}_{1,1}\right)$ if and only if there exists an integer $n$ such that $z-n w \in \mathcal{M}^{+}$and $z-(n+1) w \in \mathcal{M}^{-}$.

(ii) Let $\sigma_{z} \in M P_{0}\left(N_{0,4}, P_{0,4}^{\prime}\right)$ and $w \in \mathbb{C}$ with $\operatorname{Im}(w)>0$. Then $\sigma_{z, w} \in M P\left(\hat{N}_{0,4}, \hat{P}_{0,4}\right)$ if and only if there exists an integer $n$ such that $z-n w \in \mathcal{M}_{0,4}^{+}$and $z-(n+1) w \in \mathcal{M}_{0,4}^{-}$.

Remark Part (i) is [20, Proposition 4.7]. The proof of (ii) is nearly identical to that of (i), and we refer the reader to [47, Lemma 5.1] for the necessary adaptations.

Although in the definitions of $\mathcal{A}_{1,1}$ and $\mathcal{A}_{0,4}$ we only require $\operatorname{Im}(w)>0$, the following lemma allows us to give a positive lower bound. This will be used to obtain Corollary 5.6 which is used in the proof of Lemma 5.13.

Lemma 5.3 If $(\sigma, w) \in \mathcal{A}_{1,1}$, then $w=\infty$ or $\operatorname{Im}(w)>2$. If $(\sigma, w) \in \mathcal{A}_{0,4}$ then $w=\infty$ or $\operatorname{Im}(w)>1$.

Proof If $(\sigma, w) \in \mathcal{A}_{1,1}$, then there is some $z$ such that the conjugacy class of $\sigma_{z}$ is $\sigma$. If $w \neq \infty$, then by Lemma 5.2(i) there is some integer $n$ such that $z-n w \in \mathcal{M}^{+}$and $z-(n+1) w \in \mathcal{M}^{-}$. Thus $\operatorname{Im}(w)$ is at least as large as the vertical distance between the components of $\mathcal{M}$. Wright shows that if $\operatorname{Im}(z) \leq 1$ then $z \notin \mathcal{M}^{+}$[74] (see also [43, page 534, 558] and the comments after [37, Proposition 2.6]). Since $\zeta \in \mathcal{M}^{-}$if and only if $-\zeta \in \mathcal{M}^{+}$, the distance between these two components of the Maskit slice is at least 2. It follows that if $(\sigma, w) \in \mathcal{A}_{1,1}$ and $w \neq \infty$ then $\operatorname{Im}(w)>2$.

The four-punctured sphere case is similar. Suppose $(\sigma, w) \in \mathcal{A}_{0,4}$ and $w \neq \infty$. Let $z$ be such that the conjugacy class of $\sigma_{z}$ is $\sigma$. By Lemma 5.2(ii), $\operatorname{Im}(w)$ is at least the vertical distance between the two components of $\mathcal{M}_{0,4}$. Since $\zeta \in \mathcal{M}_{0,4}$ if and only if $2 \zeta \in \mathcal{M}$ [43, page 558], we must have $\operatorname{Im}(w)>1$.

\subsection{The model space $\mathcal{A}$}

For surfaces with higher dimensional Teichmüller spaces, the construction of $\mathcal{A}$ takes more bookkeeping but is otherwise similar to $\mathcal{A}_{1,1}$ and $\mathcal{A}_{0,4}$.

Recall $N=S \times I$. Let $\left\{\gamma_{i}\right\}_{i=1}^{d}$ be a pants decomposition of $S$ (recall from Section 1 that we will abbreviate $d=3 g-3$ ). Although fixing any pants decomposition would be acceptable, we will make some choices that make it more convenient to apply results of [20]. We will choose $\gamma_{2}$ to be a curve that separates $S$ into a punctured torus and a punctured genus $g-1$ surface, and let $\gamma_{1}$ be a curve in the punctured torus 


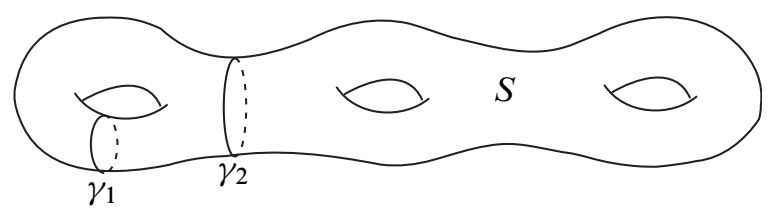

Figure 3: Part of the pants decomposition that we will fix throughout the rest of the argument

component of $S-\gamma_{2}$. Also, fix an orientation on each $\gamma_{i}$ to distinguish $\gamma_{i}$ from its inverse in $\pi_{1}(S)$.

For each $i$, define a homeomorphism $G_{i}$ from either $S_{1,1}$ or $S_{0,4}$ to the component of $\left(S-\bigcup_{j \neq i} \gamma_{j}\right)$ containing $\gamma_{i}$. Moreover, using the markings of $S_{1,1}$ and $S_{0,4}$ from Figure 2 , define $G_{i}$ on $S_{1,1}$ so that $\left(G_{i}\right)_{*}(b)=\gamma_{i}$ and define $G_{i}$ on $S_{0,4}$ so that $\left(G_{i}\right)_{*}(a b)=\gamma_{i}$.

Let $P$ be a collection of $d$ disjoint annuli in $S \times\{1\}$ such that $\gamma_{i} \times\{1\}$ is core curve of the $i$-th annulus of $P$ (see Figure 3). Then $M P(N, P)$ has $2^{d}$ components corresponding to whether $\gamma_{i}$ is parabolic to one side or the other. For two of these components, all of the parabolics will be on the same side of $N$. In other words, for any $\rho$ in one of these components, $M_{\rho}$ will have exactly one closed component of its conformal boundary homeomorphic to $S$. Using the notation from Section 2, these two components can be identified with $F^{-1}[(N, P)$, id $]$ and $F^{-1}[(\bar{N}, P)$, id] where $\bar{N}$ denotes $N$ with the opposite orientation. Label the former component by $M P_{0}(N, P)$. These will be the marked hyperbolic manifolds with a rank-1 cusp associated to each $\gamma_{i}$ such that the cusps all occur to the "top" of the manifold.

We now elaborate on what we mean by the "top" of a hyperbolic manifold. This discussion will be useful in distinguishing whether or not a point $\rho \in M P(N, P)$ lies in $M P_{0}(N, P)$. Given $\rho \in M P(N, P)$, there exists an embedding $f: S \rightarrow M_{\rho}=$ $\mathbb{H}^{3} / \rho\left(\pi_{1}(N)\right)$ such that $f_{*}=\rho$. The orientation on $S$ induces an orientation on $f(S)$. This orientation, together with a normal direction to $f(S)$, defines an orientation on $M_{\rho}$, and for only one of the two normal directions will this orientation be compatible with the orientation induced on $M_{\rho}$ as a quotient of $\mathbb{H}^{3}$. This distinguishes a top side of $f(S)$, and we say the top of the manifold $M_{\rho}$ with respect to $f(S)$ is the component of $M_{\rho}-f(S)$ that lies to the top side of $f(S)$. If $\rho \in M P(N, P)$, then there are $d$ rank-1 cusps associated to each of the components of $P$. If each of these cusps lies in the top of $M_{\rho}-f(S)$, then we say $\rho \in M P_{0}(N, P)$. Since any two embeddings $f: S \rightarrow M_{\rho}$ such that $f_{*}=\rho$ are isotopic [73], this notion is independent of the map $f$. Likewise, if $\rho \in M P(N, P)$ and $X$ is a conformal boundary component of $M_{\rho}$, then we can distinguish whether $X$ lies on the top or bottom side of $M_{\rho}$. 
Let $\widehat{N}$ be obtained by drilling a set of $d$ curves out of $N$. Specifically, let $\gamma_{i} \times\{1 / 2\}$ be a collection of $d$ curves in $S \times\{1 / 2\}$. Let $U_{i}$ be an open collar neighborhood of $\gamma_{i} \times\{1 / 2\}$ such that the elements of the collection $\bigcup U_{i}$ are pairwise disjoint. Let

$$
\widehat{N}=N-\bigcup_{i=1}^{d} U_{i} .
$$

Define $\widehat{P}=\bigcup \partial U_{i}$. Note that $\pi_{1}(\hat{N})$ is generated by $\pi_{1}(N)$ and $d$ new elements $\beta_{i}$ corresponding to meridians of $\partial U_{i}$. Since the meridian of $\partial U_{i}$ commutes with any longitude of $\partial U_{i}$, there are new relations. We will use $\gamma_{i}$ to denote both a curve and the element of $\pi_{1}(N)$ corresponding to that curve. Then $\left[\beta_{i}, \gamma_{i}\right]=1$. We write

$$
\pi_{1}(\widehat{N})=\left\langle\pi_{1}(N), \beta_{1}, \ldots, \beta_{d} \mid\left[\beta_{i}, \gamma_{i}\right]=1\right\rangle
$$

with the understanding that $\pi_{1}(N)$ has generators besides $\gamma_{i}$ and some of its own relations.

Given $\sigma \in M P_{0}(N, P)$ and $w=\left(w_{1}, \ldots, w_{d}\right) \in \mathbb{C}^{d}$, we describe a process for constructing a representation $\sigma_{w}: \pi_{1}(\hat{N}) \rightarrow \operatorname{PSL}(2, \mathbb{C})$. We can find a representative in the conjugacy class determined by $\sigma$ (which by an abuse of notation, we still refer to as $\sigma$ ) such that

$$
\begin{aligned}
& \sigma\left(\gamma_{1}\right)=\left(\begin{array}{ll}
1 & 2 \\
0 & 1
\end{array}\right), \\
& \sigma\left(\gamma_{2}\right)=\left(\begin{array}{cc}
-3 & -2 \\
2 & 1
\end{array}\right) .
\end{aligned}
$$

We choose these matrices to parallel the construction of $\mathcal{A}_{1,1}$ in the previous section. Recall that we fixed some homeomorphism $G_{1}$ from $S_{1,1}$ to the subsurface of $S$ bounded by $\gamma_{2}$ that contains $\gamma_{1}$. There is a unique $z \in \mathcal{M}^{+}$such that $\sigma \circ\left(G_{1}\right)_{*}$ is conjugate to $\sigma_{z}$. Note that for any $z$,

$\sigma_{z}(b)=\left(\begin{array}{ll}1 & 2 \\ 0 & 1\end{array}\right)=\sigma \circ\left(G_{1}\right)_{*}(b), \sigma_{z}\left(b^{-1} a^{-1} b a\right)=\left(\begin{array}{cc}-3 & -2 \\ 2 & 1\end{array}\right)=\sigma \circ\left(G_{1}\right)_{*}\left(b^{-1} a^{-1} b a\right)$.

Thus, specifying $\sigma$ on $\gamma_{1}$ and $\gamma_{2}$ determines a well-defined representation in the conjugacy class of $\sigma$ that restricts to $\sigma_{z}$ for some $z$ on $G_{1}\left(S_{1,1}\right)$.

We define $\sigma_{w_{1}}: \pi_{1}\left(N-U_{1}\right) \rightarrow \operatorname{PSL}(2, \mathbb{C})$ by $\sigma_{w_{1}}(\alpha)=\sigma(\alpha)$ for all $\alpha \in \pi_{1}(N)$ and

$$
\sigma_{w_{1}}\left(\beta_{1}\right)=\left(\begin{array}{cc}
1 & w_{1} \\
0 & 1
\end{array}\right)
$$


We then inductively define $\sigma_{w_{1}, \ldots, w_{i}}: \pi_{1}\left(N-\bigcup_{j=1}^{i} U_{j}\right) \rightarrow \operatorname{PSL}(2, \mathbb{C})$ by conjugating $\sigma_{w_{1}, \ldots, w_{i-1}}$ so that

$$
\sigma_{w_{1}, \ldots, w_{i-1}}\left(\gamma_{i}\right)=\left(\begin{array}{ll}
1 & 2 \\
0 & 1
\end{array}\right)
$$

(there is some ambiguity here that will be clarified below). Then define $\sigma_{w_{1}, \ldots, w_{i}}$ by $\sigma_{w_{1}, \ldots, w_{i}}(\alpha)=\sigma_{w_{1}, \ldots, w_{i-1}}(\alpha)$ for all $\alpha \in \pi_{1}\left(N-\bigcup_{j=1}^{i-1} U_{j}\right)$, and

$$
\sigma_{w_{1}, \ldots, w_{i}}\left(\beta_{i}\right)=\left(\begin{array}{cc}
1 & w_{i} \\
0 & 1
\end{array}\right)
$$

As we indicated above, specifying that we should conjugate $\sigma_{w_{1}, \ldots, w_{i-1}}$ so that $\gamma_{i}$ is sent to

$$
\left(\begin{array}{ll}
1 & 2 \\
0 & 1
\end{array}\right)
$$

does not determine a unique representation, but we now show how a unique representation can be specified. Each curve $\gamma_{i}$ lies in either a four-punctured sphere or punctured torus component of

$$
S-\bigcup_{j \neq i} \gamma_{j}
$$

that we have marked by a homeomorphism $G_{i}$ from $S_{1,1}$ or $S_{0,4}$. If $\gamma_{i}$ lies in a punctured-torus component bounded by some curve $\gamma_{j}$ then we conjugate $\sigma_{w_{1}, \ldots, w_{i-1}}$ such that

$\sigma_{w_{1}, \ldots, w_{i-1}} \circ\left(G_{i}\right)_{*}(b)=\left(\begin{array}{ll}1 & 2 \\ 0 & 1\end{array}\right) \quad$ and $\quad \sigma_{w_{1}, \ldots, w_{i-1}} \circ\left(G_{i}\right)_{*}\left(b^{-1} a^{-1} b a\right)=\left(\begin{array}{cc}-3 & -2 \\ 2 & 1\end{array}\right)$.

Since $G_{i}$ was chosen so that $\left(G_{i}\right)_{*}(b)=\gamma_{i}$, this ensures

$$
\sigma_{w_{1}, \ldots, w_{i-1}}\left(\gamma_{i}\right)=\left(\begin{array}{ll}
1 & 2 \\
0 & 1
\end{array}\right)
$$

and the condition that

$$
\sigma_{w_{1}, \ldots, w_{i-1}}\left(\gamma_{j}\right)=\left(\begin{array}{cc}
-3 & -2 \\
2 & 1
\end{array}\right)
$$

specifies $\sigma_{w_{1}, \ldots, w_{i-1}}$ uniquely.

If $\gamma_{i}$ lies in a four-punctured sphere component, then we conjugate $\sigma_{w_{1}, \ldots, w_{i-1}}$ so that

$$
\sigma_{w_{1}, \ldots, w_{i-1}} \circ\left(G_{i}\right)_{*}(a)=\left(\begin{array}{ll}
-3 & 2 \\
-2 & 1
\end{array}\right) \quad \text { and } \quad \sigma_{w_{1}, \ldots, w_{i-1}} \circ\left(G_{i}\right)_{*}(b)=\left(\begin{array}{ll}
1 & 0 \\
2 & 1
\end{array}\right) .
$$

It follows that

$$
\sigma_{w_{1}, \ldots, w_{i-1}} \circ\left(G_{i}\right)_{*}(a b)=\left(\begin{array}{ll}
1 & 2 \\
0 & 1
\end{array}\right)
$$


After $d$ steps, we get a well-defined representation $\sigma_{w_{1}, \ldots, w_{d}}$ which we also denote by $\sigma_{w}: \pi_{1}(\hat{N}) \rightarrow \operatorname{PSL}(2, \mathbb{C})$. By construction, for each $i$ there exists some representation in the conjugacy class of $\sigma_{w}$ such that the generators $\gamma_{i}, \beta_{i}$ of $\pi_{1}\left(\partial U_{i}\right)$ are sent, respectively, to

$$
\left(\begin{array}{ll}
1 & 2 \\
0 & 1
\end{array}\right) \text { and }\left(\begin{array}{lc}
1 & w_{i} \\
0 & 1
\end{array}\right)
$$

Given $\sigma \in M P_{0}(N, P)$, not every choice of $w=\left(w_{1}, \ldots, w_{d}\right) \in \mathbb{C}^{d}$ will result in $\sigma_{w} \in M P(\widehat{N}, \widehat{P})$. Thus we are led to the following definition:

$$
\begin{aligned}
\mathcal{A}=\left\{(\sigma, w) \in M P_{0}(N, P) \times \widehat{\mathbb{C}}^{d}: w=(\infty, \ldots, \infty),\right. \text { or } \\
\left.\qquad \operatorname{Im}\left(w_{i}\right)>0 \text { and } \sigma_{w} \in \operatorname{MP}(\widehat{N}, \widehat{P})\right\} .
\end{aligned}
$$

\subsection{Projections of $\mathcal{A}$ to $\mathcal{A}_{1,1}$ and $\mathcal{A}_{0,4}$}

Now that we have defined the model space $\mathcal{A}$, we want to use the fact that $\mathcal{A}_{1,1}$ is not locally connected [20] to show that $\mathcal{A}$ is not locally connected. In this section, we show there is a continuous surjection $\Pi: \mathcal{A} \rightarrow \mathcal{A}_{1,1}$, and in the sequel we explain how this can be used to relate the local connectivity of $\mathcal{A}$ and $\mathcal{A}_{1,1}$.

By our choice of pants decomposition (see Figure 3), the annulus $\gamma_{2} \times[0,1]$ cuts $N$ into two pieces, one of which is homeomorphic to $N_{1,1}$ (and so we will refer to this component as $\left.N_{1,1}\right)$. Given $\sigma: \pi_{1}(N) \rightarrow \operatorname{PSL}(2, \mathbb{C})$, the restriction of $\sigma$ to this punctured torus defines a representation $\left.\sigma\right|_{\pi_{1}\left(N_{1,1}\right)}: \pi_{1}\left(N_{1,1}\right) \rightarrow \operatorname{PSL}(2, \mathbb{C})$.

Lemma 5.4 If $\sigma \in M P_{0}(N, P)$, then $\left.\sigma\right|_{\pi_{1}\left(N_{1,1}\right)} \in M P_{0}\left(N_{1,1}, P_{1,1}^{\prime}\right)$.

Proof Thurston showed that finitely generated subgroups of geometrically finite Kleinian groups with nonempty domain of discontinuity are geometrically finite [62, Proposition 7.1]. Since $P_{1,1}^{\prime}=P \cap N_{1,1}$, if $\sigma$ is minimally parabolic with respect to $P$, then $\left.\sigma\right|_{\pi_{1}\left(N_{1,1}\right)}$ is minimally parabolic with respect to $P_{1,1}^{\prime}$. Thus, $\left.\sigma\right|_{\pi_{1}\left(N_{1,1}\right)} \in$ $\operatorname{MP}\left(N_{1,1}, P_{1,1}^{\prime}\right)$.

Let $f: S \rightarrow \mathbb{H}^{3} / \sigma\left(\pi_{1}(N)\right)$ be any embedding that satisfies $f_{*}=\sigma$. Since $\sigma \in$ $M P_{0}(N, P)$, all of the cusps lie to the top of the image of this embedding. The manifold $\mathbb{H}^{3} /\left.\sigma\right|_{\pi_{1}\left(N_{1,1}\right)}\left(\pi_{1}\left(N_{1,1}\right)\right)$ covers $\mathbb{H}^{3} / \sigma\left(\pi_{1}(N)\right)$, and the restriction of $f$ to the punctured torus $S_{1,1}$ containing $\gamma_{1}$ lifts to an embedding $\left.f\right|_{S_{1,1}}: S_{1,1} \rightarrow$ $\mathbb{H}^{3} /\left.\sigma\right|_{\pi_{1}\left(N_{1,1}\right)}\left(\pi_{1}\left(N_{1,1}\right)\right)$ such that $f_{*}=\left.\sigma\right|_{\pi_{1}\left(N_{1,1}\right)}$. The cusp corresponding to $\gamma_{1}$ must lie above this embedding; hence, $\left.\sigma\right|_{\pi_{1}\left(N_{1,1}\right)} \in M P_{0}\left(N_{1,1}, P_{1,1}^{\prime}\right)$.

Lemma 5.4 allows us to define the projection map in the following lemma. 
Lemma 5.5 The map $\Pi: \mathcal{A} \rightarrow M P_{0}\left(N_{1,1}, P_{1,1}^{\prime}\right) \times \widehat{\mathbb{C}}$ defined by

$$
\Pi\left(\sigma, w_{1}, \ldots, w_{d}\right)=\left(\left.\sigma\right|_{\pi_{1}\left(N_{1,1}\right)}, w_{1}\right)
$$

is a continuous map such that $\Pi(\mathcal{A})=\mathcal{A}_{1,1}$.

Proof We first claim $\Pi(\mathcal{A}) \subset \mathcal{A}_{1,1}$. Recall the definitions of $\mathcal{A}$ and $\mathcal{A}_{1,1}$. If a point $\left(\sigma, w_{1}, \ldots, w_{d}\right) \in \mathcal{A}$ satisfies $\left(w_{1}, \ldots, w_{d}\right) \neq(\infty, \ldots, \infty)$ then the extension $\sigma_{w_{1}, \ldots, w_{d}} \in \operatorname{MP}(\hat{N}, \widehat{P})$. There is a $\pi_{1}$-injective pared embedding $\iota: \widehat{N}_{1,1} \rightarrow \widehat{N}$, such that the representation $\sigma_{w_{1}, \ldots, w_{d}} \circ \iota_{*}: \pi_{1}\left(\hat{N}_{1,1}\right) \rightarrow \operatorname{PSL}(2, \mathbb{C})$ is conjugate to the extension of $\left.\sigma\right|_{\pi_{1}\left(N_{1,1}\right)}$ by $w_{1}$. So this extended representation is discrete, faithful, geometrically finite, and minimally parabolic with respect to $\widehat{P}_{1,1}$. Note that we are again using that finitely generated subgroups of geometrically finite groups are geometrically finite, provided the domain of discontinuity is nonempty. Thus the extension of $\left.\sigma\right|_{\pi_{1}\left(N_{1,1}\right)}$ by $w_{1}$ lies in $\operatorname{MP}\left(\hat{N}_{1,1}, \hat{P}_{1,1}\right)$, and so by the definition of $\mathcal{A}_{1,1}$, we have $\Pi\left(\sigma, w_{1}, \ldots, w_{d}\right)=\left(\left.\sigma\right|_{\pi_{1}\left(N_{1,1}\right)}, w_{1}\right) \in \mathcal{A}_{1,1}$. If $\left(w_{1}, \ldots, w_{d}\right)=(\infty, \ldots, \infty)$ then it follows immediately from Lemma 5.4 and the definition of $\Pi$ that $\Pi(\sigma, \infty, \ldots, \infty)=$ $\left(\left.\sigma\right|_{\pi_{1}\left(N_{1,1}\right)}, \infty\right) \in \mathcal{A}_{1,1}$.

We now use Klein-Maskit combination to show that $\mathcal{A}_{1,1} \subset \Pi(\mathcal{A})$. We begin by defining some new pared manifolds that arise as pieces of $N$ and $\hat{N}$ (see Figure 4). The annulus $\gamma_{2} \times[0,1]$ divides $N$ into two pieces. Let $N_{1,1}$ denote the closure of the piece containing $\gamma_{1}$ and let $N_{3}$ denote the closure of the remaining piece containing $\gamma_{3}, \ldots, \gamma_{d}$. Let $\hat{N}_{1,1}=N_{1,1}-U_{1}$ and $\hat{N}_{3}=N_{3}-\bigcup_{i=3}^{d} U_{i}$. Define $\widehat{P}_{1,1}=\partial U_{1} \cup\left(\gamma_{2} \times[0,1]\right)$ and $\widehat{P}_{3}=\left(\bigcup_{i=3}^{d} \partial U_{i}\right) \cup\left(\gamma_{2} \times[0,1]\right)$.

Next define $N_{2}=N-\bigcup_{i \neq 2} U_{i}$ and set $P_{2}$ to be the union of the $d-1$ tori $\bigcup_{i \neq 2} \partial U_{i}$ with the annulus in $P \subset S \times\{1\}$ whose core curve is homotopic to $\gamma_{2}$. We then obtain $(\widehat{N}, \widehat{P})$ from $\left(N_{2}, P_{2}\right)$ by drilling out $\gamma_{2}$. Again, refer to Figure 4 .

For any $(\rho, w) \in \mathcal{A}_{1,1}, w \neq \infty$, we have $\rho_{w} \in M P\left(\hat{N}_{1,1}, \widehat{P}_{1,1}\right)$. We can choose a representation $\hat{\rho}_{1}: \pi_{1}\left(\hat{N}_{1,1}\right) \rightarrow \operatorname{PSL}(2, \mathbb{C})$ in the conjugacy class $\rho_{w}$ such that

$$
\hat{\rho}_{1}\left(\gamma_{2}\right)=\left(\begin{array}{ll}
1 & 2 \\
0 & 1
\end{array}\right) \text {. }
$$

Since $\rho_{w}$ is an extension of $\rho$, some representation $\sigma_{1}: \pi_{1}\left(N_{1,1}\right) \rightarrow \hat{\rho}_{1}\left(\pi_{1}\left(\hat{N}_{1,1}\right)\right)$ represents the conjugacy class $\rho \in M P_{0}\left(N_{1,1}, P_{1,1}^{\prime}\right)$. Note that the top and bottom of $\mathbb{H}^{3} / \sigma_{1}\left(\pi_{1}\left(N_{1,1}\right)\right)$ are well-defined: the conformal boundary component on the top of $\mathbb{H}^{3} / \sigma_{1}\left(\pi_{1}\left(N_{1,1}\right)\right)$ is a thrice-punctured sphere, and the bottom is a punctured torus. For any $\hat{\rho}_{1}$ chosen as above, there is some $C_{1}$ such that $B^{+}=\left\{z \in \mathbb{C}: \operatorname{Im}(z)>C_{1}\right\}$ and $B^{-}=\left\{z \in \mathbb{C}: \operatorname{Im}(z)<-C_{1}\right\}$ are precisely invariant for the subgroup $\left\langle\hat{\rho}_{1}\left(\gamma_{2}\right)\right\rangle$ 


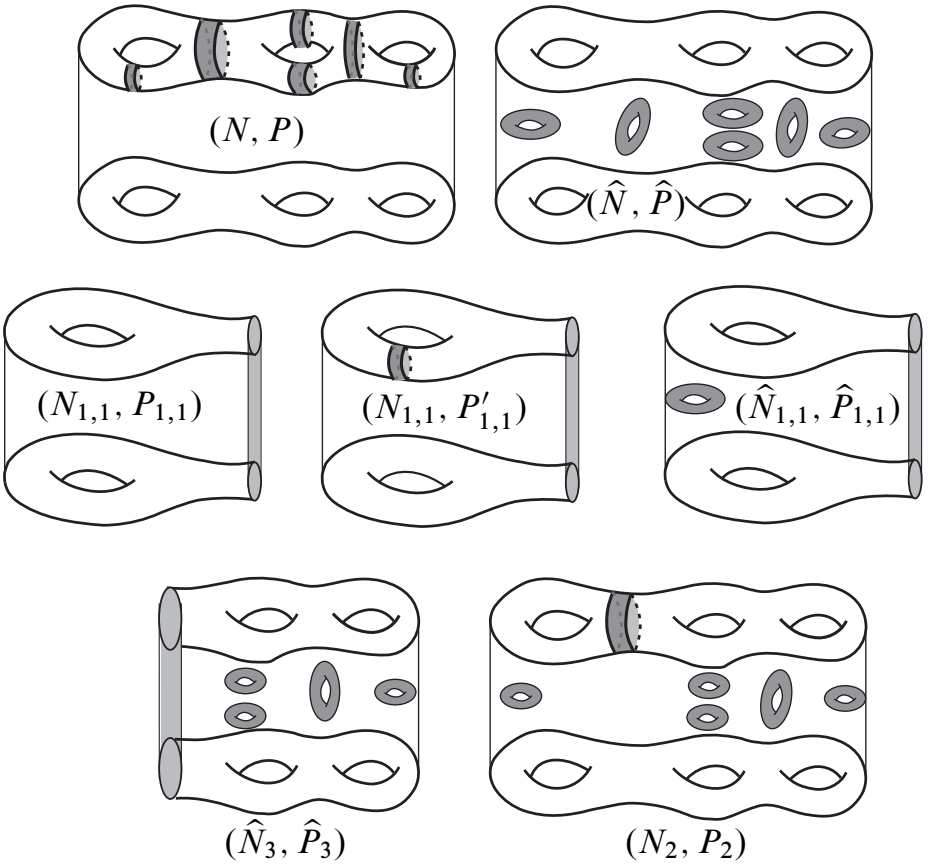

Figure 4: Some of the pared manifolds we are using (illustrated in genus 3 ). The shaded regions indicate the paring locus.

in $\hat{\rho}_{1}\left(\pi_{1}\left(\widehat{N}_{1,1}\right)\right)$. Without loss of generality, we can assume that $\hat{\rho}_{1}$ has been chosen such that $B^{+} \subset \Omega\left(\hat{\rho}_{1}\right) \subset \Omega\left(\sigma_{1}\right)$ projects to the top surface of $\Omega\left(\sigma_{1}\right) / \sigma_{1}\left(\pi_{1}\left(\hat{N}_{1,1}\right)\right)$. Next, let $\widehat{M}_{3}$ be a geometrically finite hyperbolic 3-manifold homeomorphic to the interior of $\widehat{N}_{3}$ whose only cusps are those associated to $\hat{P}_{3}$. Let $h_{3}$ be an orientation preserving pared homeomorphism from $\left(\hat{N}_{3}, \hat{P}_{3}\right)$ to the relative compact core of $\widehat{M}_{3}$. The boundary $\partial \widehat{N}_{3}-\widehat{P}_{3}$ has a top and bottom component, both of which are homeomorphic to a punctured genus $g-1$ surface $S_{g-1,1}$. We will call these $S_{g-1,1, \text { top }}$ and $S_{g-1,1, \text { bot }}$. The homeomorphism $h_{3}$ distinguishes a top and bottom component of the relative compact core of $\widehat{M}_{3}$ and thus distinguishes a top and bottom of the conformal boundary of $\widehat{M}_{3}$. Define a representation $\widehat{\rho}_{3}: \pi_{1}\left(\widehat{N}_{3}\right) \rightarrow \operatorname{PSL}(2, \mathbb{C})$ conjugate to $\left(h_{3}\right)_{*}$ such that

(a) $\hat{\rho}_{3}\left(\gamma_{2}\right)=\left(\begin{array}{ll}1 & 2 \\ 0 & 1\end{array}\right)$,

(b) $B_{3}=\left\{z \in \mathbb{C}: \operatorname{Im}(z)>-C_{1}-1\right\}$ is a precisely invariant set for the subgroup $\left\langle\hat{\rho}_{3}\left(\gamma_{2}\right)\right\rangle$ in $\hat{\rho}_{3}\left(\pi_{1}\left(\widehat{N}_{3}\right)\right)$,

(c) the component of $\Omega\left(\hat{\rho}_{3}\right)$ containing $B_{3}$ projects to the bottom conformal boundary component of $\widehat{M}_{3}$. 
Let $\sigma_{3}=\left.\hat{\rho}_{3}\right|_{\pi_{1}\left(S_{g-1,1, \text { bot }}\right)}$. That is, $\sigma_{3}$ is the restriction of $\hat{\rho}_{3}$ to the natural inclusion of the fundamental group of the bottom surface into $\pi_{1}\left(\hat{N}_{3}\right)$. Thus, $\mathbb{H}^{3} / \sigma_{3}\left(\pi_{1}\left(S_{g-1,1}\right)\right)$ has a rank-1 cusp associated to each of the curves $\gamma_{2}, \ldots, \gamma_{d}$. The cusp corresponding to $\gamma_{2}$ corresponds to the boundary of $S_{g-1,1}$, and the cusps corresponding to $\gamma_{3}, \ldots, \gamma_{d}$ are on the top since the representation $\sigma_{3}$ was constructed from the inclusion of the bottom surface into $\widehat{M}_{3}$. Hence, $\sigma_{3} \in M P_{0}\left(S_{g-1,1} \times I,\left(S_{g-1,1} \times\{1\}\right) \cap P\right)$.

Now we can apply type I Klein-Maskit combination along the subgroup $\left\langle\hat{\rho}_{3}\left(\gamma_{2}\right)\right\rangle=$ $\left\langle\hat{\rho}_{1}\left(\gamma_{2}\right)\right\rangle$. See Section 2.1 for references and notation. Observe that $\pi_{1}\left(N_{2}\right) \cong$ $\pi_{1}\left(\hat{N}_{1,1}\right) *\left\langle\gamma_{2}\right\rangle \pi_{1}\left(\hat{N}_{3}\right)$. Define a representation $\rho_{2}: \pi_{1}\left(N_{2}\right) \rightarrow \operatorname{PSL}(2, \mathbb{C})$ by setting $\rho_{2}(x)=\hat{\rho}_{3}(x)$ for all $x \in \pi_{1}\left(\hat{N}_{3}\right)$ and $\rho_{2}(x)=\hat{\rho}_{1}(x)$ for all $x \in \pi_{1}\left(\widehat{N}_{1,1}\right)$. By construction, the representation $\rho_{2}$ is discrete, faithful, geometrically finite, and minimally parabolic with respect to the paring locus $P_{2}$.

We can also apply Klein-Maskit combination to the subgroups $\sigma_{1}\left(\pi_{1}\left(S_{1,1}\right)\right)$ and $\sigma_{3}\left(\pi_{1}\left(S_{g-1,1}\right)\right)$. Since $\pi_{1}(S) \cong \pi_{1}\left(S_{1,1}\right) *\left\langle\gamma_{2}\right\rangle \pi_{1}\left(S_{g-1,1}\right)$, we can define a representation $\sigma: \pi_{1}(S) \rightarrow \operatorname{PSL}(2, \mathbb{C})$ by $\sigma(x)=\sigma_{1}(x)$ for all $x \in \pi_{1}\left(S_{1,1}\right)$ and $\sigma(x)=\sigma_{3}(x)$ for $x \in \pi_{1}\left(S_{g-1,1}\right)$. This defines a discrete, faithful, geometrically finite representation whose parabolics consist precisely of the curves $\gamma_{i}$ in $S$. Since $\sigma_{1} \in M P_{0}\left(N_{1,1}, P_{1,1}^{\prime}\right)$ and $\sigma_{3} \in M P_{0}\left(S_{g-1,1} \times I,\left(S_{g-1,1} \times\{1\}\right) \cap P\right)$, the cusps corresponding to $\gamma_{1}, \gamma_{3}, \ldots, \gamma_{d}$ are in the top of $\mathbb{H}^{3} / \sigma\left(\pi_{1}(S)\right)$. Moreover, since $B^{-}$ and $B_{3}$ cover the bottoms of their respective manifolds, the cusp associated to $\gamma_{2}$ is also in the top. It follows that $\sigma \in M P_{0}(N, P)$.

To summarize, we now have a representation $\sigma \in M P_{0}(N, P)$ such that $\left.\sigma\right|_{\pi_{1}\left(N_{1,1}\right)}=\sigma_{1}$ is conjugate to $\rho$. The next step is to find $w_{1}, \ldots, w_{d}$ with $\Pi\left(\sigma, w_{1}, \ldots, w_{d}\right)=(\rho, w)$. Since $\pi_{1}(\hat{N})$ is generated by $\pi_{1}\left(N_{2}\right)$ and the meridian of the second torus boundary component $\partial U_{2}$, we can extend the representation $\rho_{2}$ to a representation $\hat{\rho}: \pi_{1}(\hat{N}) \rightarrow$ $\operatorname{PSL}(2, \mathbb{C})$ by defining $\hat{\rho}$ to equal $\rho_{2}$ on $\pi_{1}\left(N_{2}\right)$ and sending the additional generator to

$$
\left(\begin{array}{cc}
1 & w_{2} \\
0 & 1
\end{array}\right)
$$

for $w_{2} \in \mathbb{C}$. There is some constant $C_{2}$ such that if $\operatorname{Im}\left(w_{2}\right)>C_{2}$, then we can apply type II Klein-Maskit combination (see Section 2.1). In this case, $\hat{\rho}$ defines a geometrically finite representation of $\pi_{1}(\hat{N})$ which is minimally parabolic with respect to $(\hat{N}, \widehat{P})$.

On each torus boundary component of $\hat{N}$ there is a well-defined meridian. Up to conjugation (that depends on $i$ ), we can assume $\hat{\rho}$ sends $\gamma_{i}$ to

$$
\left(\begin{array}{ll}
1 & 2 \\
0 & 1
\end{array}\right)
$$


In this case, there will be some $w_{i}$ such that the meridian of $\partial U_{i}$ is sent to

$$
\left(\begin{array}{cc}
1 & w_{i} \\
0 & 1
\end{array}\right)
$$

With this definition of $w_{i}$ we now have a point $\left(\sigma, w_{1}, \ldots, w_{d}\right) \in \mathcal{A}$.

Next, we check that $w_{1}=w$ and therefore $\Pi\left(\sigma, w_{1}, \ldots, w_{d}\right)=(\rho, w)$. This follows by construction. The extension of $\sigma$ by $\left(w_{1}, \ldots, w_{d}\right)$ is conjugate to $\hat{\rho}$. The restriction of $\hat{\rho}$ to $\pi_{1}\left(\hat{N}_{1,1}\right)$ is conjugate to $\rho_{w}$ (which is conjugate to $\left.\hat{\rho}_{1}\right)$. Thus $w_{1}=w$.

Finally, if $(\rho, \infty) \in \mathcal{A}_{1,1}$, then we can pick any $w^{\prime} \neq \infty$ such that $\left(\rho, w^{\prime}\right) \in \mathcal{A}_{1,1}$. Following the same construction, we can find a point $\left(\sigma, w^{\prime}, w_{2}, \ldots, w_{d}\right) \in \mathcal{A}$ such that $\left.\sigma\right|_{\pi_{1}\left(N_{1,1}\right)}$ is conjugate to $\rho$. Then the point $(\sigma, \infty, \ldots, \infty)$ is also in $\mathcal{A}$ and satisfies $\Pi(\sigma, \infty, \ldots, \infty)=(\rho, \infty)$.

Recall that each $\gamma_{i}$ was contained in a four-punctured sphere or punctured torus component of $S-\bigcup_{j \neq i} \gamma_{j}$. Given $\sigma \in M P_{0}(N, P)$, one can define $\Pi_{i}$ similarly to $\Pi=\Pi_{1}$. The first coordinate is obtained by restricting $\sigma$ to the $i$-th such subsurface and the second coordinate is defined by projecting $\left(w_{1}, \ldots, w_{d}\right) \mapsto w_{i}$. Lemma 5.5 generalizes to show that $\Pi_{i}(\mathcal{A})=\mathcal{A}_{1,1}$ if $\gamma_{i}$ lives in a punctured torus or $\Pi_{i}(\mathcal{A})=$ $\mathcal{A}_{0,4}$ if $\gamma_{i}$ lives in a four-punctured sphere. In fact, for $i>1$, we will only need the first paragraph of the proof of this Lemma which shows that $\Pi_{i}(\mathcal{A}) \subset \mathcal{A}_{1,1}$ or $\Pi_{i}(\mathcal{A}) \subset \mathcal{A}_{0,4}$.

We now get the following corollary to Lemma 5.3 and Lemma 5.5.

Corollary 5.6 For all $\left(\sigma, w_{1}, \ldots, w_{d}\right) \in \mathcal{A}$ with $\left(w_{1}, \ldots, w_{d}\right) \neq(\infty, \ldots, \infty)$, the imaginary part of $w_{i}$ is bounded below by

$$
\operatorname{Im}\left(w_{i}\right)>1
$$

for all $i$.

\section{$5.4 \mathcal{A}$ is not locally connected}

In [20, Lemma 4.14], Bromberg shows that there exists a point $\left(\sigma_{z}, \infty\right) \in \mathcal{A}_{1,1}$ at which $\mathcal{A}_{1,1}$ fails to be locally connected. We will use Lemma 5.5 to extend this failure of local connectivity to $\mathcal{A}$. Although our statement of Lemma 5.7 differs from Bromberg's statement in [20], his proof implies this result (see also [47, Lemma 5.6] for a detailed proof). 
Lemma 5.7 (Bromberg [20]) There exists a point $z_{0} \in \mathcal{M}^{+}$, a closed rectangle $R$, and constants $\delta, c>0$ such that if $z$ lies in the $\delta$-neighborhood, $O$, of $z_{0}$ in $\mathbb{C}$ then $z \in \mathcal{M}^{+}$and the set

$$
\mathcal{A}_{z}=\left\{w \in \widehat{\mathbb{C}}:\left(\sigma_{z}, w\right) \in \mathcal{A}_{1,1}\right\}
$$

satisfies

(i) $\mathcal{A}_{z} \cap \operatorname{int}(R) \neq \varnothing$,

(ii) The distance between $\overline{\mathcal{A}_{z}}$ and $\partial R$ is at least $c$.

Moreover, we can choose $R$ such that its sides are parallel to the axes and its width is $<2$.

Let $W$ be an open neighborhood of $\sigma_{z_{0}}$ in $M P_{0}\left(N_{1,1}, P_{1,1}^{\prime}\right)$ such that for all $\sigma_{z} \in \bar{W}$, $z \in O$. In other words, if $\tau: \mathcal{M}^{+} \rightarrow M P_{0}\left(N_{1,1}, P_{1,1}^{\prime}\right)$ is the homeomorphism $z \mapsto \sigma_{z}$, then $W$ is a neighborhood of $\sigma_{z_{0}}$ such that $\tau^{-1}\left(\frac{W}{W}\right) \subset O$.

By Lemma 5.7, $\mathcal{A}_{z_{0}} \cap \operatorname{int}(R) \neq \varnothing$, so let $u_{1} \in \operatorname{int}(R)$ such that $\left(\sigma_{z_{0}}, u_{1}\right) \in \mathcal{A}_{1,1}$. Lemma 5.5 shows that $\Pi: \mathcal{A} \rightarrow \mathcal{A}_{1,1}$ is a surjection, so there is some $\left(\sigma_{0}, u_{1}, \ldots, u_{d}\right) \in$ $\Pi^{-1}\left(\sigma_{z_{0}}, u_{1}\right)$. Note that since $\left(\sigma_{0}, u_{1}, \ldots, u_{d}\right) \in \mathcal{A}$, we have $\sigma_{0} \in M P_{0}(N, P)$. Thus, by the definition of $\mathcal{A}$, we also have $\left(\sigma_{0}, \infty, \ldots, \infty\right) \in \mathcal{A}$.

We now claim that $\left(\sigma_{0}, u_{1}+2 n, \ldots, u_{d}+2 n\right) \in \mathcal{A}$ for all $n$. The definition of $\left(\sigma_{0}, u_{1}, \ldots, u_{d}\right)$ belonging to the set $\mathcal{A}$ is that the extension of $\sigma_{0}$ by $\left(u_{1}, \ldots, u_{d}\right)$ to a representation of $\widehat{N}$ lies in $M P(\widehat{N}, \widehat{P})$. The extension of $\sigma_{0}$ by $\left(u_{1}+2 n, \ldots, u_{d}+2 n\right)$ (as in Section 5.2) has the same image in $\operatorname{PSL}(2, \mathbb{C})$ as the extension of $\sigma_{0}$ by $\left(u_{1}, \ldots, u_{d}\right)$ since the two representations differ by precomposition by an automorphism of $\pi_{1}(\hat{N})$. The automorphism is the one induced by Dehn twists in the collection of annuli $\left\{\left(\gamma_{i} \times\left[\frac{1}{2}, 1\right]\right) \cap \widehat{N}\right\}$ in $\hat{N}$ whose core curves are homotopic to the collection of $\gamma_{i}$ curves, and run from the torus boundary components to one of nontorus boundary components. Hence, $\left(\sigma_{0}, u_{1}+2 n, \ldots, u_{d}+2 n\right) \in \mathcal{A}$ for all $n$.

Let $U$ be a neighborhood of $\left(\sigma_{0}, \infty, \ldots, \infty\right)$ in $\mathcal{A}$ such that for all $\left(\sigma, w_{1}, \ldots, w_{d}\right)$ in $U$, the first coordinate of $\Pi\left(\sigma, w_{1}, \ldots, w_{d}\right)$ lies in $W$. In other words, for all $\left(\sigma, w_{1}, \ldots, w_{d}\right) \in U,\left.\sigma\right|_{\pi_{1}\left(N_{1,1}\right)} \in W$.

For each $n$, let $C_{n}$ be the collection of components of $U$ defined by

$$
\left.C_{n}=U \cap \Pi^{-1}(W \times(R+2 n))\right) .
$$

Equivalently,

$$
C_{n}=\left\{\left(\sigma, w_{1}, \ldots, w_{d}\right) \in U: w_{1} \in(R+2 n)\right\} .
$$

Here, $R+2 n$ denotes the box $R$ translated by $2 n$. 
We have already produced a sequence of points

$$
\left(\sigma_{0}, u_{1}+2 n, \ldots, u_{d}+2 n\right) \in \Pi^{-1}(W \times(R+2 n))
$$

converging to $\left(\sigma_{0}, \infty, \ldots, \infty\right)$; hence, $C_{n}$ is nonempty for all but finitely many $n$. Now we claim that $\overline{C_{n}}$ and $\overline{U-C_{n}}$ are uniformly bounded apart from each other.

Lemma 5.8 There exists some $c>0$ such that for all $n$, if $\left(\sigma, w_{1}, \ldots, w_{d}\right) \in \overline{C_{n}}$ and $\left(\sigma^{\prime}, w_{1}^{\prime}, \ldots, w_{d}^{\prime}\right) \in \overline{U-C_{n}}$ then

$$
\left|w_{1}-w_{1}^{\prime}\right|>c
$$

Proof For any $\left(\sigma, w_{1}, \ldots, w_{d}\right) \in \bar{U}$, we have $\left.\sigma\right|_{\pi_{1}\left(N_{1,1}\right)} \in \bar{W}$. Since $\tau^{-1}(\bar{W}) \subset O$, if we let $z=\tau\left(\left.\sigma\right|_{\pi_{1}\left(N_{1,1}\right)}\right)$, then Lemma 5.7 implies that $\overline{\mathcal{A}_{z}}$ and $\partial R$ are at least a distance $c$ apart. It is a direct consequence of the symmetry in the Maskit slice (Lemma 5.1) and Lemma 5.2 that the set $\mathcal{A}_{z}$ is invariant under the translation $w \mapsto w+2$. Thus, points in $\overline{\mathcal{A}_{z}} \cap(R+2 n)$ are bounded away from points in $\overline{\mathcal{A}_{z}} \cap(\mathbb{C}-(R+2 n))$ by a distance of at least $2 c>c$.

Since $C_{n}$ is nonempty for all but finitely many $n$ and the sets $C_{n}$ accumulate to $\left(\sigma_{0}, \infty, \ldots, \infty\right)$, Lemma 5.8 implies that any neighborhood $U^{\prime} \subset U$ containing $\left(\sigma_{0}, \infty, \ldots, \infty\right), U^{\prime}$ has infinitely many components. Thus we have shown:

Proposition 5.9 There is a point $\sigma_{0} \in M P_{0}(N, P)$ such that $\mathcal{A}$ is not locally connected at $\left(\sigma_{0}, \infty, \ldots, \infty\right)$.

Remark We do not need the closures of $C_{n}$ and its complement to be disjoint to conclude that $\mathcal{A}$ is not locally connected, but we will need the full strength of Lemma 5.8 in the following section.

\subsection{Definition of $\Phi$}

Now that we have defined $\mathcal{A}$ and shown that it fails to be locally connected at some point $\left(\sigma_{0}, \infty, \ldots, \infty\right)$, we want to construct a map $\Phi$ from a subset of $\mathcal{A}$ containing this point into $A H(S \times I)$. In this section, we show that $\Phi$ is well-defined on some subset of $\mathcal{A}$ and in the subsequent sections, we will show that $\Phi$ is a local homeomorphism from $\mathcal{A}$ to $M P(N) \cup M P_{0}(N, P) \subset A H(N)$ at the point $\left(\sigma_{0}, \infty, \ldots, \infty\right)$.

As in [20, Section 3], we construct the map $\Phi$ in two steps. Heuristically, the points in $\mathcal{A}$ satisfying $\left(w_{1}, \ldots, w_{d}\right)=(\infty, \ldots, \infty)$ parameterize $M P_{0}(N, P)$, while the points $\left(\sigma, w_{1}, \ldots, w_{d}\right) \in \mathcal{A}$ parameterize a subset of $M P(\hat{N}, \widehat{P})$. For points 
$(\sigma, \infty, \ldots, \infty) \in \mathcal{A}$, the representation $\sigma \in M P_{0}(N, P) \subset A H(N)$, so we will define $\Phi(\sigma, \infty, \ldots, \infty)=\sigma$. For all other points $\left(\sigma, w_{1}, \ldots, w_{d}\right) \in \mathcal{A}$, the representation $\sigma_{\left(w_{1}, \ldots, w_{d}\right)} \in M P(\hat{N}, \hat{P})$ and so $\mathbb{H}^{3} / \sigma_{\left(w_{1}, \ldots, w_{d}\right)}\left(\pi_{1}(\hat{N})\right)$ is a marked hyperbolic manifold with $d$ rank-2 cusps. For these points, we will define $\Phi\left(\sigma, w_{1}, \ldots, w_{d}\right)$ to be the marked hyperbolic manifold in $M P(N)$ obtained by filling in these cusps. We use the filling theorem to show that $\Phi$ is well-defined on some subset of $\mathcal{A}$ and that $\Phi$ is continuous.

Let $(\sigma, w) \in \mathcal{A}$ such that $w \neq(\infty, \ldots, \infty)$. By the definition of $\mathcal{A}$ we have that $\sigma_{w} \in M P(\widehat{N}, \widehat{P})$. Let $\widehat{M}_{\sigma, w}=\mathbb{H}^{3} / \sigma_{w}\left(\pi_{1}(\hat{N})\right)$ be the corresponding geometrically finite manifold with $d$ cusps.

Recall that $\epsilon_{3}$ denotes the Margulis constant in dimension 3. By Corollary 4.13, there is a constant $K$ such that if

$$
\frac{\left|w_{i}\right|}{\sqrt{2 \operatorname{Im}\left(w_{i}\right)}}>K
$$

for all $i$, then we can $\beta_{i}$-fill the $i$-th cusp $(i=1, \ldots, d)$ to get a hyperbolic manifold $M_{\sigma, w}$ with the same conformal boundary as $\widehat{M}_{\sigma, w}$, and there exists a bilipschitz diffeomorphism

$$
\phi_{\sigma, w}: \widehat{M}_{\sigma, w}-\mathbb{T}_{\epsilon_{3}}(T) \rightarrow M_{\sigma, w}-\mathbb{T}_{\epsilon_{3}}(\gamma) .
$$

Here $T$ denotes the union of the cusps $T_{i}$ and $\gamma$ denotes the union of the curves $\gamma_{i}$.

Define

$$
\mathcal{A}_{K}=\left\{(\sigma, w) \in \mathcal{A}: w=(\infty, \ldots, \infty) \text { or } \frac{\left|w_{i}\right|}{\sqrt{2 \operatorname{Im}\left(w_{i}\right)}}>K \text { for all } i\right\} .
$$

Recall that $\sigma \in M P_{0}(N, P)$ can be identified with a marked hyperbolic manifold $\left(M_{\sigma}, f_{\sigma}\right)$. Without loss of generality, we can assume that $f_{\sigma}$ is a smooth immersion and that $f_{\sigma}(N)$ does not intersect the $\epsilon_{3}$-parabolic thin part of $M_{\sigma}$ since $f_{\sigma}$ is only defined up to homotopy. As $\sigma\left(\pi_{1}(N)\right)$ is a subgroup of $\sigma_{w}\left(\pi_{1}(\hat{N})\right)$ we have a covering map

$$
\pi_{\sigma, w}: M_{\sigma} \rightarrow \widehat{M}_{\sigma, w}
$$

Now define

$$
f_{\sigma, w}=\phi_{\sigma, w} \circ \pi_{\sigma, w} \circ f_{\sigma} .
$$

Since we have assumed $f_{\sigma}(N)$ avoids the $\epsilon_{3}$-parabolic thin part of $M_{\sigma}, \pi_{\sigma, w} \circ f_{\sigma}(N)$ is contained in $\widehat{M}_{\sigma, w}-\mathbb{T}_{\epsilon_{3}}(T)$. Thus, postcomposition by the filling map $\phi_{\sigma, w}$ makes sense here.

We next claim (as in [20, Lemma 3.6]) that $\left(f_{\sigma, w}\right)_{*}$ is an isomorphism from $\pi_{1}(N)$ to $\pi_{1}\left(M_{\sigma, w}\right)$ and therefore $\left(M_{\sigma, w}, f_{\sigma, w}\right) \in A H(N)$. First observe that $f_{\sigma}$ is a homotopy 
equivalence so we only need to show that

$$
\left(\phi_{\sigma, w}\right)_{*} \circ\left(\pi_{\sigma, w}\right)_{*}: \pi_{1}\left(M_{\sigma}\right) \rightarrow \pi_{1}\left(M_{\sigma, w}\right)
$$

is an isomorphism. Recall $\pi_{1}(\hat{N})=\left\langle\pi_{1}(N), \beta_{1}, \ldots, \beta_{d} \mid\left[\beta_{i}, \gamma_{i}\right]=1\right\rangle$. By the definition of the covering map $\pi_{\sigma, w}$ and the definition of the representation $\sigma_{w}$,

$$
\pi_{1}\left(\widehat{M}_{\sigma, w}\right)=\left\langle\left(\pi_{\sigma, w}\right)_{*}\left(\pi_{1}\left(M_{\sigma}\right)\right), \sigma_{w}\left(\beta_{1}\right), \ldots, \sigma_{w}\left(\beta_{d}\right) \mid\left[\sigma_{w}\left(\beta_{i}\right), \sigma_{w}\left(\gamma_{i}\right)\right]=1\right\rangle .
$$

Now the filling map $\left(\phi_{\sigma, w}\right)_{*}$ kills the meridians in $\widehat{M}_{\sigma, w}$ which were precisely the group elements $\sigma_{w}\left(\beta_{i}\right)$. Thus

$$
\left(\pi_{\sigma, w}\right)_{*}\left(\pi_{1}\left(M_{\sigma}\right)\right) \cap \operatorname{Ker}\left(\left(\phi_{\sigma, w}\right)_{*}\right)=\{1\}
$$

and therefore $\left(\phi_{\sigma, w}\right)_{*} \circ\left(\pi_{\sigma, w}\right)_{*}$ is an isomorphism from $\pi_{1}\left(M_{\sigma}\right)$ onto its image, which is $\pi_{1}\left(M_{\sigma, w}\right)$.

Moreover, as the filling preserves the conformal boundary components of $\widehat{M}_{\sigma, w}$ and the filled manifold $M_{\sigma, w}$ has no cusps, $\left(f_{\sigma, w}\right)_{*}$ is a minimally parabolic, geometrically finite representation in $A H(N)$.

So we define

$$
\Phi(\sigma, w)= \begin{cases}\left(f_{\sigma, w}\right)_{*} & \text { if } w \neq(\infty, \ldots, \infty) \\ \sigma & \text { if } w=(\infty, \ldots, \infty)\end{cases}
$$

Therefore we have defined $\Phi$ on some subset $\mathcal{A}_{K} \subset \mathcal{A}$ that satisfies $\Phi\left(\mathcal{A}_{K}\right) \subset$ $M P(N) \cup M P_{0}(N, P)$.

Lemma 5.10 The map $\Phi$ is continuous on $\mathcal{A}_{K}$.

Proof Let $\left(\sigma_{0}, w_{0}\right)$ be a point in $\mathcal{A}_{K}$ with $w_{0} \neq(\infty, \ldots, \infty)$. Let $B$ be the component of $\left(\mathcal{A}_{K}-\{(\sigma, w): w=(\infty, \ldots, \infty)\}\right)$ containing $\left(\sigma_{0}, w_{0}\right)$. Clearly the correspondence $(\sigma, w) \mapsto \sigma_{w}$ is a continuous map from $\left(\mathcal{A}_{K}-\{(\sigma, w): w=(\infty, \ldots, \infty)\}\right)$ to $M P(\widehat{N}, \widehat{P})$, so takes the component $B$ into one of the components $C$ of $M P(\widehat{N}, \widehat{P})$. Recall from Section 2 that $C=F^{-1}\left(\left[\left(\hat{N}_{C}, \hat{P}_{C}\right), h_{C}\right]\right)$ for some $\left[\left(\hat{N}_{C}, \hat{P}_{C}\right), h_{C}\right] \in$ $A(\widehat{N}, \widehat{P})$. For any point $\left(\widehat{M}_{\hat{\rho}}, f_{\hat{\rho}}\right) \in C$, the map $f_{\widehat{\rho}} \circ h_{C}^{-1}$ is homotopic to a pared homeomorphism from $\left(\widehat{N}_{C}, \widehat{P}_{C}\right)$ to the relative compact core of $\widehat{M}_{\hat{\rho}}$, and thus we can use $f_{\hat{\rho}} \circ h_{C}^{-1}$ to define a marking from $\partial \hat{N}_{C}-\widehat{P}_{C}$ to the conformal boundary of $\widehat{M}_{\hat{\rho}}$. The Ahlfors-Bers parameterization $\widehat{\mathcal{A B}}_{C}: C \rightarrow \mathcal{T}\left(\partial \widehat{N}_{C}-\widehat{P}_{C}\right)$ is defined by sending $\left(\widehat{M}_{\widehat{\rho}}, f_{\widehat{\rho}}\right)$ to the conformal boundary of $\widehat{M}_{\widehat{\rho}}$ marked by $f_{\widehat{\rho}} \circ h_{C}^{-1}$. Similarly, let $\mathcal{A B}: M P(N) \rightarrow \mathcal{T}(\partial N)$ be the Ahlfors-Bers parameterization of $M P(N)$.

For any $(\sigma, w) \in B$, we showed in the definition of $\Phi$ that $\left(f_{\sigma, w}\right)_{*}$ is an isomorphism. Thus the $\bigcup h_{C}\left(\beta_{i}\right)$-Dehn filling of $\left(\hat{N}_{C}, \widehat{P}_{C}\right)$ has fundamental group isomorphic 
to $\pi_{1}(N)$, a surface group, and therefore is homeomorphic to $N$ [32]. Here, the $\bigcup h_{C}\left(\beta_{i}\right)$-Dehn filling refers to the collection of filling slopes corresponding to $\beta_{1}, \ldots, \beta_{d}$ under the homotopy equivalence $h_{C}:(\widehat{N}, \widehat{P}) \rightarrow\left(\widehat{N}_{C}, \widehat{P}_{C}\right)$. This filling gives us an inclusion $i_{C}:\left(\hat{N}_{C}, \widehat{P}_{C}\right) \rightarrow N$ which defines a homeomorphism $i_{C}:\left(\partial \widehat{N}_{C}-\widehat{P}_{C}\right) \rightarrow \partial N$. Using this homeomorphism, we can identify $\mathcal{T}\left(\partial \widehat{N}_{C}-\widehat{P}_{C}\right)$ with $\mathcal{T}(\partial N) \cong \mathcal{T}(S) \times \mathcal{T}(S)$.

With this identification of the Teichmüller spaces of $\left(\partial \widehat{N}_{C}-\widehat{P}_{C}\right)$ and $\partial N$, it follows that $\Phi(\sigma, w)=\mathcal{A B}^{-1} \circ \widehat{\mathcal{A B}}_{C}\left(\sigma_{w}\right)$ for any $(\sigma, w) \in B$ since the filling map $\phi_{\sigma, w}$ extends to a conformal map from the conformal boundary of $\widehat{M}_{\sigma, w}$ to the conformal boundary of $M_{\sigma, w}$. Since the Ahlfors-Bers maps are homeomorphisms, this shows that $\Phi$ is continuous on the component $B$ of $\left(\mathcal{A}_{K}-\{(\sigma, w): w=(\infty, \ldots, \infty)\}\right)$ containing $\left(\sigma_{0}, w_{0}\right)$. Since $\left(\sigma_{0}, w_{0}\right)$ was arbitrary, we have that $\Phi$ is continuous on all of $\left(\mathcal{A}_{K}-\{(\sigma, w): w=(\infty, \ldots, \infty)\}\right)$.

Next, we show $\Phi$ is continuous at points where $w=(\infty, \ldots, \infty)$. Suppose

$$
\left(\sigma_{i}, w_{1, i}, \ldots, w_{d, i}\right) \rightarrow(\sigma, \infty, \ldots, \infty) .
$$

We claim that $\Phi\left(\sigma_{i}, w_{1, i}, \ldots, w_{d, i}\right) \rightarrow \Phi(\sigma, \infty, \ldots, \infty)=\sigma$. If $\left(w_{1, i}, \ldots, w_{d, i}\right)=$ $(\infty, \ldots, \infty)$ for all $i$, then clearly $\Phi\left(\sigma_{i}, w_{1, i}, \ldots, w_{d, i}\right)=\sigma_{i} \rightarrow \sigma$.

Now suppose that $\left(w_{1, i}, \ldots, w_{d, i}\right) \neq(\infty, \ldots, \infty)$ for all $i$. Let $\left(M_{\sigma}, f_{\sigma}\right)$ be the marked hyperbolic 3-manifold corresponding to $\sigma$. Again, assume that $f_{\sigma}$ is smooth. Since $\sigma_{i} \rightarrow \sigma$, there is a sequence $L_{i} \rightarrow 1$ and smooth homotopy equivalences $g_{i}: M_{\sigma} \rightarrow M_{\sigma_{i}}$ such that $\left(g_{i} \circ f_{\sigma}\right)_{*}=\sigma_{i}$ and $g_{i}$ is an $L_{i}$-bilipschitz local diffeomorphism on a compact core of $M_{\sigma}$ (ie, the maps $g_{i}$ converge to a local isometry). If we let $f_{\sigma_{i}}=g_{i} \circ f_{\sigma}$, then the pullback metrics on $N$ via $f_{\sigma_{i}}$ converge to the pullback metrics on $N$ via $f_{\sigma}$. See [13, page 154] for this geometric definition of algebraic convergence (see also McMullen [58, page 43]).

Recall, by definition, $\Phi\left(\sigma_{i}, w_{i}\right)=\left(M_{\sigma, w_{i}}, f_{\sigma_{i}, w_{i}}\right)$, where $f_{\sigma_{i}, w_{i}}=\phi_{\sigma_{i}, w_{i}} \circ \pi_{\sigma_{i}, w_{i}} \circ f_{\sigma_{i}}$. (Notation: we abbreviate the $d$-tuple $\left(w_{1, i}, \ldots, w_{d, i}\right)$ by $w_{i}$ and use a double subscript to refer to the $j$-th entry, $w_{j, i}$, of $w_{i}$.)

Since for each $j, w_{j, i} \rightarrow \infty$ as $i \rightarrow \infty$, we can find a sequence $J_{i} \rightarrow 1$ such that $\phi_{\sigma_{i}, w_{i}}$ is $J_{i}$-bilipschitz away from the $\epsilon_{3}$-neighborhood of the cusps of $\widehat{M}_{\sigma_{i}, w_{i}}$. In particular, $\phi_{\sigma_{i}, w_{i}}$ is $J_{i}$-bilipschitz on $\pi_{\sigma_{i}, w_{i}}\left(f_{\sigma_{i}}(N)\right)$.

It follows that the limit of the pullback metrics on $N$ via the maps $f_{\sigma_{i}, w_{i}}: N \rightarrow M_{\sigma_{i}, w_{i}}$ is the same as the limit of the pullback metrics on $N$ via $\pi_{\sigma_{i}, w_{i}} \circ f_{\sigma_{i}}$ since $f_{\sigma_{i}, w_{i}}=$ $\phi_{\sigma_{i}, w_{i}} \circ \pi_{\sigma_{i}, w_{i}} \circ f_{\sigma_{i}}$. The covering map is a local isometry so this limit is the limit of the pullback metrics on $N$ under $f_{\sigma_{i}}$. Since $\sigma_{i} \rightarrow \sigma$, the limit of the pullback metrics 
on $N$ via $f_{\sigma_{i}}$ is the pullback metric on $N$ via $f_{\sigma}$. To summarize, the limit of the pullback metrics on $N$ via the maps $f_{\sigma_{i}, w_{i}}: N \rightarrow M_{\sigma_{i}, w_{i}}$ is the pullback metric on $N$ via $f_{\sigma}: N \rightarrow M_{\sigma}$. This convergence of metrics implies that $\left(f_{\sigma_{i}, w_{i}}\right)_{*}$ converges to $\sigma$ in $A H(N)$ [13].

Remark Lemma 5.10 is essentially the same as [20, Proposition 3.7], but when there are multiple cusps we need to use the multiple cusp version of the filling theorem (Corollary 4.13) which requires all of the $w$-coordinates to go to infinity. This is the why we have defined $\mathcal{A}$ to exclude points $\left(\sigma, w_{1}, \ldots, w_{d}\right)$ where some but not all of the $w$-coordinates are $\infty$.

\subsection{An inverse to $\Phi$}

We now construct a map $\Psi$ from a subset of $M P(N) \cup M P_{0}(N, P)$ to $\mathcal{A}$. For any $\sigma \in M P_{0}(N, P)$ and any sufficiently small neighborhood of $\sigma$ in $M P(N) \cup M P_{0}(N, P)$, $\Psi$ will be an inverse to $\Phi$.

Fix a representation $\sigma_{0} \in M P_{0}(N, P)$. For $\rho$ in some neighborhood of $\sigma_{0}$, the definition of $\Psi$ will have two coordinates $\Psi(\rho)=(\xi(\rho), q(\rho)) \in M P_{0}(N, P) \times \widehat{\mathbb{C}}^{d}$. We will actually begin by defining a neighborhood $V^{\prime}$ of $\sigma_{0}$ such that for $\rho \in V^{\prime}, \xi(\rho) \in$ $A H(N, P)$. We will then restrict to a smaller neighborhood $V$ such that $\xi(V) \subset$ $M P_{0}(N, P)$ and $(\xi(\rho), q(\rho)) \in \mathcal{A}$. Before defining this neighborhood of $\sigma_{0}$ on which $\Psi$ is defined, we set up some notation and background.

Let $\mathcal{H}(N)$ denote the space of smooth, hyperbolic metrics on $N$ with the $C^{\infty}$-topology (see $\left[26\right.$, I.1.1] for the definition of a $\left.\operatorname{PSL}(2, \mathbb{C}), \mathbb{H}^{3}\right)$-structure on a manifold with boundary, and I.1.5 for a description of the space $\mathcal{H}(N)$ which is denoted $\Omega(N)$ in [26]). If we let $\mathcal{D}(N)$ be the space of smooth developing maps $\tilde{N} \rightarrow \mathbb{H}^{3}$ with the compact $-C^{\infty}$ topology, then $\mathcal{H}(N)$ is the quotient of $\mathcal{D}(N)$ by $\operatorname{PSL}(2, \mathbb{C})$ acting by postcomposition. Note that $\mathcal{H}(N)$ is still infinite dimensional since we are not identifying developing maps that differ by the lift of an isotopy. Let $H: \mathcal{H}(N) \rightarrow X(N)$ be the holonomy map. Theorem I.1.7.1 of [26] locally describes $\mathcal{H}(N)$. See [26, Chapter I] for more details.

Theorem 5.11 (Canary-Epstein-Green [26]) Let $N_{\text {th }}$ be a thickening of $N$ (ie, the union of $N$ with a collar $\partial N \times I)$. Let $D_{0}: \tilde{N}_{\text {th }} \rightarrow \mathbb{H}^{3}$ be a fixed developing map. A small neighborhood of $\left.D_{0}\right|_{\tilde{N}}$ in $\mathcal{D}(N)$ is homeomorphic to $X \times Y$ where $X$ is a small neighborhood of the obvious inclusion $N \subset N_{\text {th }}$ in the space of locally flat embeddings, and $Y$ is a neighborhood of the holonomy map $H\left(D_{0}\right)$ in the representation variety $\mathcal{R}(N)$. A small neighborhood of $D_{0}$ in $\mathcal{H}(N)$ is homeomorphic to $X \times Z$ where $Z$ is a small neighborhood of the conjugacy class of $H\left(D_{0}\right)$ in the character variety $X(N)$. 
We now let $V^{\prime}$ be a neighborhood of $\sigma_{0} \in V^{\prime} \subset M P(N) \cup M P_{0}(N, P)$ that satisfies the properties (1)-(4) given below. Roughly, $V^{\prime}$ is a neighborhood on which we can define a section $\varsigma: V^{\prime} \subset A H(N) \rightarrow \mathcal{H}(N)$ and such that if $\rho \in V^{\prime}$ then the length of $\rho\left(\gamma_{i}\right)$ is short in $M_{\rho}$. The existence of such a neighborhood follows from the arguments given in [20, Section 3.1] and Theorem 5.11, although we include justification for why we can define $V^{\prime}$ with these properties after the statement of each property.

Fix a smooth embedding $s_{\sigma_{0}}: N \rightarrow M_{\sigma_{0}}$ such that $\left(s_{\sigma_{0}}\right)_{*}=\sigma_{0}$. Let $g_{\sigma_{0}}$ be the pullback of the hyperbolic metric on $N$. We can choose $s_{\sigma_{0}}$ so that the core curves of the annuli in $P$ (ie, the curves $\gamma_{i} \times\{1\}$ ) have length less than $\epsilon_{3} / 4$ in the $g_{\sigma_{0}}$ metric.

(1) There exists a continuous section $\varsigma: V^{\prime} \rightarrow \mathcal{H}(N)$ to the holonomy map such that $\varsigma\left(\sigma_{0}\right)=g_{\sigma_{0}}$.

The existence is given by Theorem 5.11. For any $\rho \in V^{\prime}$, define $g_{\rho}=\varsigma(\rho)$. We emphasize that, by the definition of a section, $H\left(g_{\rho}\right)=\rho$.

(2) For any $\rho_{1}, \rho_{2} \in V^{\prime}$, the identity map

$$
\left(N, g_{\rho_{1}}\right) \stackrel{\text { id }}{\rightarrow}\left(N, g_{\rho_{2}}\right)
$$

is 2-bilipschitz.

This follows from the continuity of $\varsigma$ and the topology on $\mathcal{H}(N)$.

(3) For any $\rho \in V^{\prime}$, there is a locally isometric immersion $s_{\rho}:\left(N, g_{\rho}\right) \rightarrow M_{\rho}$ where $M_{\rho}=\mathbb{H}^{3} / \rho\left(\pi_{1}(N)\right)$ is equipped with the hyperbolic metric, such that $\left(s_{\rho}\right)_{*}=\rho$. Moreover, there is some $\epsilon_{3}>\epsilon_{0}>0$ such that $s_{\rho}(N)$ is contained in the $\epsilon_{0}$-thick part of $M_{\rho}$.

The existence of $s_{\rho}: N \rightarrow M_{\rho}$ with $\left(s_{\rho}\right)_{*}=\rho$ is given by Theorem 5.11. We now find $\epsilon_{0}$. There is some $K$ such that for any point $x \in\left(N, g_{\sigma_{0}}\right)$, there are loops $\alpha, \beta$ based at $x$ of length less than $K$ such that the group generated by $\alpha$ and $\beta$ is not virtually abelian. For example, one can find a point $x_{0}$ and loops $\alpha_{0}$ and $\beta_{0}$ based at $x_{0}$ that generate a free group, and then let $K$ be larger than the sum of the diameter of $\left(N, g_{\sigma_{0}}\right)$ and the lengths of $\alpha_{0}$ and $\beta_{0}$. Since for any $\rho \in V^{\prime}$, id: $\left(N, g_{\sigma_{0}}\right) \rightarrow\left(N, g_{\rho}\right)$ is 2-bilipschitz by (2), at any point $x \in\left(N, g_{\rho}\right)$ there are loops based at $x$ of length less than $2 K$ generating a free group. There exists some $\epsilon_{3}>\epsilon_{0}>0$ such that for any component $\mathbb{T}_{\epsilon_{0}}$ of the $\epsilon_{0}$-thin part of any hyperbolic manifold $M$, the distance between $\partial \mathbb{T}_{\epsilon_{0}}$ and $\partial \mathbb{T}_{\epsilon_{3}}$ is at least $K$.

Suppose $s_{\rho}(x) \in s_{\rho}(N) \cap M_{\rho}^{\leq \epsilon_{0}}$ for some $x$. Then since $s_{\rho}$ is a homotopy equivalence, there are loops based at $s_{\rho}(x)$ that generate a free group and therefore must leave the $\epsilon_{3}$-thin part of $M_{\rho}$; however, to do so they must have length greater than $2 \mathrm{~K}$ 
contradicting that $s_{\rho}$ is a locally isometric immersion. Thus there exists some $\epsilon_{0}$ such that $s_{\rho}(N)$ is contained in $\epsilon_{0}$-thick part of $M_{\rho}$ for all $\rho \in V^{\prime}$.

(4) Let $\epsilon_{0}$ be the constant in property (3). Let $l_{0}$ be the constant from the drilling theorem such that the drilling map is a bilipschitz diffeomorphism outside an $\epsilon_{0}-$ Margulis tube about the drilling. Let $l_{1}=\min \left\{\epsilon_{0} / 8, l_{0}\right\}$. Then for any $\rho \in V^{\prime}$ we have the length of $\gamma_{i}$ in $M_{\rho}$ is less than $l_{1}$, for each $i=1, \ldots, d$. We can assume $V^{\prime}$ has this property since the length of any fixed curve $\gamma_{i}$ is a continuous function on the character variety.

Notation Here, the length of $\gamma_{i}$ in $M_{\rho}$ is really the length of the unique geodesic representative of $s_{\rho}\left(\gamma_{i}\right)$ in $M_{\rho}$. For the remainder of this section, we distinguish this geodesic representative by $s_{\rho}\left(\gamma_{i}\right)^{*}$. This curve is homotopic to $s_{\rho}\left(\gamma_{i} \times\{t\}\right)$ for any $t$, but its length is less than or equal to the length of $s_{\rho}\left(\gamma_{i} \times\{t\}\right)$. We make this distinction since we will also be using the length of $s_{\rho}\left(\gamma_{i} \times\{t\}\right)$, which is the length of $\gamma_{i} \times\{t\} \subset\left(N, g_{\rho}\right)$.

Now we construct the map $\xi$ which will be the first coordinate of $\Psi$. When $\rho \in$ $V^{\prime} \cap M P_{0}(N, P)$, set $\xi(\rho)=\rho$. Otherwise $\rho \in V^{\prime} \cap M P(N)$ so let $\left(M_{\rho}, s_{\rho}\right)$ be the associated marked hyperbolic 3-manifold. Note that by properties (1) and (3) of the neighborhood $V^{\prime}$ we can use $s_{\rho}: N \rightarrow M_{\rho}$ to mark $M_{\rho}$.

By property (4), the length of each $s_{\rho}\left(\gamma_{i}\right)^{*}$ will be short in $M_{\rho}$ so we can drill out $s_{\rho}(\gamma)^{*}=s_{\rho}\left(\gamma_{1}\right)^{*} \cup \cdots \cup s_{\rho}\left(\gamma_{d}\right)^{*}$ and get a hyperbolic manifold $\widehat{M}_{\rho}$. Let

$$
\psi_{\rho}: M_{\rho}-\mathbb{T}_{\epsilon_{0}}\left(s_{\rho}(\gamma)^{*}\right) \rightarrow \widehat{M}_{\rho}-\mathbb{T}_{\epsilon_{0}}(T)
$$

be the inverse of the map $\phi$ from the drilling theorem (Theorem 4.1). Let $\bar{M}_{\rho}$ be the cover of $\widehat{M}_{\rho}$ associated to $\left(\psi_{\rho} \circ s_{\rho}\right)_{*}\left(\pi_{1}(N)\right)$. Let $\bar{f}_{\rho}: N \rightarrow \bar{M}_{\rho}$ be the lift of $\psi_{\rho} \circ s_{\rho}: N \rightarrow \widehat{M}_{\rho}$. Note that $s_{\rho}(N)$ will be contained in $M_{\rho}-\mathbb{T}_{\epsilon_{0}}\left(s_{\rho}(\gamma)^{*}\right)$ by (3), so it makes sense to compose with $\psi_{\rho}$. In [20, Lemma 3.3], Bromberg shows that the representation $\left(\bar{f}_{\rho}\right)_{*}: \pi_{1}(N) \rightarrow \pi_{1}\left(\bar{M}_{\rho}\right) \subset \operatorname{PSL}(2, \mathbb{C})$ is in $A H(N, P)$.

Thus we can define $\xi$ by

$$
\xi(\rho)= \begin{cases}\left(\bar{f}_{\rho}\right)_{*} & \text { if } \rho \in M P(N), \\ \rho & \text { if } \rho \in \operatorname{MP}_{0}(N, P) .\end{cases}
$$

The following Lemma is a restatement of [20, Lemma 3.4].

Lemma 5.12 The map $\xi$ is continuous at all points in $V^{\prime} \cap M P_{0}(N, P)$. 
Since $\xi: V^{\prime} \rightarrow A H(N, P)$ is continuous at points in $V^{\prime} \cap M P_{0}(N, P)$, and $M P_{0}(N, P)$ is an open subset of $A H(N, P)$, we can restrict $\xi$ to a smaller neighborhood $\sigma_{0} \in$ $V \subset V^{\prime} \subset M P(N) \cup M P_{0}(N, P)$ so that its image is contained in $M P_{0}(N, P)$ [20, Corollary 3.5]. This allows us to use $\xi(\rho)$ as the first coordinate of $\Psi(\rho)$ in the definition of $\Psi$. We now consider the second coordinate $q(\rho)$.

If $\rho \in V \cap M P_{0}(N, P)$, then we set $q(\rho)=(\infty, \ldots, \infty)$. Otherwise, we consider the covering $\pi_{\rho}: \bar{M}_{\rho} \rightarrow \widehat{M}_{\rho}$ induced by the image of the injection $\left(\psi_{\rho} \circ s_{\rho}\right)_{*}: \pi_{1}(N) \rightarrow$ $\pi_{1}\left(\widehat{M}_{\rho}\right)$. The group $\pi_{1}\left(\widehat{M}_{\rho}\right)$ is obtained from $\pi_{1}\left(\bar{M}_{\rho}\right)$ by the same construction described in Section 5.2. That is, $\xi(\rho)=\left(\bar{M}_{\rho}, \bar{f}_{\rho}\right)$ corresponds to some representation $\sigma \in M P_{0}(N, P)$ and there is a unique $\left(w_{1}, \ldots, w_{d}\right)$ such that the extension $\sigma_{w_{1}, \ldots, w_{d}}\left(\pi_{1}(\hat{N})\right)=\pi_{1}\left(\widehat{M}_{\rho}\right)$. We define this to be $q(\rho)=\left(w_{1}, \ldots, w_{d}\right)$.

Equivalently, $w_{i}$ is defined so that if we conjugate $\left(\psi_{\rho} \circ s_{\rho}\right)_{*}$ so that $\gamma_{i}$ is mapped to

$$
\left(\begin{array}{ll}
1 & 2 \\
0 & 1
\end{array}\right)
$$

then the unique nontrivial element $\beta_{i} \in \pi_{1}\left(\partial U_{i}\right) \subset \pi_{1}\left(\widehat{M}_{\rho}\right)$ that bounds a disk in $M_{\rho}$ will be

$$
\left(\begin{array}{cc}
1 & w_{i} \\
0 & 1
\end{array}\right)
$$

Now that we have defined $q(\rho)$, we can define $\Psi: V \rightarrow M P_{0}(N, P) \times \widehat{\mathbb{C}}^{d}$ by

$$
\Psi(\rho)=(\xi(\rho), q(\rho))
$$

for any $\rho \in V$. Note that we have defined $q(\rho)$ so that $\Psi(\rho) \in \mathcal{A}$ for all $\rho \in V$. Unlike $\Phi$, we only show $\Psi$ is continuous for points on the boundary of $M P(N)$. Although Lemma 5.13 is nearly identical to of [20, Proposition 3.8], we include a proof since this is one of instances where we must keep track of multiple cusps and therefore our setup differs from Bromberg's.

Lemma 5.13 The map $\Psi$ is continuous on $V \cap M P_{0}(N, P)$.

Proof Lemma 5.12 shows that $\xi$ is continuous on $V \cap M P_{0}(N, P)$. Now consider a sequence $\rho_{i} \rightarrow \sigma$ where $\sigma \in M P_{0}(N, P)$. Since $\Psi(\sigma)=(\sigma, \infty, \ldots, \infty)$ and we know $\xi\left(\rho_{i}\right) \rightarrow \sigma$, it suffices to show that $q\left(\rho_{i}\right) \rightarrow(\infty, \ldots, \infty)$. If $\rho_{i} \in M P_{0}(N, P)$ then $q\left(\rho_{i}\right)=(\infty, \ldots, \infty)$ so assume that $\rho_{i} \in V \cap M P(N)$. We will use the notation $q\left(\rho_{i}\right)=\left(w_{1, i}, \ldots, w_{d, i}\right)$ and show $w_{j, i} \rightarrow \infty$ as $i \rightarrow \infty$ for $j=1, \ldots, d$.

Now for each $j=1, \ldots, d$, the length of $\rho_{i}\left(\gamma_{j}\right) \rightarrow 0$ as $i \rightarrow \infty$ because $\rho_{i}\left(\gamma_{j}\right)$ is converging to a parabolic and length is a continuous function with respect to the algebraic topology on the deformation space. Proposition 4.14 shows that the normalized 
length of the $j$-th meridian, $\beta_{j}$, goes to infinity as $i$ goes to infinity. The normalized length is given by

$$
\frac{\left|w_{j, i}\right|}{\sqrt{2 \operatorname{Im}\left(w_{j, i}\right)}}
$$

If $w_{j, i}$ does not go to $\infty$, then we must have $\operatorname{Im}\left(w_{j, i}\right) \rightarrow 0$ as $i \rightarrow \infty$. This cannot happen by Corollary 5.6.

It follows that $q\left(\rho_{i}\right) \rightarrow(\infty, \ldots, \infty)=q(\sigma)$ proving $q$ is continuous at any point $\sigma \in V \cap M P_{0}(N, P)$. Thus, $\Psi$ is continuous on $V \cap M P_{0}(N, P)$.

\subsection{Local homeomorphism}

Recall, in Section 5.5 we defined $\Phi$ on a subset $\mathcal{A}_{K} \subset \mathcal{A}$ and showed $\Phi$ is continuous. We now claim that there is some subset of $\mathcal{A}_{K}$ on which $\Phi$ is continuous and injective. See [20, Proposition 3.9].

Lemma 5.14 Let $\sigma_{0} \in M P_{0}(N, P)$. There is some neighborhood $U$ of $\left(\sigma_{0}, \infty, \ldots, \infty\right)$ in $\mathcal{A}$ such that $\left.\Psi \circ \Phi\right|_{U}=\mathrm{id}$. In particular, $\Phi$ is injective on $U$.

Proof Let $V$ be the neighborhood of $\sigma_{0}$ on which $\Psi$ was defined. By the continuity of $\Phi$, we can find a neighborhood $U^{\prime}$ so that $\Phi\left(U^{\prime}\right)$ is contained in $V$, and for any $\left(\sigma, w_{1}, \ldots, w_{d}\right) \in U^{\prime}, \sigma \in V$. We now consider $\left.\Psi \circ \Phi\right|_{U^{\prime}}$.

Let $\left(\sigma, w_{1}, \ldots, w_{d}\right) \in U^{\prime}$. If $\left(w_{1}, \ldots, w_{d}\right)=(\infty, \ldots, \infty)$ then $\Phi(\sigma, \infty, \ldots, \infty)=$ $\sigma$ and $\Psi(\sigma)=(\sigma, \infty, \ldots, \infty)$. If $\left(w_{1}, \ldots, w_{d}\right) \neq(\infty, \ldots, \infty)$ then $\sigma_{w_{1}, \ldots, w_{d}} \in$ $\operatorname{MP}(\widehat{N}, \widehat{P})$. Recall that the definition of $\Phi$ in this case was

$$
\Phi\left(\sigma, w_{1}, \ldots, w_{d}\right)=\left(M_{\sigma, w}, f_{\sigma, w}\right)
$$

where $M_{\sigma, w}$ was the filling of $\widehat{M}_{\sigma, w}=\mathbb{H}^{3} / \sigma_{w_{1}, \ldots, w_{d}}\left(\pi_{1}(\widehat{N})\right)$ and $f_{\sigma, w}$ is the composition $\phi_{\sigma, w} \circ \pi_{\sigma, w} \circ f_{\sigma}$. Since $\sigma \in V$, the marking $f_{\sigma}$ is homotopic to a local isometry $s_{\sigma}:\left(N, g_{\sigma}\right) \rightarrow M_{\sigma}$ such that $s_{\sigma}(N) \subset M_{\sigma}^{\geq \epsilon_{0}}$ (see the four properties of the neighborhood $V^{\prime}$ defined in Section 5.6). Thus we can redefine the marking $f_{\sigma, w}=\phi_{\sigma, w} \circ \pi_{\sigma, w} \circ s_{\sigma}$ without changing the definition of $\Phi(\sigma, w)$. Also recall that $\pi_{\sigma, w}$ is a covering map and therefore a local isometry, and

$$
\phi_{\sigma, w}: \widehat{M}_{\sigma, w}-\mathbb{T}_{\epsilon_{0}}(T) \rightarrow M_{\sigma, w}-\mathbb{T}_{\epsilon_{0}}(\gamma)
$$

is a bilipschitz diffeomorphism. (Recall that $\Phi$ was originally defined on $\mathcal{A}_{K}$ so that for any $(\sigma, w) \in \mathcal{A}_{K}, \phi_{\sigma, w}$ is a bilipschitz diffeomorphism on $\widehat{M}_{\sigma, w}-\mathbb{T}_{\epsilon_{3}}(T)$. By possibly making $U^{\prime}$ smaller, we can assume that $\phi_{\sigma, w}$ is bilipschitz on the $\widehat{M}_{\sigma, w}-\mathbb{T}_{\epsilon_{0}}(T)$.) 
Thus $f_{\sigma, w}=\phi_{\sigma, w} \circ \pi_{\sigma, w} \circ s_{\sigma}$ is smooth, and we let $g_{\sigma, w}^{\prime}$ be the pullback metric on $N$ via $f_{\sigma, w}: N \rightarrow M_{\sigma, w}$.

By the assumption that $\Phi\left(U^{\prime}\right) \subset V$, we can find a homotopic marking $s_{\sigma, w} \simeq$ $f_{\sigma, w}: N \rightarrow M_{\sigma, w}$ satisfying the properties (1)-(4) listed prior to the definition of $\Psi$. However, we need that $f_{\sigma, w}$ is homotopic to $s_{\sigma, w}$ in $M_{\sigma, w}-\gamma$ in order to have the drilling construction in $\Psi$ be the inverse to the filling construction in $\Phi$.

Let $W$ be a neighborhood of $g_{\sigma_{0}}$ (the pullback metric via $s_{\sigma_{0}}$ ) in $H^{-1}(V) \subset \mathcal{H}(N)$ such that Theorem 5.11 applies. We first claim there is some $U \subset U^{\prime} \subset \mathcal{A}$ such that if $(\sigma, w) \in U$, then $g_{\sigma, w}^{\prime} \in W$. There is some $J$ such that if $\phi_{\sigma, w}$ is a $J$-bilipschitz diffeomorphism and $\sigma$ is sufficiently close to $\sigma_{0}$, then $g_{\sigma, w}^{\prime} \in W$. So, let $U \subset U^{\prime}$ be a neighborhood of $\left(\sigma_{0}, \infty, \ldots, \infty\right)$ in $\mathcal{A}$ such that for all $\left(\sigma, w_{1}, \ldots, w_{d}\right) \in U, \sigma$ is sufficiently close to $\sigma_{0}$, and for all $i,\left|w_{i}\right|$ is large enough so that the filling map $\phi_{\sigma, w}$ is a $J$-bilipschitz diffeomorphism.

Next we claim that there is a metric $g_{\sigma, w} \in \varsigma(V) \subset W$ and a locally isometry $s_{\sigma, w}:\left(N, g_{\sigma, w}\right) \rightarrow M_{\sigma, w}$ such that $f_{\sigma, w}$ is homotopic to $s_{\sigma, w}$ as a map into $M_{\sigma, w}-\gamma$. This claim follows from product structure of $W$ described in Theorem 5.11. More precisely, let $N_{\text {th }}$ be a thickening of $N$. Then we can extend the local isometry $f_{\sigma, w}:\left(N, g_{\sigma, w}^{\prime}\right) \rightarrow M_{\sigma, w}$ to a local isometry $f_{\sigma, w, \mathrm{th}}:\left(N_{\mathrm{th}}, g_{\sigma, w, \mathrm{th}}^{\prime}\right) \rightarrow M_{\sigma, w}$, where $g_{\sigma, w \text {,th }}^{\prime}$ is a hyperbolic metric on $N_{\text {th }}$ that restricts to $g_{\sigma, w}^{\prime}$ on $N \subset N_{\text {th }}$. Then there exists a locally flat embedding $i: N \rightarrow N_{\text {th }}$ isotopic to the identity such that $s_{\sigma, w}=f_{\sigma, w, \mathrm{th}} \circ i$. Thus $s_{\sigma, w}$ and $f_{\sigma, w}$ are homotopic as maps inside $f_{\sigma, w, \mathrm{th}}\left(N_{\mathrm{th}}\right) \subset M_{\sigma}$. Since $f_{\sigma, w}(N) \subset M_{\sigma, w}-\mathbb{T}_{\epsilon_{0}}(\gamma)$, we can assume that the neighborhood $W$ in $\mathcal{H}(N)$ is small enough so that $f_{\sigma, w \text {,th }}\left(N_{\mathrm{th}}\right) \subset M_{\sigma, w}-\gamma$. Thus, $f_{\sigma, w}$ and $s_{\sigma, w}$ are homotopic in $M_{\sigma, w}-\gamma$.

It now follows from the definitions that

$$
\Psi\left(\Phi\left(\sigma, w_{1}, \ldots, w_{d}\right)\right)=\Psi\left(M_{\sigma, w}, f_{\sigma, w}\right)=\Psi\left(M_{\sigma, w}, s_{\sigma, w}\right)=\left(\sigma, w_{1}, \ldots, w_{d}\right) .
$$

Lemma 5.15 Let $\rho \in M P(N) \cup M P_{0}(N, P)$. If $\Phi \circ \Psi$ is defined at $\rho$ then $\Phi \circ \Psi(\rho)=\rho$.

Proof If $\rho \in V \cap M P_{0}(N, P)$ then clearly $\Psi(\rho)=(\rho, \infty, \ldots, \infty)$ and $\Phi(\Psi(\rho))=\rho$. If $\rho \in V \cap M P(N)$, then recall that we can choose the marking $s_{\rho}: N \rightarrow M_{\rho}$ and define $\widehat{M}_{\rho}$ to be the $\gamma$-drilling of $M_{\rho}$. Then we let $\bar{M}_{\rho}$ be the cover of $\widehat{M}_{\rho}$ associated to $\left(\psi_{\rho} \circ s_{\rho}\right)_{*}\left(\pi_{1}(N)\right)$. If $\Psi(\rho)=\left(\sigma, w_{1}, \ldots, w_{d}\right)$ then $\bar{M}_{\rho}=M_{\sigma}, \bar{f}_{\rho} \simeq f_{\sigma}, \widehat{M}_{\rho}=\widehat{M}_{\sigma, w}$, and $M_{\rho}=M_{\sigma, w}$. (To see why $\widehat{M}_{\rho}=\widehat{M}_{\sigma, w}$ see [20, Proposition 3.10 and Section 6]. It only follows from the definitions that $\widehat{M}_{\sigma, w}$ covers $\widehat{M}_{\rho}$; however, Bromberg proves this cover is trivial.) Thus

$$
\pi_{\sigma, w} \circ f_{\sigma} \simeq \pi_{\rho} \circ \bar{f}_{\rho}=\psi_{\rho} \circ s_{\rho}
$$


since $\overline{f_{\rho}}$ was the lift of $\psi_{\rho} \circ s_{\rho}$. But then

$$
f_{\sigma, w}=\phi_{\sigma, w} \circ \pi_{\sigma, w} \circ f_{\sigma} \simeq \phi_{\sigma, w} \circ \psi_{\rho} \circ s_{\rho}=\psi_{\rho}^{-1} \circ \psi_{\rho} \circ s_{\rho}=s_{\rho} .
$$

So when we apply $\Phi$ to $\left(\sigma, w_{1}, \ldots, w_{d}\right)$, we get $\left(M_{\sigma, w}, f_{\sigma, w}\right)=\left(M_{\rho}, s_{\rho}\right)=\rho$.

Theorem 5.16 Let $\sigma_{0} \in M P_{0}(N, P)$. The map $\Phi$ is a local homeomorphism from $\mathcal{A}_{K}$ to $M P(N) \cup M P_{0}(N, P)$ at $\left(\sigma_{0}, \infty, \ldots, \infty\right)$.

Proof It follows from Lemma 5.10 that $\Phi$ is continuous and from Lemma 5.14 that $\Phi$ is injective on some neighborhood $U$ of $\left(\sigma_{0}, \infty, \ldots, \infty\right)$.

Certainly $\Phi(U)$ contains $\sigma_{0}$. We claim that $\Phi(U)$ contains some neighborhood $V$ of $\sigma_{0}$ in $M P(N) \cup M P_{0}(N, P)$. Suppose no such neighborhood exists. Then we can find a nested sequence of neighborhoods $V_{i}$ whose intersection is $\sigma_{0}$ and a sequence $\rho_{i} \in V_{i}$ such that $\rho_{i} \notin \Phi(U)$. Since $\rho_{i} \rightarrow \sigma_{0}$, and Lemma 5.13 says that $\Psi$ is continuous at $\sigma_{0}$ we have $\Psi\left(\rho_{i}\right) \rightarrow \Psi\left(\sigma_{0}\right)=\left(\sigma_{0}, \infty, \ldots, \infty\right)$. It follows that $\Psi\left(\rho_{i}\right) \in U$ for all sufficiently large $i$; however, this contradicts Lemma 5.15 which says that $\Phi\left(\Psi\left(\rho_{i}\right)\right)=\rho_{i} \notin \Phi(U)$ for sufficiently large $i$.

Hence, there is some neighborhood $V$ of $\sigma_{0}$ contained in $\Phi(U)$. Since $\Phi$ is continuous, $\Phi^{-1}(V)$ is a neighborhood of $\left(\sigma_{0}, \infty, \ldots, \infty\right)$ in $\mathcal{A}$ such that $\left.\Phi\right|_{\Phi^{-1}(V)}: \Phi^{-1}(V) \rightarrow V$ is a continuous bijection. The inverse map is given by $\Psi$, which is continuous on $V \cap M P_{0}(N, P)$ by Lemma 5.13 and on $V \cap M P(N)$ by invariance of domain. Hence $\Phi$ is a local homeomorphism at $\sigma_{0}$.

Remark Since the point $\sigma_{0} \in M P_{0}(N, P)$ that we fixed in the beginning of Section 5.6 and used throughout Section 5.6 and Section 5.7 was arbitrary, we have actually shown that $\Phi$ is a local homeomorphism at any $\sigma \in M P_{0}(N, P)$.

\section{8 $M P(N) \cup M P_{0}(N, P)$ is not locally connected}

In Proposition 5.9, we saw that there was a point $\sigma_{0} \in M P_{0}(N, P)$ such that $\mathcal{A}$ is not locally connected at $\left(\sigma_{0}, \infty, \ldots, \infty\right)$. By Theorem 5.16, $\Phi$ is a local homeomorphism from $\mathcal{A}$ to $M P(N) \cup M P_{0}(N, P)$ at $\left(\sigma_{0}, \infty, \ldots, \infty\right)$. Hence, $M P(N) \cup M P_{0}(N, P)$ is not locally connected at $\Phi\left(\sigma_{0}, \infty, \ldots, \infty\right)=\sigma_{0} \in M P_{0}(N, P)$. Thus we have shown:

Theorem 5.17 There exists $\sigma_{0} \in M P_{0}(N, P)$ such that $M P(N) \cup M P_{0}(N, P)$ is not locally connected at $\sigma_{0}$. 
By the Density Theorem (Theorem 2.2), $A H(N)$ is the closure of $M P(N) \cup M P_{0}(N, P)$. Of course, it does not follow directly from this that $A H(N)$ is not locally connected at $\sigma_{0}$. In order to conclude anything about the closure, we need more quantitative control over the components of a neighborhood $U$ of $\left(\sigma_{0}, \infty, \ldots, \infty\right)$ in $\mathcal{A}$, and what happens to these components under the map $\Phi$. By Lemma 5.8, there is lower bound to the distance between some of the components of $U$. In the next Section, we will use the filling theorem to show that this implies $\overline{\Phi(U)}$ has infinitely many components.

\section{$6 A H(S \times I)$ is not locally connected}

In this section, we prove Theorem 1.1 by contradiction. If one assumes $A H(S \times I)$ is locally connected, then one may use the filling theorem (Theorem 1.2) and Lemma 5.8 to derive a contradiction. Recall that for a point $\left(\sigma, w_{1}, \ldots, w_{d}\right) \in \mathcal{A}$ at which $\Phi$ is defined, one obtains a hyperbolic manifold $M_{\sigma, w}$ by filling the $d$ cusps of $\widehat{M}_{\sigma, w}=$ $\mathbb{H}^{3} / \sigma_{w}\left(\pi_{1}(\hat{N})\right)$. The manifold $M_{\sigma, w}$ does not depend on the order in which the cusps are filled so we can fill the first cusp last. Let $M_{\sigma, w}^{\prime}$ denote the manifold with a single rank-2 cusp obtained by filling all but the first cusp of $\widehat{M}_{\sigma, w}$. Equivalently, $M_{\sigma, w}^{\prime}$ is the $\gamma_{1}$-drilling of $\Phi\left(\sigma, w_{1}, \ldots, w_{d}\right) \in M P(N)$. Eventually we will use the $w_{1}$ coordinate to estimate the complex length of $\gamma_{1}$ in $\Phi\left(\sigma, w_{1}, \ldots, w_{d}\right)$. As an intermediate step, Lemma 6.1 bounds the change in the geometry of the first cusp while we perform the other $d-1$ fillings.

Let $q_{1}$ be the first coordinate of the map $q$ in the definition of $\Psi$. That is,

$$
q_{1}: V \cap M P(N) \rightarrow \mathcal{T}\left(T^{2}\right)
$$

is defined so that if $\Psi(\eta)=\left(\sigma, w_{1}, \ldots, w_{d}\right)$, then $q_{1}(\eta)=w_{1}$. This is a Teichmüller parameter for the first cusp in $\widehat{M}_{\sigma, w}$ in the sense that $\sigma_{w}$ is conjugate to a representation that sends

$$
\gamma_{1} \text { to }\left(\begin{array}{ll}
1 & 2 \\
0 & 1
\end{array}\right)
$$

and the meridian

$$
\beta_{1} \text { of } \partial U_{1} \text { to } \quad\left(\begin{array}{cc}
1 & q_{1}(\eta) \\
0 & 1
\end{array}\right)
$$

Now define $r_{1}: V \cap M P(N) \rightarrow \mathcal{T}\left(T^{2}\right)$ so that $r_{1}(\eta)$ is the Teichmüller parameter of the cusp of $M_{\sigma, w}^{\prime}$. That is, after $d-1$ cusps have been filled, we can conjugate $\pi_{1}\left(M_{\sigma, w}^{\prime}\right)$ so that the remaining cusp is marked by

$$
\gamma_{1} \mapsto\left(\begin{array}{ll}
1 & 2 \\
0 & 1
\end{array}\right) \quad \text { and } \quad \beta_{1} \mapsto\left(\begin{array}{cc}
1 & r_{1}(\eta) \\
0 & 1
\end{array}\right)
$$


The drilling theorem can be used to show that $q_{1}$ and $r_{1}$ are close in the following sense.

Lemma 6.1 Let $c>0, \kappa>0$. There is some $l_{0}>0$ such that for any $\eta \in M P(N)$ with $\min \left\{\operatorname{Im}\left(q_{1}(\eta)\right), \operatorname{Im}\left(r_{1}(\eta)\right)\right\}<\kappa$ and

then

$$
\sum_{i=1}^{d} l\left(\eta\left(\gamma_{i}\right)\right)<l_{0}
$$

$$
\left|q_{1}(\eta)-r_{1}(\eta)\right|<\frac{c}{4}
$$

Proof For any $\eta \in M P(N)$, let $M_{\eta}=\mathbb{H}^{3} / \eta\left(\pi_{1}(N)\right)$, let $M_{\sigma, w}^{\prime}$ denote the $\gamma_{1}$-drilling of $M_{\eta}$, and let $\widehat{M}_{\sigma, w}$ denote the $\bigcup_{i=2}^{d} \gamma_{i}$-drilling of $M_{\sigma, w}^{\prime}$.

The drilling theorem says that there exists $l_{1}$ such that if the length of $l\left(\eta\left(\gamma_{1}\right)\right)<l_{1}$ then there is a 2-bilipschitz map

$$
M_{\eta}-\mathbb{T}_{\epsilon_{3}}\left(\gamma_{1}\right) \rightarrow M_{\sigma, w}^{\prime}-\mathbb{T}_{\epsilon_{3}}\left(T_{1}\right) .
$$

This implies the lengths of $\gamma_{2}, \ldots, \gamma_{d}$ do not double when we drill $\gamma_{1}$.

Choose some $\varepsilon>0$ such that $\varepsilon e^{\varepsilon}<c /(4 \kappa)$. There exists some $J>1$ such that if $X_{1}, X_{2}$ are two points in $\mathcal{T}\left(T^{2}\right)$ and $\phi: X_{1} \rightarrow X_{2}$ is a $J$-bilipschitz diffeomorphism, then $d_{\mathcal{T}\left(T^{2}\right)}\left(X_{1}, X_{2}\right)<\varepsilon$.

By the drilling theorem, there is some $l_{2}$ such that if $\sum_{i=2}^{d} l_{M_{\sigma, w}^{\prime}}\left(\gamma_{i}\right)<l_{2}$ then there exists a $J$-bilipschitz diffeomorphism

$$
\phi: M_{\sigma, w}^{\prime}-\bigcup_{i=2}^{d} \mathbb{T}_{\epsilon_{3}}\left(\gamma_{i}\right) \rightarrow \widehat{M}_{\sigma, w}-\bigcup_{i=2}^{d} \mathbb{T}_{\epsilon_{3}}\left(T_{i}\right) .
$$

Now choose any $0<l_{0}<\min \left\{l_{1}, l_{2} / 2\right\}$. If $\sum_{i=1}^{d} l\left(\eta\left(\gamma_{i}\right)\right)<l_{0}$ then $l\left(\eta\left(\gamma_{1}\right)\right)<l_{0}<l_{1}$. This implies the lengths of $\gamma_{2}, \ldots, \gamma_{d}$ do not double as we do the first drilling. Thus,

$$
\sum_{i=2}^{d} l_{M_{\sigma, w}^{\prime}}\left(\gamma_{i}\right)<\sum_{i=2}^{d} 2 l\left(\eta\left(\gamma_{i}\right)\right)<2 l_{0}<l_{2} .
$$

Now since $\sum_{i=2}^{d} l_{M_{\sigma, w}^{\prime}}\left(\gamma_{i}\right)<l_{2}$, there exists a $J$-bilipschitz diffeomorphism

$$
\phi: M_{\sigma, w}^{\prime}-\bigcup_{i=2}^{d} \mathbb{T}_{\epsilon_{3}}\left(\gamma_{i}\right) \rightarrow \widehat{M}_{\sigma, w}-\bigcup_{i=2}^{d} \mathbb{T}_{\epsilon_{3}}\left(T_{i}\right)
$$

when we drill $\gamma_{2}, \ldots, \gamma_{d}$. As in the proof of Corollary 4.13 (see also the remarks following Theorem 4.1), we can assume that $\phi$ restricts to a $J$-bilipschitz diffeomorphism on $T_{1}$ that takes torus cross-sections of the first cusp in $M_{\sigma, w}^{\prime}$ to torus cross-sections 
of the first cusp in $\widehat{M}_{\sigma, w}$ [11, Theorem 6.12]. Since the Teichmüller metric for $\mathcal{T}\left(T^{2}\right)$ agrees with the hyperbolic metric for the upper-half plane model of $\mathbb{H}^{2}$, this implies

$$
d_{\mathcal{T}\left(T^{2}\right)}\left(q_{1}(\eta), r_{1}(\eta)\right)=d_{\mathbb{H}^{2}}\left(q_{1}(\eta), r_{1}(\eta)\right)<\varepsilon
$$

See also [17, Theorem 7.2].

Since either $\operatorname{Im}\left(q_{1}(\eta)\right)<\kappa$ or $\operatorname{Im}\left(r_{1}(\eta)\right)<\kappa$,

$$
\left|q_{1}(\eta)-r_{1}(\eta)\right|<\kappa e^{\varepsilon}\left(d_{\mathbb{H}^{2}}\left(q_{1}(\eta), r_{1}(\eta)\right)\right)<\kappa \varepsilon e^{\varepsilon}<\frac{c}{4} .
$$

With Lemma 6.1 providing some control on $r_{1}$ based on $q_{1}$, we are now ready to prove Theorem 1.1, which we restate here for convenience.

Theorem 1.1 Let $S$ be a closed surface of genus $g \geq 2$. Then $A H(S \times I)$ is not locally connected.

Proof Let $\left(\sigma_{0}, \infty, \ldots, \infty\right) \in \mathcal{A}$ be the point that we described in Section 5.4 where we found $\mathcal{A}$ is not locally connected. Recall this was a point such that $\left.\sigma_{0}\right|_{\pi_{1}\left(N_{1,1}\right)}=\sigma_{z_{0}}$ where $z_{0}$ was the point described in Lemma 5.7. We will show $A H(S \times I)$ is not locally connected at $\sigma_{0}$. First we claim there exists a neighborhood $U$ of $\left(\sigma_{0}, \infty, \ldots, \infty\right)$ with the following properties:

(1) There is a neighborhood $V$ of $\sigma_{0}$ in $M P(N) \cup M P_{0}(N, P)$ such that $\left.\Phi\right|_{U}: U \rightarrow V$ is a homeomorphism. Such a neighborhood exists by Theorem 5.16.

(2) For any $\left(\sigma, w_{1}, \ldots, w_{d}\right) \in U,\left.\sigma\right|_{\pi_{1}\left(N_{1,1}\right)}$ lies in the neighborhood $W$ of $\left.\sigma_{0}\right|_{\pi_{1}\left(N_{1,1}\right)}$ that we defined in Section 5.4. Recall $W$ is a neighborhood of $\sigma_{z_{0}}$ in $M P_{0}\left(N_{1,1}, P_{1,1}^{\prime}\right)$ such that for all $\sigma_{z} \in \bar{W}$, the coordinate $z$ lies in the $\delta$-neighborhood $O$ of $z_{0}$. By Lemma 5.7, this means $\mathcal{A}_{z} \cap \operatorname{int}(R) \neq \varnothing$ and there is a constant $c>0$ such that the distance between $\overline{\mathcal{A}_{z}}$ and $\partial R$ is at least $c$.

(3) Recall from Section 5.4 that $C_{n}=\left\{\left(\sigma, w_{1}, \ldots, w_{d}\right) \in U: w_{1} \in R+2 n\right\}$. Then there exists $0<c<1$ such that for any $\left(\sigma, w_{1}, \ldots, w_{d}\right) \in \overline{C_{n}}$ and any $\left(\sigma^{\prime}, w_{1}^{\prime}, \ldots, w_{d}^{\prime}\right) \in$ $\overline{U-C_{n}}$, we have $\left|w_{1}-w_{1}^{\prime}\right|>c$ for any $n$. This follows from property (2) and Lemma 5.8.

(4) Let $c>0$ be the constant from (3). Let $\kappa>80(2 \pi)^{2}$ be some constant such that for any $\left(\sigma, w_{1}, \ldots, w_{d}\right) \in \overline{C_{n}}$, we have $\operatorname{Im}\left(w_{1}\right)<\kappa-1$. Then for any $\eta \in \Phi(U) \cap M P(N)=$ $V \cap M P(N)$, we have $\left|r_{1}(\eta)-q_{1}(\eta)\right|<c / 4$ or $\min \left\{\operatorname{Im}\left(r_{1}(\eta)\right), \operatorname{Im}\left(q_{1}(\eta)\right)\right\} \geq \kappa$.

We can assume $U$ satisfies property (4) for the following reason. First, the upper bound $\kappa$ on $\operatorname{Im}\left(w_{1}\right)$ exists because $w_{1}$ lies in the compact rectangle $R+2 n$. This was the 
definition of a point $\left(\sigma, w_{1}, \ldots, w_{d}\right)$ in $\overline{C_{n}}$. By Lemma 6.1 , given any $c, \kappa>0$ there exists some $l_{0}$ such that if $\sum_{i=1}^{d} l\left(\eta\left(\gamma_{i}\right)\right)<l_{0}$ then either $\left|r_{1}(\eta)-q_{1}(\eta)\right|<c / 4$ or $\min \left\{\operatorname{Im}\left(r_{1}(\eta)\right), \operatorname{Im}\left(q_{1}(\eta)\right)\right\} \geq \kappa$. Since $\left.\Phi\right|_{U}: U \rightarrow V$ is a homeomorphism, and $V$ is a neighborhood of $\sigma_{0}$ where $\sigma_{0}\left(\gamma_{1}\right), \ldots, \sigma_{0}\left(\gamma_{d}\right)$ are parabolic, we can make $U$ small enough so that $\sum_{i=1}^{d} l\left(\eta\left(\gamma_{i}\right)\right)<l_{0}$ for any $\eta \in \Phi(U) \cap M P(N)$. One can check that shrinking $U$ does not change properties (1), (2) and (3).

Again, since making $U$ smaller does not affect the above properties, we can assume for all $\left(\sigma, w_{1}, \ldots, w_{d}\right) \in U,\left|w_{1}\right|>81(2 \pi)^{2}$. Since $w_{1}=q_{1}\left(\Phi\left(\sigma, w_{1}, \ldots, w_{d}\right)\right)$, it follows from (3) and (4) that $U$ satisfies:

(5) For any $\left(\sigma, w_{1}, \ldots, w_{d}\right) \in U,\left|r_{1}\left(\Phi\left(\sigma, w_{1}, \ldots, w_{d}\right)\right)\right|>80(2 \pi)^{2}$.

Now that we have set up a neighborhood $U$ of $\left(\sigma_{0}, \infty, \ldots, \infty\right)$ in $\mathcal{A}$, suppose $A H(N)$ was locally connected at $\Phi\left(\sigma_{0}, \infty, \ldots, \infty\right)=\sigma_{0}$. Then we claim that, for all but finitely many $n$,

$$
\overline{\Phi\left(C_{n}\right)} \cap \overline{\Phi\left(U-C_{n}\right)} \neq \varnothing .
$$

To prove the claim, let $V_{A H}$ be a neighborhood (in $A H(N)$ ) of $\sigma_{0}$ contained inside $\overline{\Phi(U)}$. Note that the closure of $\Phi(U)$ contains such a neighborhood of $\sigma_{0}$ in $A H(N)$ by the Density Theorem (Theorem 2.2). If $A H(N)$ is locally connected, then there exists a connected neighborhood $\sigma_{0} \in V_{\text {conn }} \subset V_{A H}$. Recall in Section 5.4 we found a sequence $\left(\sigma_{0}, u_{1}+2 n, \ldots, u_{d}+2 n\right) \in C_{n}$ converging to $\left(\sigma_{0}, \infty, \ldots, \infty\right)$. Thus $V_{\text {conn }} \cap \Phi\left(C_{n}\right)$ and $V_{\text {conn }} \cap \Phi\left(U-C_{n}\right)$ are nonempty for all sufficiently large $n$.

If the closures of $\Phi\left(C_{n}\right)$ and $\Phi\left(U-C_{n}\right)$ were disjoint then we could form a separation of $V_{\text {conn }}$. Thus we must have

$$
\overline{\Phi\left(C_{n}\right)} \cap \overline{\Phi\left(U-C_{n}\right)} \neq \varnothing
$$

for all but finitely many $n$.

Now let

$$
\rho \in \overline{\Phi\left(C_{n}\right)} \cap \overline{\Phi\left(U-C_{n}\right)} .
$$

for some sufficiently large $n$. We will determine $n$ later, but for now assume that there are only finitely many $n$ for which this intersection is empty.

Although $\rho$ is not in the image of $\Phi$, we can find sequences

$$
\rho=\lim _{m \rightarrow \infty} \eta_{m}=\lim _{m \rightarrow \infty} \eta_{m}^{\prime},
$$

where $\eta_{m} \in \Phi\left(C_{n}\right)$ and $\eta_{m}^{\prime} \in \Phi\left(U-C_{n}\right)$ are representations in $M P(N)$. 
Up to subsequence, we can assume that $q_{1}\left(\eta_{m}\right)$ and $q_{1}\left(\eta_{m}^{\prime}\right)$ converge in $\widehat{\mathbb{C}}$, so we define $w_{1}$ and $w_{1}^{\prime}$ by

$$
\begin{aligned}
& w_{1}=\lim _{m \rightarrow \infty} q_{1}\left(\eta_{m}\right), \\
& w_{1}^{\prime}=\lim _{m \rightarrow \infty} q_{1}\left(\eta_{m}^{\prime}\right) .
\end{aligned}
$$

Equivalently, $w_{1}$ and $w_{1}^{\prime}$ are the second coordinates of $\lim _{m \rightarrow \infty} \Psi\left(\eta_{m}\right) \in \overline{C_{n}}$ and $\lim _{m \rightarrow \infty} \Psi\left(\eta_{m}^{\prime}\right) \in \overline{U-C_{n}}$. Note that $w_{1} \in R+2 n$ since $\eta_{m} \in \Phi\left(C_{n}\right)$ for all $m$. Thus $w_{1} \neq \infty$. Assume $w_{1}^{\prime} \neq \infty$ as well. We will discuss the possibility that $w_{1}^{\prime}=\infty$ below as a limiting case. Also, by passing to further subsequences if necessary, we define $\zeta_{1}$ and $\zeta_{1}^{\prime}$ by

$$
\begin{aligned}
& \zeta_{1}=\lim _{m \rightarrow \infty} r_{1}\left(\eta_{m}\right), \\
& \zeta_{1}^{\prime}=\lim _{m \rightarrow \infty} r_{1}\left(\eta_{m}^{\prime}\right) .
\end{aligned}
$$

Again, assume for now that $\zeta_{1}, \zeta_{1}^{\prime} \neq \infty$, and we will discuss the possibility that one or both of these sequences goes to infinity below.

By property (3) of $U$, there is some $0<c<1$ such that

$$
\left|w_{1}-w_{1}^{\prime}\right|>c .
$$

Note that $\kappa$ was chosen so that $\operatorname{Im}\left(w_{1}\right)<\kappa-1$ since $w_{1}=\lim q_{1}\left(\eta_{m}\right)$ and $\eta_{m} \in \Phi\left(C_{n}\right)$. Thus by property (4) of the neighborhood $U$, we have

$$
\left|\zeta_{1}-w_{1}\right| \leq \frac{c}{4}
$$

If we also have $\min \left\{\operatorname{Im}\left(w_{1}^{\prime}\right), \operatorname{Im}\left(\zeta_{1}^{\prime}\right)\right\}<\kappa$, then $\left|\zeta_{1}^{\prime}-w_{1}^{\prime}\right| \leq c / 4$ and thus

$$
\left|\zeta_{1}-\zeta_{1}^{\prime}\right| \geq c-\frac{c}{4}-\frac{c}{4}=\frac{c}{2} .
$$

Otherwise, we have $\min \left\{\operatorname{Im}\left(w_{1}^{\prime}\right), \operatorname{Im}\left(\zeta_{1}^{\prime}\right)\right\} \geq \kappa$. But since $\operatorname{Im}\left(w_{1}\right)<\kappa-1$ and $\left|w_{1}-\zeta_{1}\right| \leq c / 4$, we must have $\operatorname{Im}\left(\zeta_{1}\right)<\kappa-1+c / 4$. Thus

$$
\left|\zeta_{1}-\zeta_{1}^{\prime}\right| \geq\left|\operatorname{Im}\left(\zeta_{1}\right)-\operatorname{Im}\left(\zeta_{1}^{\prime}\right)\right|>\kappa-\left(\kappa-1+\frac{c}{4}\right)=1-\frac{c}{4}>\frac{c}{2}
$$

so inequality (18) still holds.

Next we will use the complex length estimates in the filling theorem to produce a contradiction to (18). Consider the complex length, $\mathcal{L}\left(\rho\left(\gamma_{1}\right)\right)$. We can estimate the complex length of $\rho\left(\gamma_{1}\right)$ in two ways, corresponding to each of the two sequences $\eta_{m}$ and $\eta_{m}^{\prime}$. 
For any $\eta \in V$, parts (ii) and (v) of the filling theorem (Theorem 1.2) can be used to estimate $\mathcal{L}\left(\eta\left(\gamma_{1}\right)\right)$. If we let

$$
L_{\eta}^{2}=\frac{\left|r_{1}(\eta)\right|^{2}}{2 \operatorname{Im}\left(r_{1}(\eta)\right)} \quad \text { and } \quad A_{\eta}^{2}=\frac{\left|r_{1}(\eta)\right|^{2}}{2 \operatorname{Re}\left(r_{1}(\eta)\right)}
$$

the filling theorem gives us the following estimates on $\mathcal{L}\left(\eta\left(\gamma_{1}\right)\right)=l\left(\eta\left(\gamma_{1}\right)\right)+i \theta\left(\eta\left(\gamma_{1}\right)\right)$ :

$$
\left|l\left(\eta\left(\gamma_{1}\right)\right)-\frac{2 \pi}{L_{\eta}^{2}}\right| \leq \frac{8(2 \pi)^{3}}{L_{\eta}^{4}-(16)(2 \pi)^{4}} \quad \text { and } \quad\left|\theta\left(\eta\left(\gamma_{1}\right)\right)-\frac{2 \pi}{A_{\eta}^{2}}\right| \leq \frac{5(2 \pi)^{3}}{\left(L_{\eta}^{2}-4(2 \pi)^{2}\right)^{2}} .
$$

If $\rho=\lim _{m \rightarrow \infty} \eta_{m}=\lim _{m \rightarrow \infty} \eta_{m}^{\prime}$, then we get the following two sets of estimates on $\mathcal{L}\left(\rho\left(\gamma_{1}\right)\right)=l\left(\rho\left(\gamma_{1}\right)\right)+i \theta\left(\rho\left(\gamma_{1}\right)\right)$. Let

$$
L^{2}=\lim _{m \rightarrow \infty} L_{\eta_{m}}^{2}, \quad A^{2}=\lim _{m \rightarrow \infty} A_{\eta_{m}}^{2}, \quad\left(L^{\prime}\right)^{2}=\lim _{m \rightarrow \infty} L_{\eta_{m}^{\prime}}^{2}, \quad\left(A^{\prime}\right)^{2}=\lim _{m \rightarrow \infty} A_{\eta_{m}^{\prime}}^{2} .
$$

Then

$$
\begin{gathered}
\left|l\left(\rho\left(\gamma_{1}\right)\right)-\frac{2 \pi}{L^{2}}\right| \leq \frac{8(2 \pi)^{3}}{L^{4}-(16)(2 \pi)^{4}}, \quad\left|\theta\left(\rho\left(\gamma_{1}\right)\right)-\frac{2 \pi}{A^{2}}\right| \leq \frac{5(2 \pi)^{3}}{\left(L^{2}-4(2 \pi)^{2}\right)^{2}}, \\
\left|l\left(\rho\left(\gamma_{1}\right)\right)-\frac{2 \pi}{\left(L^{\prime}\right)^{2}}\right| \leq \frac{8(2 \pi)^{3}}{\left(L^{\prime}\right)^{4}-(16)(2 \pi)^{4}},\left|\theta\left(\rho\left(\gamma_{1}\right)\right)-\frac{2 \pi}{\left(A^{\prime}\right)^{2}}\right| \leq \frac{5(2 \pi)^{3}}{\left(\left(L^{\prime}\right)^{2}-4(2 \pi)^{2}\right)^{2}} .
\end{gathered}
$$

Recall that by property (5) of the neighborhood $U$, we have $\left|r_{1}\left(\eta_{m}\right)\right|,\left|r_{1}\left(\eta_{m}^{\prime}\right)\right|>$ $80(2 \pi)^{2}$ for all $m$. So after passing to the limit, $\left|\zeta_{1}\right|,\left|\zeta_{1}^{\prime}\right| \geq 80(2 \pi)^{2}$ and $L^{2},\left(L^{\prime}\right)^{2} \geq$ $40(2 \pi)^{2}$. In particular, $L^{2},\left(L^{\prime}\right)^{2}>8(2 \pi)^{2}$, which together with the triangle inequality implies

$$
\begin{aligned}
& \left|\frac{2 \pi}{L^{2}}-\frac{2 \pi}{\left(L^{\prime}\right)^{2}}\right| \leq 16(2 \pi)^{3}\left(\frac{1}{L^{4}}+\frac{1}{\left(L^{\prime}\right)^{4}}\right), \\
& \left|\frac{2 \pi}{A^{2}}-\frac{2 \pi}{\left(A^{\prime}\right)^{2}}\right| \leq 20(2 \pi)^{3}\left(\frac{1}{L^{4}}+\frac{1}{\left(L^{\prime}\right)^{4}}\right) .
\end{aligned}
$$

Next, we combine the inequalities (19) and (20) to show that $\zeta_{1}$ and $\zeta_{1}^{\prime}$ are close. The following lemma provides a way of doing this. We refer the reader to [47, Lemma 6.2] for a detailed proof of this estimate, which is an intricate, but not deep, calculation.

Lemma 6.2 Let $z_{1}, z_{2} \in \mathbb{C},\left|z_{i}\right| \geq(80)(2 \pi)^{2}$, and set

$$
L_{i}^{2}=\frac{\left|z_{i}\right|^{2}}{2 \operatorname{Im}\left(z_{i}\right)} \text { and } A_{i}^{2}=\frac{\left|z_{i}\right|^{2}}{2 \operatorname{Re}\left(z_{i}\right)} .
$$


Suppose

$$
\begin{aligned}
& \left|\frac{2 \pi}{L_{1}^{2}}-\frac{2 \pi}{L_{2}^{2}}\right| \leq 16(2 \pi)^{3}\left(\frac{1}{L_{1}^{4}}+\frac{1}{L_{2}^{4}}\right), \\
& \left|\frac{2 \pi}{A_{1}^{2}}-\frac{2 \pi}{A_{2}^{2}}\right| \leq 20(2 \pi)^{3}\left(\frac{1}{L_{1}^{4}}+\frac{1}{L_{2}^{4}}\right) .
\end{aligned}
$$

Then

$$
\left|z_{1}-z_{2}\right|<560(2 \pi)^{2} \frac{\operatorname{Im}\left(z_{1}\right)}{\left|z_{1}\right|} .
$$

Setting $z_{1}=\zeta_{1}$ and $z_{2}=\zeta_{1}^{\prime}$, the inequalities (19) and (20), together with Lemma 6.2, imply

$$
\left|\zeta_{1}-\zeta_{1}^{\prime}\right|<560(2 \pi)^{2} \frac{\operatorname{Im}\left(\zeta_{1}\right)}{\left|\zeta_{1}\right|}
$$

At this point we address the possibility that $\zeta_{1}^{\prime}=\infty$. Notice that since $w_{1} \neq \infty$, the inequality $\left|w_{1}-\zeta_{1}\right| \leq c / 4$ implies $\zeta_{1} \neq \infty$. If $\zeta_{1}^{\prime}=\infty$ then inequality (21) is absurd since the right hand side is finite. Even though Lemma 6.2 only applies to points in $\mathbb{C}$, we can apply it to $z_{1}=\zeta_{1}$ and a sequence of points $z_{2}$ approaching $\infty$ to produce this contradiction. Hence $\zeta_{1}^{\prime}$ must be finite.

By combining the lower bound from (18) and the upper bound from (21), we find that

$$
\frac{c}{2}<560(2 \pi)^{2} \frac{\operatorname{Im}\left(\zeta_{1}\right)}{\left|\zeta_{1}\right|}
$$

Recall that the constant $\kappa$ was chosen in property (4) of $U$ so that $\operatorname{Im}\left(q_{1}\left(\eta_{m}\right)\right)<\kappa-1$ for any $\eta_{m} \in \Phi\left(C_{n}\right)$. Thus $\left|r_{1}\left(\eta_{m}\right)-q_{1}\left(\eta_{m}\right)\right|<c / 4$ for all $m$. It follows that $\operatorname{Im}\left(\zeta_{1}\right)$ is bounded above by a quantity that is independent of $n$ :

$$
\operatorname{Im}\left(\zeta_{1}\right) \leq(\kappa-1)+\frac{c}{4}<\kappa
$$

Since the Maskit slice $\mathcal{M}^{+}$is invariant under horizontal translations by 2 , we can assume for any point $w \in R, \operatorname{Re}(w)>-2$ (see Lemma 5.7). Since $\zeta_{1}$ lies in a closed (c/4)-neighborhood of $R+2 n$, we have $\left|\zeta_{1}\right| \geq 2 n-2-c / 4>2 n-3$. It follows that

$$
\frac{c}{2}<560(2 \pi)^{2} \frac{\operatorname{Im}\left(\zeta_{1}\right)}{\left|\zeta_{1}\right|}<\frac{560(2 \pi)^{2} \kappa}{2 n-3}
$$

Since $\kappa$ is a constant independent of $n$, there are only finitely many $n$ that satisfy (22). Hence, for any $\rho \in \overline{\Phi\left(C_{n}\right)} \cap \overline{\Phi\left(U-C_{n}\right)}$ with $n>560(2 \pi)^{2} \kappa / c+3 / 2$, inequality (22) produces a contradiction. However, our assumption that $A H(N)$ was locally connected implied that $\overline{\Phi\left(C_{n}\right)} \cap \overline{\Phi\left(U-C_{n}\right)}$ is nonempty for all but finitely many $n$.

It follows that $A H(N)$ is not locally connected at the point $\sigma_{0} \in M P_{0}(N, P)$. 


\section{References}

[1] W Abikoff, B Maskit, Geometric decompositions of Kleinian groups, Amer. J. Math. 99 (1977) 687-697 MR0480992

[2] I Agol, Tameness of hyperbolic 3-manifolds arXiv:math.GT/0405568

[3] L Ahlfors, L Bers, Riemann's mapping theorem for variable metrics, Ann. of Math. 72 (1960) 385-404 MR0115006

[4] J W Anderson, R D Canary, Algebraic limits of Kleinian groups which rearrange the pages of a book, Invent. Math. 126 (1996) 205-214 MR1411128

[5] J W Anderson, R D Canary, D McCullough, The topology of deformation spaces of Kleinian groups, Ann. of Math. 152 (2000) 693-741 MR1815699

[6] R Benedetti, C Petronio, Lectures on hyperbolic geometry, Universitext, Springer, Berlin (1992) MR1219310

[7] L Bers, Spaces of Riemann surfaces as bounded domains, Bull. Amer. Math. Soc. 66 (1960) 98-103 MR0111835

[8] L Bers, Spaces of Kleinian groups, from: "Several Complex Variables, I (Proc. Conf., Univ. of Maryland, College Park, MD, 1970)", (J Horváth, editor), Springer, Berlin (1970) 9-34 MR0271333

[9] B H Bowditch, Geometrical finiteness for hyperbolic groups, J. Funct. Anal. 113 (1993) 245-317 MR1218098

[10] J Brock, Boundaries of Teichmüller spaces and end-invariants for hyperbolic 3manifolds, Duke Math. J. 106 (2001) 527-552 MR1813235

[11] J Brock, K Bromberg, On the density of geometrically finite Kleinian groups, Acta Math. 192 (2004) 33-93 MR2079598

[12] J Brock, K Bromberg, R D Canary, Y N Minsky, Local topology in deformation spaces of hyperbolic 3-manifolds, Geom. Topol. 15 (2011) 1169-1224 MR2831259

[13] J Brock, K Bromberg, R Evans, J Souto, Tameness on the boundary and Ahlfors' measure conjecture, Publ. Math. Inst. Hautes Études Sci. (2003) 145-166 MR2031201

[14] J Brock, R D Canary, Y N Minsky, The classification of finitely generated Kleinian groups, in preparation

[15] J Brock, R D Canary, Y N Minsky, The classification of Kleinian surface groups, II: The ending lamination conjecture arXiv:math.GT/0412006

[16] K Bromberg, Rigidity of hyperbolic 3-manifolds with geometrically finite ends, $\mathrm{PhD}$ thesis, University of California, Berkeley (1998)

[17] K Bromberg, Hyperbolic cone-manifolds, short geodesics, and Schwarzian derivatives, J. Amer. Math. Soc. 17 (2004) 783-826 MR2083468 
[18] K Bromberg, Rigidity of geometrically finite hyperbolic cone-manifolds, Geom. Dedicata 105 (2004) 143-170 MR2057249

[19] K Bromberg, Projective structures with degenerate holonomy and the Bers density conjecture, Ann. of Math. 166 (2007) 77-93 MR2342691

[20] K Bromberg, The space of Kleinian punctured torus groups is not locally connected, Duke Math. J. 156 (2011) 387-427 MR2772066

[21] K Bromberg, J Holt, Self-bumping of deformation spaces of hyperbolic 3-manifolds, J. Differential Geom. 57 (2001) 47-65 MR1871491

[22] K Bromberg, J Souto, The density conjecture: A prehistoric approach, in preparation

[23] R Brooks, J P Matelski, Collars in Kleinian groups, Duke Math. J. 49 (1982) 163-182 MR650375

[24] D Calegari, D Gabai, Shrinkwrapping and the taming of hyperbolic 3-manifolds, J. Amer. Math. Soc. 19 (2006) 385-446 MR2188131

[25] R D Canary, Introductory bumponomics: the topology of deformation spaces of hyperbolic 3-manifolds, from: "Teichmüller theory and moduli problem", (I Biswas, R S Kulkarni, S Mitra, editors), Ramanujan Math. Soc. Lect. Notes Ser. 10, Ramanujan Math. Soc., Mysore (2010) 131-150 MR2667553

[26] R D Canary, D B A Epstein, P Green, Notes on notes of Thurston, from: "Analytical and geometric aspects of hyperbolic space (Coventry/Durham, 1984)", (D B A Epstein, editor), London Math. Soc. Lecture Note Ser. 111, Cambridge Univ. Press (1987) 3-92 MR903850

[27] R D Canary, D McCullough, Homotopy equivalences of 3-manifolds and deformation theory of Kleinian groups, 172, no. 812, Amer. Math. Soc. (2004) MR2096234

[28] V Chuckrow, On Schottky groups with applications to kleinian groups, Ann. of Math. 88 (1968) 47-61 MR0227403

[29] M Culler, P B Shalen, Varieties of group representations and splittings of 3-manifolds, Ann. of Math. 117 (1983) 109-146 MR683804

[30] A Douady, J H Hubbard, On the dynamics of polynomial-like mappings, Ann. Sci. École Norm. Sup. 18 (1985) 287-343 MR816367

[31] R Evans, J Holt, Non-wrapping of hyperbolic interval bundles, Geom. Funct. Anal. 18 (2008) 98-119 MR2399097

[32] W Heil, On the existence of incompressible surfaces in certain 3-manifolds. II, Proc. Amer. Math. soc. 25 (1970) 429-432 MR0266222

[33] C D Hodgson, S P Kerckhoff, Rigidity of hyperbolic cone-manifolds and hyperbolic Dehn surgery, J. Differential Geom. 48 (1998) 1-59 MR1622600

[34] C D Hodgson, S P Kerckhoff, Universal bounds for hyperbolic Dehn surgery, Ann. of Math. 162 (2005) 367-421 MR2178964 
[35] T Jørgensen, On discrete groups of Möbius transformations, Amer. J. Math. 98 (1976) 739-749 MR0427627

[36] M Kapovich, Hyperbolic manifolds and discrete groups, Progress in Math. 183, Birkhäuser, Boston (2001) MR1792613

[37] L Keen, C Series, Pleating coordinates for the Maskit embedding of the Teichmüller space of punctured tori, Topology 32 (1993) 719-749 MR1241870

[38] S P Kerckhoff, W P Thurston, Noncontinuity of the action of the modular group at Bers' boundary of Teichmüller space, Invent. Math. 100 (1990) 25-47 MR1037141

[39] I Kim, C Lecuire, K Ohshika, Convergence of freely decomposable Kleinian groups arXiv:0708.3266

[40] G Kleineidam, J Souto, Algebraic convergence of function groups, Comment. Math. Helv. 77 (2002) 244-269 MR1915041

[41] S Kojima, Deformations of hyperbolic 3-cone-manifolds, J. Differential Geom. 49 (1998) 469-516 MR1669649

[42] I Kra, On spaces of Kleinian groups, Comment. Math. Helv. 47 (1972) 53-69 MR0306485

[43] I Kra, Horocyclic coordinates for Riemann surfaces and moduli spaces. I: Teichmüller and Riemann spaces of Kleinian groups, J. Amer. Math. Soc. 3 (1990) 499-578 MR1049503

[44] R S Kulkarni, P B Shalen, On Ahlfors' finiteness theorem, Adv. Math. 76 (1989) 155-169 MR1013665

[45] P Lavaurs, Systèmes dynamiques holomorphes: Explosion de points périodique paraboliques, $\mathrm{PhD}$ thesis, Université Paris-Sud, Orsay (1989)

[46] C Lecuire, An extension of the Masur domain, from: "Spaces of Kleinian groups", London Math. Soc. Lecture Note Ser. 329, Cambridge Univ. Press (2006) 49-73 MR2258744

[47] A D Magid, Deformation spaces of Kleinian surface groups are not locally connected, PhD thesis, University of Michigan (2009) Available at http://www . math.umd.edu/ $\sim$ magid/papers.html

[48] A D Magid, Examples of relative deformation spaces that are not locally connected, Math. Ann. 344 (2009) 877-889 MR2507629

[49] A Marden, The geometry of finitely generated kleinian groups, Ann. of Math. 99 (1974) 383-462 MR0349992

[50] A Marden, Outer circles: An introduction to hyperbolic 3-manifolds, Cambridge Univ. Press (2007) MR2355387 
[51] B Maskit, Construction of Kleinian groups, from: "Proc. Conf. Complex Analysis (Minneapolis, 1964)”, (A Aeppli, E Calabi, H Röhrl, editors), Springer, Berlin (1965) 281-296 MR0178138

[52] B Maskit, On Klein's combination theorem, Trans. Amer. Math. Soc. 120 (1965) 499-509 MR0192047

[53] B Maskit, On Klein's combination theorem. II, Trans. Amer. Math. Soc. 131 (1968) 32-39 MR0223570

[54] B Maskit, Self-maps on Kleinian groups, Amer. J. Math. 93 (1971) 840-856 MR0291453

[55] D McCullough, Compact submanifolds of 3-manifolds with boundary, Quart. J. Math. Oxford Ser. 37 (1986) 299-307 MR854628

[56] C T McMullen, The classification of conformal dynamical systems, from: "Current developments in mathematics, 1995 (Cambridge, MA)”, (R Bott, M Hopkins, A Jaffe, I Singer, D Stroock, S-T Yau, editors), Int. Press, Cambridge, MA (1994) 323-360 MR1474980

[57] C T McMullen, Frontiers in complex dynamics, Bull. Amer. Math. Soc. 31 (1994) 155-172 MR1260523

[58] C T McMullen, Renormalization and 3-manifolds which fiber over the circle, Annals of Math. Studies 142, Princeton Univ. Press (1996) MR1401347

[59] C T McMullen, Complex earthquakes and Teichmüller theory, J. Amer. Math. Soc. 11 (1998) 283-320 MR1478844

[60] J Milnor, Remarks on iterated cubic maps, Experiment. Math. 1 (1992) 5-24 MR1181083

[61] Y N Minsky, The classification of punctured-torus groups, Ann. of Math. 149 (1999) 559-626 MR1689341

[62] J W Morgan, On Thurston's uniformization theorem for three-dimensional manifolds, from: "The Smith conjecture (New York, 1979)", (J W Morgan, H Bass, editors), Pure Appl. Math. 112, Academic Press, Orlando, FL (1984) 37-125 MR758464

[63] H Namazi, J Souto, Non-realizability and ending laminations, preprint (2010) Available at http://www.ma.utexas.edu/users/hossein/contents/ ending6.6.pdf

[64] K Ohshika, Ending laminations and boundaries for deformation spaces of Kleinian groups, J. London Math. Soc. 42 (1990) 111-121 MR1078179

[65] K Ohshika, Realising end invariants by limits of minimally parabolic, geometrically finite groups, Geom. Topol. 15 (2011) 827-890 MR2821565

[66] J S Purcell, Cusp shapes under cone deformation, J. Differential Geom. 80 (2008) 453-500 MR2472480 
[67] G P Scott, Finitely generated 3-manifold groups are finitely presented, J. London Math. Soc. 6 (1973) 437-440 MR0380763

[68] D Sullivan, Quasiconformal homeomorphisms and dynamics I. Solution of the FatouJulia problem on wandering domains, Ann. of Math. 122 (1985) 401-418 MR819553

[69] D Sullivan, Quasiconformal homeomorphisms and dynamics II. Structural stability implies hyperbolicity for Kleinian groups, Acta Math. 155 (1985) 243-260 MR806415

[70] W P Thurston, The geometry and topology of three-manifolds, Princeton Univ. Math. Dept. Lecture Notes (1979) Available at http://msri.org/publications/books/ gt $3 \mathrm{~m} /$

[71] W P Thurston, Three-dimensional manifolds, Kleinian groups and hyperbolic geometry, Bull. Amer. Math. Soc. 6 (1982) 357-381 MR648524

[72] W P Thurston, Hyperbolic structures on 3-manifolds I: Deformation of acylindrical manifolds, Ann. of Math. 124 (1986) 203-246 MR855294

[73] F Waldhausen, On irreducible 3-manifolds which are sufficiently large, Ann. of Math. 87 (1968) 56-88 MR0224099

[74] D J Wright, The shape of the boundary of Maskit's embedding of the Teichmüller space of once punctured tori, preprint (1987)

Department of Mathematics, University of Maryland

1301 Campus Drive, College Park MD 20742, USA

magid@math.umd.edu

Proposed: Danny Calegari

Seconded: David Gabai, Walter Neumann
Received: 23 March 2010

Revised: 19 January 2012 